\title{
Molecular Interactions between
}

\section{Neurons and Oligodendrocytes during Myelin Formation}

\author{
Dissertation \\ for the award of the degree \\ "Doctor rerum naturalium" \\ Division of Mathematics and Natural Sciences \\ of the Georg-August-Universität Göttingen
}

\author{
submitted by \\ Sebastian Timmler \\ born in Lutherstadt Wittenberg, Germany
}

Göttingen 2018 


\section{Members of the Thesis Committee:}

Prof. Dr. Mikael Simons, Reviewer

Max Planck Institute for Experimental Medicine, Göttingen

Institute of Neuronal Cell Biology, TU München

Prof. Dr. Blanche Schwappach, Reviewer

Institute for Molecular Biology (Universitätsmedizin Göttingen)

Georg-August-Universität Göttingen

Dr. Camin Dean

European Neuroscience Institute, Göttingen

Date of disputation: 09/17/2018, 1 pm at the lecture hall at the MPI of Experimental Medicine 


\section{Affidavit}

I solemnly declare that the present work was prepared independently and without using anything other than the named sources and aids. The texts, illustrations and/or ideas taken directly or indirectly from other sources (including electronic resources) have without exception been acknowledged and have been referenced in accordance with academic guidelines. The electronic version is identical to the printed document. The work or excerpts from it has not been submitted, in the same or a similar form, in satisfaction of the requirements of any other examination or at any other educational institution.

München, 2018 


\section{Contents}

Affidavit III

Contents IV

$\begin{array}{llll}\text { List of Figures } & \text { VIII }\end{array}$

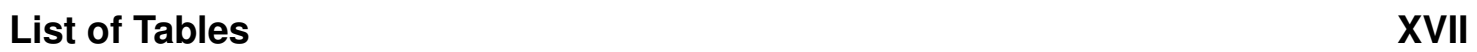

$\begin{array}{lll}\text { Abbreviations } & \text { XVIII }\end{array}$

$\begin{array}{ll}\text { Acknowledgments } & \text { XXI }\end{array}$

$\begin{array}{lll}\text { Abstract XXIII } & \text { X }\end{array}$

1 Introduction 1

1.1 The evolution of myelin . . . . . . . . . . . . . . . . . . 1

1.2 Myelin in the peripheral and central nervous system . . . . . . . . . . . . 7

1.3 Generation of CNS myelin . . . . . . . . . . . . . . 8

1.3.1 1) Initiation . . . . . . . . . . . . . . . . . . . . 10

1.3.2 2) Elongation and Compaction . . . . . . . . . . . . . . . . . 11

1.3.3 3) Termination . . . . . . . . . . . . . . . . . . 12

1.4 The structure of CNS myelin sheaths . . . . . . . . . . . . . . . . 14

1.4.1 The paranodal adhesion complex (CASPR/CNTN1/NFASC155) . . . 14

1.4.2 The juxtaparanodal adhesion complex (CASPR2/CNTN2/CNTN2) . . 17

1.4.3 Gangliosides and MAG interaction . . . . . . . . . . . . . . . . 18

1.5 Myelin and cellular adhesion molecules in diseases . . . . . . . . . . . . . 21

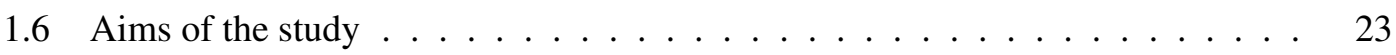


2 Materials and Methods $\quad 24$

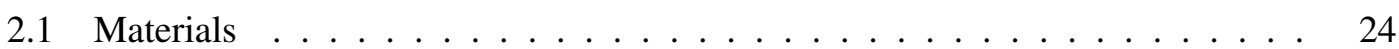

$2.1 .1 \quad$ Buffer conditions . . . . . . . . . . . . . . . . . . 24

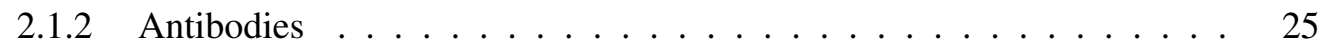

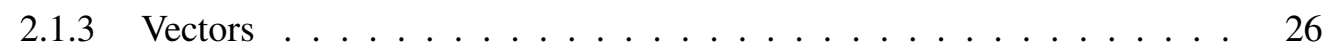

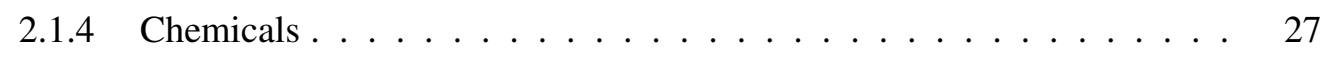

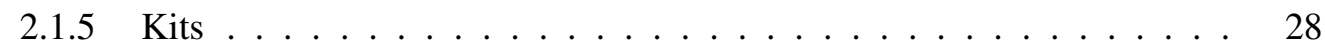

2.1 .6 Instruments . . . . . . . . . . . . . . . . . . . . . . . . 29

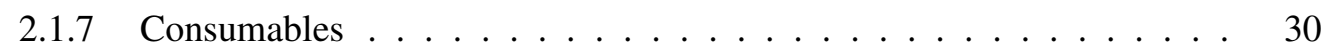

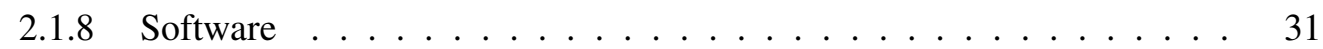

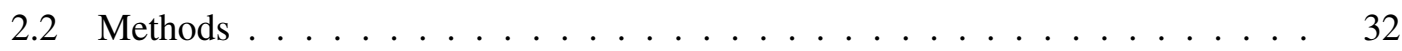

2.2 .1 Mouse husbandry . . . . . . . . . . . . . 32

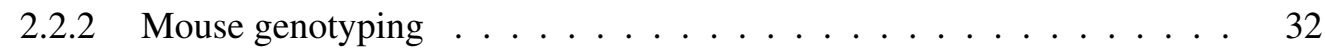

2.2.3 Immunohistochemistry of mouse tissue - conventional cryosectioning

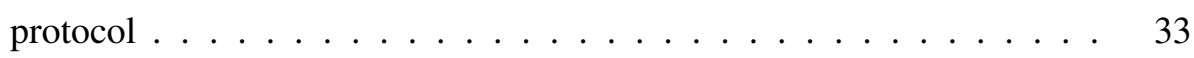

2.2.4 Immunohistochemistry of mouse tissue - vibratome, long incubation

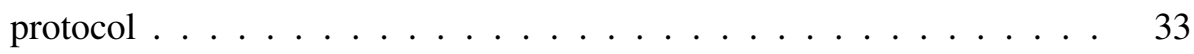

2.2.5 Expansion microscopy . . . . . . . . . . . . . . . . . . 34

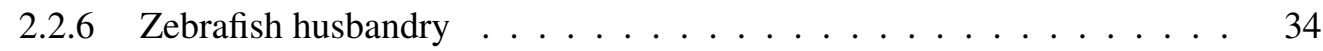

2.2 .7 Zebrafish genotyping . . . . . . . . . . . . . . 35

2.2 .8 Generation of mutant zebrafish . . . . . . . . . . . . . 35

2.2 .9 Imaging in zebrafish $\ldots \ldots \ldots \ldots$. . . . . . . . . . . 40

2.2.10 Electron Microscopy - conventional fixation of mouse tissue . . . . . . 41

2.2.11 Electron Microscopy - fixation of mouse tissue by high pressure freezing 42

2.2.12 Electron Microscopy - conventional fixation of fish tissue . . . . . . . . 43

2.2 .13 Electron Microscopy - sectioning $\ldots \ldots . \ldots 43$

2.2.14 Electron Microscopy - FIB-SEM . . . . . . . . . . . . . . . 44 
2.2.15 Electron Microscopy - ATUM . . . . . . . . . . . . . . . . 44

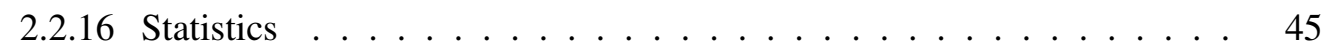

3 Results 46

3.1 Localization of the paranodal adhesion proteins in early development . . . . . . 46

3.1 .1 Mouse . . . . . . . . . . . . . . . . . . 46

3.1 .2 Fish . . . . . . . . . . . . . . . . . . 47

3.2 Single depletion of internodal and paranodal adhesion molecules . . . . . . . 48

3.2.1 Disruption of the paranodal adhesion complex leads to cell body myelination in zebrafish $\ldots \ldots \ldots \ldots \ldots \ldots$

3.2.2 Mag regulates lateral growth of zebrafish myelin sheaths . . . . . . . 49

3.2.3 Caspr-localization is not impaired in Mag-depleted fish or mice . . . . 52

3.3 Combined depletion of internodal and paranodal adhesion molecules causes defects in myelin lateral growth . . . . . . . . . . . . . . . 53

3.4 Mag together with the paranodal adhesion complex regulate targeting and wrapping of murine myelin sheaths _ . . . . . . . . . . . . . . . . . . 59

3.4.1 Conventional electron microscopy - mouse optic nerve cross sections . 59

3.4.2 Electron microscopy of high pressure frozen optic nerve longitudinal sections - Loss of the nodes of Ranvier _ . . . . . . . . . . . 65

3.4.3 3D reconstruction of optic nerve multiply myelinated axons $\ldots \ldots 74$

4 Discussion $\quad 79$

4.1 Localization of the paranodal junction complex $\ldots \ldots \ldots$

4.2 Paranodal junction proteins are required for correct myelin targeting . . . . . . 81

4.3 Mag is regulating lateral growth in zebrafish . . . . . . . . . 83 
4.4 Paranodal proteins and Mag synergisticly coordinate lateral extension and cor-

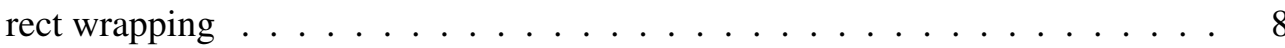

4.4.1 Reducing paranodal and internodal adhesion in developing myelin by

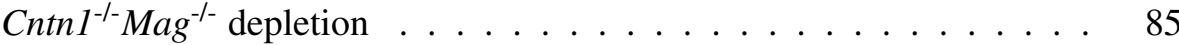

4.4.2 Reducing paranodal, juxtaparanodal and internodal adhesion in mature

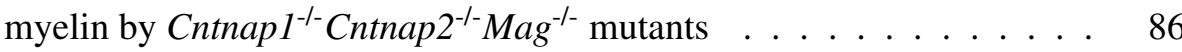

4.4.3 Two subtypes of split myelin are present in mutants lacking paranodal and internodal adhesion: doubled sheaths and "inside-out wrapping" . .

5 Conclusion

References 


\section{List of Figures}

1 Phylogeny of myelin and myelin-like structures. Underlinded: Taxa with myelin-like structures. red: vertebrate myelin. (modified, according to Hartline et al. [19]) . . . . . . . . . . . . . . . . .

2 Model of CNS myelin sheath development (a-d) The model indicates the movement of the inner tongue and parallel compaction. The geometry of the membrane and the closing of cytoplasmic channels are demonstrated in the unwrapped representation. Figure from Snaidero et al. (Cell 2014), Elsevier, License number: 4393570735342 [82] . . . . . . . . . . . . . . . . . . . . 13

3 Molecular structure at the axon-oligodendroglial interface Mag is located at the internode, forms a dimer and binds to the gangliosides GT1a and GD1b on the neuronal side. Potassium channels (Kv1) are clustered at the juxtaparanodes by a complex of glial Cntn2 and neuronal Caspr2 and Cntn2. The paranodes are fixed by a complex of glial Nfasc155 and neuronal Caspr and Cntn1. Sodium channels (Nav1)are clustered at the nodes of Ranvier, together with Nfasc186. .

4 Stages of node development indicated by Caspr and AnkG stainings Representative images and measured spectra (a) paranodal [P] (b) paranodal+nodal $[\mathrm{PN}](\mathrm{c})$ paranodal+nodal peak [PNP](d) nodal [N], scalebars $=10 \mu \mathrm{m}$ (e) Stages of node development at P4, P7, P12 and P21 (f) nodal and paranodal distance in spinal cord sections at P4, P7, P12 and P21 (g) Caspr staining in paranodes, aquired using expansion microscopy, scale can not be set due to unknown ex-

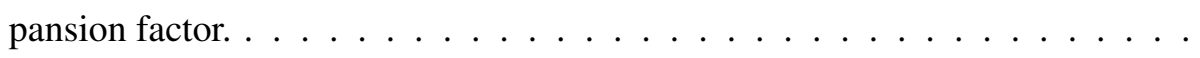


5 Myelinated cell bodies in paranodal protein mutant fish. (a) Micrographs of wildtype controls and mutant fish generated in $\operatorname{Tg}(\mathrm{mbp}$ :eGFP-CAAX), scale bar $=20 \mu \mathrm{m}$ (b) Quantification of myelinated cell bodies normalized to the myelinated area at 3, 4 and $10 \mathrm{dpf}$ (c) Quantification of myelinated cell bodies in wildtype animals at 3 and $4 \mathrm{dpf}$ (d) Quantification of retractions from cell bodies during 14 hrs, acquired by live imaging (e) Representative micrograph of a myelinated cell body with transient expression of mbp:mcherry in $\mathrm{Tg}$ (mbp:eGFP-CAAX) contactin 1 associated protein (cntnap1)--myelin associated glycoprotein $\left(\mathrm{mag}^{-{ }^{--}}\right.$, it proofs that circular profiles are neuronal cell bodies, scalebar $=20 \mu \mathrm{m}$; in all quantifications we performed ANOVA, followed by pairwise $\mathrm{t}$-test with $\mathrm{p}$-value adjustment by Bonferroni method. . . . . . . . .

6 Myelin properties in single and double mutant fish. (a) Micrographs of wildtype controls and all mutant fish generated in $\operatorname{Tg}(\mathrm{mbp}$ :eGFP_CAAX), scale bar $=50 \mu \mathrm{m}$ (b) Quantification of myelinated area from $10 \mathrm{dpf}$ dorsal spinal cord maximum projections, statistical test: Kruskal-Wallis, p-value adjustment by Dunn's method (c) Quantification of oligodendrocyte numbers in 4 dpf dorsal spinal cord (d) Representative micrographs of individual oligodendrocytes in dorsal spinal cord, scalebar $=25 \mu \mathrm{m}$ (e) Quantification of sheaths per oligodendrocyte from $3 \mathrm{dpf}$ dorsal spinal cord, ANOVA, followed by pairwise t-test, p-value adjustment by Bonferroni method (f) Quantification of myelin sheath length in $4 \mathrm{dpf}$ dorsal spinal cord ( $\mathrm{g}$ ) Quantification of myelin sheath length in 10 dpf dorsal spinal cord (h) Representative transmission electron microscopy (TEM) micrographs of fish spinal cord at $10 \mathrm{dpf}$, scale bar = $500 \mathrm{~nm}$ (i) Quantification of myelinated axons in TEM micrographs; in all quantifications we performed ANOVA, followed by pairwise t-test with p-value adjustment by Bonferroni method . . . . . . . . . . . . . . . . . . . . 5 
7 Transient expression of UAS:contactin 1 associated protein (caspr)-yellow fluorescent protein (YFP) under control of huc:Gal4 in Tg(mbp:eGFP_CAAX) mag $^{-/-}$fish (a) Micrographs of caspr localization, pink lines indicate where the spectrum was measured (average of 10 parallel lines), scalebar $=5 \mu \mathrm{m}$ (b) Intensity spectra along the respective line, black lines indicate the measured distance (c) Quantification of individual paranodal lengths in two fish per genotype

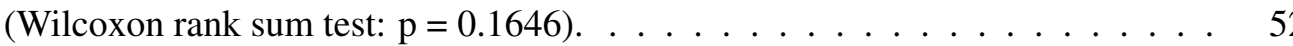

8 Immunohistochemistry approach to assess Contactin 1 associated protein (Caspr) localization in Myelin associated glycoprotein (Mag) deficient mice (a) Micrographs of wildtype and $\mathrm{Mag}^{-/-}$mice, pink lines indicate where the spectrum was measured (average of 10 parallel lines), scalebar $=\mu \mathrm{m}$ (b) Intensity spectra along the respective line, black lines indicate the measured distance (c) Quantification of individual paranodal lengths in two mice per genotype (Wilcoxon rank sum test: $\mathrm{p}=0.05469) \ldots \ldots \ldots \ldots \ldots$

9 Extension of myelin sheaths in wildtype and mutant fish (a) Sequential frames of a live imaging experiment in wildtype and contactinl $b(\text { cntn } 1 b)^{-/-} \mathrm{mag}^{-{ }_{-1}^{-}} \mathrm{mu}-$ tant, blue arrowheads indicating extending segments, orange arrowheads indicating slowly extending segments, scalebar $=5 \mu \mathrm{m}$ (b) Extension of single myelin sheaths during time in wildtyp animals, red indicates the fastest, blue the slowest sheath (c) Extension of single myelin sheaths during time in cntnap $^{-/-}$animals, red indicates the fastest, blue the slowest sheath (d) Extension of single myelin sheaths during time in $\mathrm{mag}^{-/-}$animals, red indicates the fastest, blue the slowest sheath. (e) Extension of single myelin sheaths during time in cntnap $^{-{ }^{-/}} \mathrm{mag}^{-/-}$ animals, red indicates the fastest, blue the slowest sheath. . . . . . . . . . 55 
10 Retraction of myelin sheaths in wildtype and mutant fish (a) Sequential frames of a live imaging experiment in wildtype and cntnap $^{-{ }_{-}}$mag $^{-/-}$mutant, blue arrowheads indicating retracting segments, orange arrowheads indicating retracting segments that start to disintegrate and blebb, scalebar $=5 \mu \mathrm{m}(\mathrm{b}) \mathrm{Re}-$ traction of single myelin sheaths during time in wildtyp animals, red indicates the fastest, blue the slowest sheath (c) Retraction of single myelin sheaths during time in cntnap $^{-{ }^{--}}$mutants, red indicates the fastest, blue the slowest sheath (d) Retraction of single myelin sheaths during time in $\mathrm{mag}^{-/-}$mutants, red indicates the fastest, blue the slowest sheath (e) Retraction of single myelin sheaths during time in cntnap $^{-{ }_{-}}$mag $^{-/-}$mutants, red indicates the fastest, blue the slowest

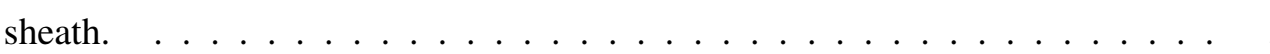

11 Quantification of extension and retraction rates (a) Average extension rates of single and double mutants (b) Average retraction rates of single and double mutants; in all quantifications we performed ANOVA, followed by pairwise ttest with p-value adjustment by Bonferroni method.

12 Blebbing of mutant myelin sheaths (a) Micrographs of a sheath disintegration event (blebbing) indicated by blue arrowheads, scalebar $=10 \mu \mathrm{m}$ (b) Quantification of coordinated retractions during $12 \mathrm{~h}$ (c) Quantification of blebbing events occured during $12 \mathrm{~h}$; in all quantifications we performed ANOVA, followed by pairwise t-test with p-value adjustment by Bonferroni method. 
13 Doubled layered myelin in fish (a) Confocal micrographs of overlapping myelin sheaths, demonstrated by expression of mbp:mcherry in $\operatorname{Tg}(\mathrm{mbp}$ :eGFP-CAAX fish), scale bar $=20 \mu \mathrm{m}$ (b) Quantification of double myelin across mutants, identified by $100 \%$ intensity step (c) TEM micrograph of an overgrown node in a longitudinal section of cntnap $^{-{ }^{-/}}$mag $^{-/-}$mutant fish spinal cord, scale bar = $500 \mathrm{~nm}$ (d) TEM micrograph of a node of Ranvier in a longitudinal section of wildtype fish spinal cord, scale bar $=500 \mathrm{~nm}$ (e) TEM micrograph of distorted loop organization in a longitudinal section of cntnapl $^{-{ }_{-}} \mathrm{mag}^{-/-}$mutant fish spinal cord, scale bar $=500 \mathrm{~nm} \ldots \ldots \ldots \ldots \ldots \ldots$

14 Properties of myelin in mouse mutants (a) TEM micrographs of optic nerve cross sections and quantification of myelinated axons at P12, statistical test: pairwise student's t-test, p-value adjustment by Bonferroni method (b) TEM micrographs of optic nerve cross sections and quantification of myelinated axons at P15, statistical test: pairwise student's t-test, p-value adjustment by Bonferroni method (c) Quantification of optic nerve g-ratio to diameter plot of Contactin1 $(\text { Cntnl })^{-/-} \mathrm{Mag}^{-/-}$mice and wildtype controls at P15 (d) Quantification of average g-ratio of both groups, statistical test: student's t-test (e) g-ratio histogram for both datasets (f) Representative confocal micrographs used to measure sheath lengths (g) Quantification of myelin sheath lengths in P12 single and double mutants, pairwise Wilcoxon rank sum test, p-value adjustment by Bonferroni method. 
15 Myelin defects present in $\mathrm{CntnI}^{-/-} \mathbf{M a g}^{-/-}$double mutants (a) Representative TEM micrographs of myelinated axons, degenerated myelin (whorls), split myelin and outfoldings (tomaculi) at P15 (b) Quantification of myelin defects at P12 in $\%$ of all myelinated axons observed in an individual (c) Quantification of myelin defects at P15 in \% of all myelinated axons observed in an individual; in all quantifications we performed ANOVA, followed by pairwise t-test with p-value adjustment by Bonferroni method. . . . . . . . . . . . . . .

16 Weight of mutant mice (a) Images of representative mouse mutants and wildtype mouse at P21 (b) Quantification of weight at P12/13 (c) Quantification of weight at P21/22; in all quantifications we performed ANOVA, followed by pairwise t-test with p-value adjustment by Bonferroni method.

17 Analysis of myelination and g-ratios in mice (a) Relative quantification of myelinated and unmyelinated axons (b) Quantification of average g-ratio of mutant mice at P21/22 (c) g-ratio to caliber distribution for wildtype and Cnt-

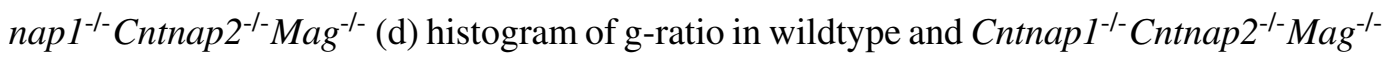
animals, analysis of 5 frames $\left(219 \mu \mathrm{m}^{2}\right)$ per animal, $\mathrm{n}=4$; in all quantifications we performed ANOVA, followed by pairwise t-test with p-value adjustment by Bonferroni method.

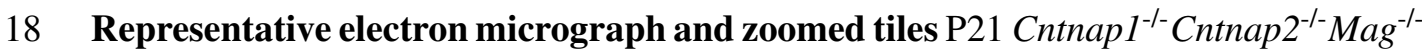
conventional fixed optic nerve cross section, demonstrating multiply myelinated axons and outfoldings, scalebar $=2000 \mu \mathrm{m}$.

19 Representative electron micrographs of (a) P12/13 optic nerve cross sections,

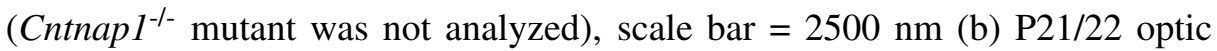
nerve cross sections, scale bar $=2500 \mathrm{~nm}$. 
20 Quantification of single and split myelinated axons in mice (a) Quantification of myelinated axons at P12/13 (b) Quantification of myelinated axons at P21/22 (c) Quantification of P12/13 split-myelinated axons P12/13 (d) Quantification of myelinated axons at $\mathrm{P} 21 / 22$, analysis of 10 frames $\left(219 \mu \mathrm{m}^{2}\right)$ per animal; in all quantifications we performed ANOVA, followed by pairwise t-test with p-value adjustment by Bonferroni method.

21 Electron micrographs of P21 single mutant and wildtype mice optic nerves, high pressure freezing fixation, showing nodes of Ranvier with paranodal loops, scalebar $=2 \mu \mathrm{m}$.

22 Electron micrographs of P21 double and triple mutant mice optic nerves, high pressure freezing fixation, showing nodes of Ranvier with paranodal loops and paranodal loop like structures, scalebar $=2 \mu \mathrm{m} \ldots \ldots \ldots \ldots$

23 Model, representative image and quantification of (a) [I] normal nodes (b) [II] solitary sheath ends (c) [III] loops under myelin (d) [IV] loops on myelin, scalebar $=1000 \mathrm{~nm}$; pairwise t-test, $\mathrm{p}$-value adjustment by Bonferroni method.

$24 \mathbf{N a}_{\mathbf{v}} 1.6$ staining in wildtype and mutant animals (a) Representative confocal micrographs showing $\mathrm{Na}_{\mathrm{v}} 1.6$ positive signals in mutant and wildtype optic nerve longitudinal sections, scale bar $=50 \mu \mathrm{m}$ (b) Quantification of nodes of Ranvier; ANOVA followed by pairwise t-test, p-value adjustment by Bonferroni method.

25 automated serial sections to tape (ATUM) micrograph of a P15 $\mathrm{CntnI}^{-/-} \mathrm{Mag}^{-/-}$ double mutant optic nerve (a) Representative micrographs of one ATUM section (b) Representative images of myelinated axons that were followed through the volume, to categorize in: inside-out myelin, double myelin or ambiguous situation. Colors correspond to the respective categories, scale bar $=2000 \mathrm{~nm}$. (Images were kindly provided by Dr. Martina Schifferer and Dr. Minou Djan-

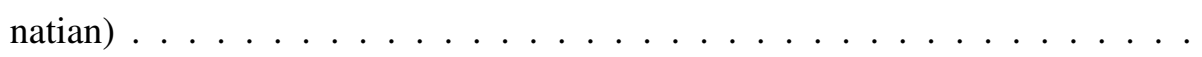


26 3D Serial block-face imaging by focused-ion beam milling in the scanning electron microscope (FIB-SEM) reconstruction of a P15 $\mathrm{CntnI}^{-/-} \mathrm{Mag}^{-/-}$double mutant optic nerve (a) Representative sections of the reconstructed axon (b) Reconstruction of an axon of the inside-out wrapping type, blue $=$ axon, green $=$ inner tongue, magenta $=$ secondary inner tonge, scale bar $=5 \mu \mathrm{m}$ (Images were kindly provided by Dr. Martina Schifferer, Dr. Minou Djannatian and Manja Luckner). . . . . . . . . . . . . . . . . .

27 3D FIB-SEM reconstruction of a P21 Cntnap $1^{-/-}$Cntnap2 $^{-/-}$Mag $^{-/-}$double mutant optic nerve (a) Representative sections of the reconstructed axon (b) Reconstruction of an axon of the inside-out wrapping type, blue $=$ axon, green $=$ inner tongue, magenta $=$ secondary inner tongue, scale bar $=5 \mu \mathrm{m} \ldots \ldots \ldots$

28 Model of overlapping myelin, presented in half cut view. (a) Model of wildtype node development (b) Model of overgrowing nodes and myelin double sheaths in mutants with reduced adhesion. One sheath is growing under its

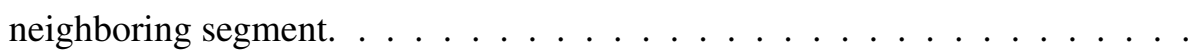

29 Model of "inside-out wrapping" in Contactin 1 associated protein (Cntnap1) $)^{-/-}$Mag $^{-/-}$ and $\mathrm{CntnI}^{-/-} \mathrm{Mag}^{-/-}$myelin. In wildtype myelin sheaths, the active inner tongue adds layers of membrane from the inside. The first wrap becomes the outermost paranodal loop. [I] When axon-glia-adhesion is reduced, the layers can be retracted, releasing the inner tongue from the center. [II] The inner tongue is able to slip over the edge of a retracted layer. [III] The inner tongue is pushing in between two layers. [IV] The inner tongue is split. The inner tongue is actively myelinating at the inside, the second inner tongue extends into the myelin and wraps in the opposite direction. 
30 Knock out validation by mRNA levels in Crispr/Cas9 edited fish, measured

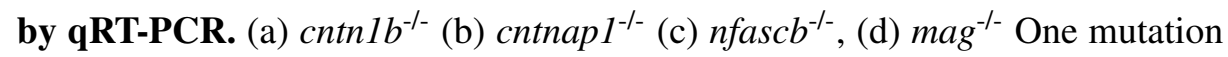
was tested for $c n t n 1 b, c n t n a p l, n f a s c b$ and two for mag. mRNA levels of $c n t n 1 b$ and $n f a s c b$ were reduced by about $40 \%$, mag and cntnapl mutants showed reduction by about $85 \%$

31 Confocal micrographs of the localization of paranodal proteins using fusion proteins (a) cntn $1 \mathrm{~b}$-mcherry at $4 \mathrm{dpf}$, scale bar $=3 \mu \mathrm{m}$ (b) cntn $1 \mathrm{~b}$-mcherry at $10 \mathrm{dpf}$, scale bar $=3 \mu \mathrm{m}$ (c) nfascb-mcherry at $3 \mathrm{dpf}$, scale bar $=5 \mu \mathrm{m}$ (d) caspr-YFP at 3dpf, scale bar $=5 \mu \mathrm{m} \ldots \ldots \ldots \ldots \ldots \ldots$ 


\section{List of Tables}

1 Myelin related proteins in fish, mice and humans $\ldots \ldots \ldots 6$

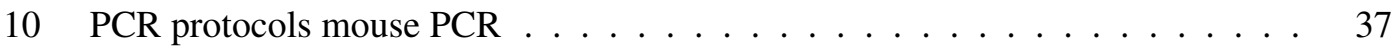

11 PCR protocols fish PCR . . . . . . . . . . . . . . . . . 37

$12 \quad$ PCR mix mouse genotyping $\ldots \ldots \ldots \ldots \ldots \ldots$

$13 \quad$ PCR $\operatorname{mix}$ fish genotyping $\ldots \ldots \ldots \ldots \ldots \ldots$

14 PCR digestion . . . . . . . . . . . . . . . . . . . . . 38

15 Mouse genotyping primer . . . . . . . . . . . . . . . . . . . . . 39

16 Zebrafish genotyping primer $\ldots \ldots \ldots \ldots \ldots$

17 gRNAs used to generate zebrafish mutant lines $\ldots \ldots \ldots$

18 Tissue Processing Protocol . . . . . . . . . . . . . . . . . . . . . 42 


\section{Abbreviations}

aa amino acid

AMC Arthrogryposis Multiplex Congenita

AnkG ankyrin G

ATUM automated serial sections to tape

caspr contactin 1 associated protein

Caspr Contactin 1 associated protein

CASPR Contactin 1 associated protein

Caspr2 Contactin 2 associated protein

CASPR2 Contactin 2 associated protein

cDNA complementary deoxynucleic acid

CGT ceramide galactosyltransferase

CHN Congenital Hypomyelinating Neuropathy

CNP 2'-3'-cyclic nucleotide-3'-phosphodiesterase

Cntn1 Contactin1

CNTN1 Contactin1

cntn1a contactin1 a

cntn1b contactin $1 \mathrm{~b}$

Cntn2 Contactin2

cntnap1 contactin 1 associated protein

Cntnap1 Contactin 1 associated protein

CNTNAP1 Contactin 1 associated protein

Cntnap2 Contactin 2 associated protein

CNTNAP2 Contactin 2 associated protein

CNS central nervous system

Crispr/Cas9 Clustered regularly interspaced short palindromic repeats/CRISPR-associated 9

DNA deoxynucleic acid

dpf days post fertilization

ECM extra cellular matrix

EGF epidermal growth factor

eGFP enhanced green fluorescent protein

EPON Epoxy resin

ER endoplasmatic reticulum

FIB-SEM Serial block-face imaging by focused-ion beam milling in the scanning electron microscope 


\begin{tabular}{|c|c|}
\hline Fyn & Fyn kinase \\
\hline GABA & gamma-aminobutyric acid \\
\hline Galgt1 & Beta-1,4 N-acetylgalactosaminyltransferase 1 \\
\hline GPI & glycosylphosphatidylinositol \\
\hline gRNA & guide ribonucleic acid \\
\hline Ig & Immunoglobulin \\
\hline Jam2 & Junctional adhesion molecule B \\
\hline $\mathbf{L 1}$ & Neural cell adhesion molecule L1 \\
\hline LCM & Lethal Congenital Myopathy \\
\hline mag & myelin associated glycoprotein \\
\hline Mag & Myelin associated glycoprotein \\
\hline MAG & Myelin Associated Glycoprotein \\
\hline Map2 & microtubuli associated protein 2 \\
\hline Mbp & Myelin basic protein \\
\hline MBP & Myelin basic protein \\
\hline MIB & microscopy imaging browser \\
\hline MRI & magnetic resonance imaging \\
\hline mRNA & Messenger ribonucleic acid \\
\hline MS & multiple sclerosis \\
\hline N-cam & Neural cell adhesion molecule \\
\hline Necl1 & Nectin-like 1 \\
\hline Necl4 & Nectin-like 4 \\
\hline NeuN & neuron-specific nuclear protein \\
\hline NF & neurofilament \\
\hline Nfasc & Neurofascin \\
\hline NFASC & Neurofascin \\
\hline nfasca & neurofascin a \\
\hline nfascb & neurofascin $b$ \\
\hline NMJ & neuro muscular junction \\
\hline nt & nucleotides \\
\hline OCT & optimal cutting temperature compound \\
\hline OPCs & oligodendrocyte precursor cells \\
\hline PAX2 & paired box protein 2 \\
\hline PBS & phosphate buffered saline \\
\hline PBS-GT & PBS with gelatine and triton- $x-100$ \\
\hline PFA & paraformaldehyde \\
\hline
\end{tabular}




$\begin{array}{ll}\text { PCR } & \text { polymerase chain reaction } \\ \text { Plp } & \text { Proteolipid-protein } \\ \text { PLP } & \text { Proteolipid-protein } \\ \text { PNS } & \text { peripheral nervous system } \\ \text { Pten } & \text { phosphatase and tensin homolog } \\ \text { PTU } & \text { 1-phenyl-2-thiourea } \\ \text { qRT-PCR } & \text { quantitative real-time polymerase chain reaction } \\ \text { Siglecs } & \text { sialic-acid-binding lectins } \\ \text { TAE } & \text { Tris-Acetat-EDTA buffer } \\ \text { TE } & \text { Tris-EDTA buffer } \\ \text { TEM } & \text { transmission electron microscopy } \\ \text { YFP } & \text { yellow fluorescent protein }\end{array}$




\section{Acknowledgment}

I would like to express my gratitude to my supervisor Prof. Dr. Mikael Simons for his patient guidance, support and helpful advice throughout this study. It is great to work in such an effective scientific environment.

I want to thank Dr. Minou Djannatian for excellent and intense co-working throughout the last year. She generated the cntn $1^{-{ }_{-}}$and $\mathrm{mag}^{-{ }^{-}}$fish and kindly provided data of $\mathrm{Cntn} 1^{1_{-}}$and $\mathrm{Cntn}^{-/-} \mathrm{Mag}^{-/-}$fish and mice used in this study.

I wish to thank the members of my thesis committee, Prof. Dr. Blanche Schwappach and Dr. Camin Dean, for challenging my ideas and giving inputs from a different perspective.

The people of the EM-facility at the MPI for Experimental Medicine in Göttingen helped me a lot developing my skills in electron microscopy from the scratch. I want to thank head of the facility, Dr. Wiebke Möbius for excellent guidance, Marie-Theres Weil for performing freeze-substitution and FIB-SEM on Cntnap $1^{-/-} \mathrm{Cntnap}^{2-\mathrm{Mag}^{-/-}}$mutants and Torben Ruhwedel for providing unlimited advice and conducting high-pressure freezing together with me.

EM samples of fish and $\mathrm{Cntn}^{-{ }^{--}}$and $\mathrm{Cntn} 1^{--_{-}} \mathrm{Mag}^{-{ }^{-}}$mutant mice were processed and acquired at the Synergy-EM facility in München. I want to thank head of the facility Dr. Martina Schifferer for her favorable support and the ATUM processing and Manja Luckner for the FIB-SEM experiment and reconstruction of the $\mathrm{Cntn}^{-{ }^{--}} \mathrm{Mag}^{-/-}$mutant.

I thank Martina Arends, who designed the Caspr-YFP construct, the gRNA for Caspr depletion and injected the founder generation as part of her master thesis.

Elior Peles and Hauke Werner provided mouse mutants to the project, thanks for that. I thank Tim Czopka, who provided many reporter constructs used in this study.

I want to express my gratitude to all animal care takers in the mouse facilities in Göttingen (Jennifer Schindler, Stefanie Thiel and Nadine Jagaschewskie) and München (Claudia Ihbe, Georg Esser and Stefanie Wurster) and the fish facility in München (Sabine Schlink and Roberto Rojas Rojas). I thank Ursula Fünfschilling, Anne von Thaden and Bettina Schmid for their various helpful advices on laboratory animals. 
I specially thank Ioannis Alexopoulos for his great ideas and fruitful discussions about image acquisition and data analysis.

The GGNB was always supportive and efficient, I want to thank the GGNB-team and the head of the CMPB program Prof. Dr. Michael Hörner.

My thanks go to all the other members of the AG Simons (past and present) and technical staff at both institutes, that I had a great time working with:

Mar Bosch-Queralt, Ludovico Cantuti-Castelvetri, Maria Ines Cunha, Dirk Fitzner, Karla Hans, Brigitte Haslbeck, Tina Kling, Maryam Khojastehfard, Marcel Matt, Chaitali Mukherjee, Liliana Pedro, Horst Penkert, Shima Safaiyan, Aldijana Salovic, Giselheid Schulz, Nicola Schwedhelm, Paula Sanchez, Minhui Su, Caroline Velte, Ulrich Weikert.

I want to express my gratitude to my parents that raised me up with patience, waking up my interest in science. They are great role-models providing me with inspiration and encouragement.

Franziska Barlage was constantly supporting me throughout the years and especially the last months. It is great to share day by day with you and having you in my life!

I thank all the friends from different places as Halle, Kiel, Göttingen and München. Your guys were a great support! 


\section{Abstract}

Myelin is a multi-layered membrane wrapped around axons. It allows fast nerve conduction and provides the axon with trophic support. In the central nervous system (CNS), oligodendrocytes form multiple myelin sheaths. Thus, a single oligodendrocyte has to coordinate the generation of numerous myelin segments on different axons. A cellular model of myelin sheath generation has been provided, but the molecular mechanisms of multiple aspects of myelination such as initiation, lateral growth and termination are still elusive. During myelination, the inner leaflet is actively growing by adding new membrane layers to the sheath. At the same time, the sheath is growing laterally and the outer layers are starting to compact. The adhesion molecules forming the paranodal Caspr/Cntn1/Nfasc 155 complex are essential for the formation of the Nodes of Ranvier (gaps inbetween individual sheaths), but whether they might play a role in guiding myelin growth and wrapping is not known.

In this study, we combined high-resolution live imaging with newest ultra-structure resolving 3D electron microscopy techniques to assess the role of the mayor axonglial adhesion factors. We sequentially reduced internodal (Mag) and paranodal (Caspr/Cntn1/Nfasc155) adhesion in zebrafish by Crispr/Cas9 gene editing and in mice by crossbreeding of established mutant lines. Zebrafish mutants exhibited myelinated cell bodies, decreased sheath length, impaired sheath growth dynamics and double myelinated sheaths. Consistent with the zebrafish data, double mutants in mice showed hypomyelination, reduced sheath length and split myelin sheaths. The split myelin sheaths could be identified as either overgrown double myelin segments or aberrant wrapping by a divided, misguided inner tongue, that we call "inside-out-wrapping". In our model we propose, that axon-oligodendroglial adhesion is essential for lateral growth, precise wrapping and correct targeting of the myelin sheath. This results do not only provide new insights into myelin biology, but may also have implications for our understanding of myelin formation in neurological diseases. 


\section{Introduction}

\subsection{The evolution of myelin}

\section{Two strategies for fast nerve conduction}

Life or death of living organisms depends on the speed of how information is transmitted in the nervous system. Skillfull predation and fast escape are the keys to evolutionary success: The Kangaroo rat Dipodomys deserti evades rattle snake attacks by jumps and kicks within $50 \mathrm{~ms}$ of reaction time [1]. When threatened, flying squids jump out of the water, covering a distance of several tens of meters [2] and the Peregrine Falcon (Falco peregrinus) attacks its prey at a speed of up to $320 \mathrm{~km} / \mathrm{h}$, making it the fastest animal on earth [3]. Fast perception and processing of information from the environment are essential for this astonishing survival strategies. Animals transfer information along specialized structures: the axons of nerve cells. Hence, nerve cell conduction velocity is directly affecting an organism's evolutionary success and selection will push the increase of neuronal speed to its physical limits. High nerve conduction velocity allows an individual to interact with its environment with minimal delay. Thus, the high complexity of the mammalian and even human behavior could not be reached without a fast calculating hardware working with nearly real-time precision.

Two electrical properties are essential to increase the speed of signal conduction along an axon: axial resistance of the axonal fiber and capacitance between the intra-axonal cytoplasma and the extra-axonal matrix. Axial resistance is dependent of the axon caliber. Therefore, thick axons transmit signals faster than thin ones in proportion to the square root of the inner diameter [4]. This relatively simple approach to increase conduction speed is reflected by the often seen phenomenon of axonal gigantism. In many species, especially invertebrates, neurons that require rapid conduction show an enormous diameter. Examples are the giant squid axon, nerves involved in tail-flip responses of lobsters and the paired giant motor neurons in annelids like Branchiomma [5][6]. Even very small animals that live on a very different time scale like cope pods and fruit flies developed giant axons [7]. 


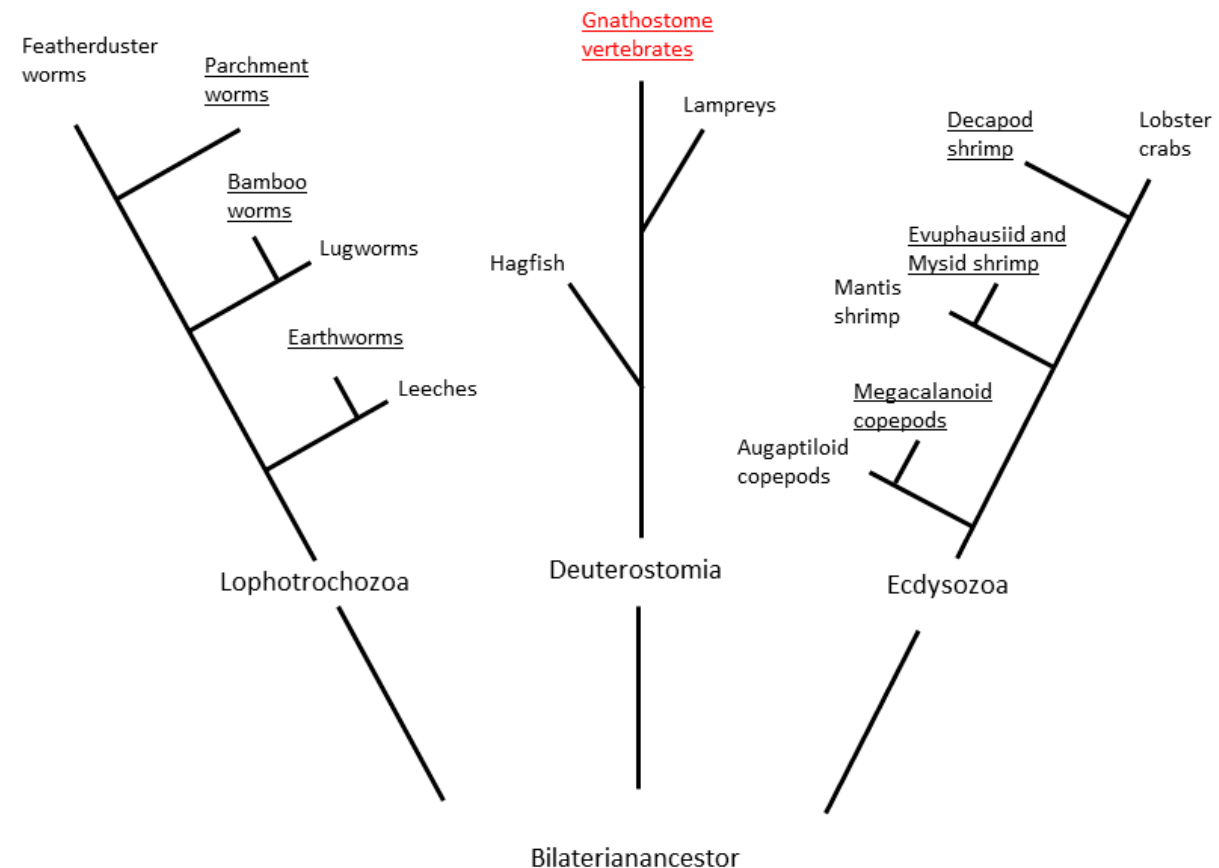

Figure 1: Phylogeny of myelin and myelin-like structures. Underlinded: Taxa with myelin-like structures. red: vertebrate myelin. (modified, according to Hartline et al. [19])

However, increasing the conduction speed through the capacitance challenged the evolution to establish a whole new way of cell-cell interaction using adhesion and strict control of electrical signals - myelin. Myelin or myelin-like structures appeared at least four times during evolution (twice in crustaceans, once in annelids, and once in gnathostomes) but always share the same feature: a lipid-rich multilammelar membrane that covers the axon (see Fig.1) [8][9]. In vertebrates, sheaths mainly consist of a helically wound glial membrane embedding the axon interspersed by gaps the so called 'nodes of Ranvier' [10]. Narrowing down the exact ecological conditions that led to myelin is difficult, but in some species, lifestyle and myelin occurrence can be correlated. Benthic and deep-sea cope pod species lack myelin in contrast to pelagic species that are exposed to visual predators, which is also reflected in different escape reaction times 
[11]. The kuruma shrimp (Penaeus japonicus) has axons with myelin-like structures that reach a conduction speed of about $200 \mathrm{~m} / \mathrm{s}$ and therefore are the fastest observed so far [12].

\section{Vertebrate myelin is unique}

Compared to a myelinated mammalian nerve, an unmyelinated squid axon of similar conduction needs 15.000 times the space and several thousand folds more energy. Under this conditions, a human spinal cord would have a diameter of about 1 meter [13]. Skull and spine limit the space and force the neurons to rely on a different mechanism of fast signal conduction. Therefore, nearly all known vertebrates have myelinated axons. First evidence for vertebrate myelin were found in placoderms, together with another evolutionary invention - the hinged jaw. Placodermi is an extinct class of fish, that lived in between 420 to 360 million years ago. Their heads were covered in armor plates and reached lengths of 7 to $9 \mathrm{~m} \mathrm{[13].} \mathrm{Fossils} \mathrm{of} \mathrm{this} \mathrm{prehistoric} \mathrm{fish}$ show imprints of cranial nerves that together with foramina in the skull can be used to estimate nerve diameter and length. Comparisons of the occulomotor nerve of placoderms and the older non-jaw osteostraci cladd showed that it had a similar width $(0.1 \mathrm{~mm})$ but was 10 -times longer in the placoderms [14]. Since the hinged-jawed fish were predators, it is very likely that they had myelinated axons - in contrast to the osteostraci with a passive lifestyle.

Vertebrate myelin reduces electrical capacitance and increases resistance between interior and exterior of the fiber through multilamellar lipid membranes. To keep up this function, current leakage must be blocked by tight adhesion at paranodes to prevent short circuits. In vertebrate and annelid myelin, the membranes are spirally wrapped, whereas electrically conductive cytoplasm and extra-cytoplasmic space are reduced by compaction of the myelin membranes, thereby blocking a possible current short cut through them. In vertebrates, internal and external leaflets of the lipid bi-layer are in close contact and form the distinctive alternating pattern of mayor dense line (intracellular) and intraperiod line (extracellular). In contrast to that, myelin of other species like the earthworm (Lumbricus terrestris L.) is compacted in some regions but not in others [15]. Neurons and glia evolved together long before one started to wrap the other. This led to strong interactions and dependencies, which is reflected in many studies showing 
trophic support of glia towards neurons [16]. Non-vertebrate myelin-like structures can lead to new insights on basic neuron-glia interactions, but studies on mechanisms of myelination require vertebrate model organisms like rat, mouse or zebrafish.

Myelinated axons provide high speed nerve conduction at a minimal space, allowing complex circuits and structures like the human brain. But this advantage comes at a price: Highly ordered and specialized structures are more susceptible to diseases. This is reflected in the considerable amount of myelin-related disorders that cause great personal suffering and high social costs [17].

\section{The zebrafish - a model organism for myelination}

The zebrafish Danio rerio is a tropical freshwater cyprinide that originates from the rice fields of India and Myanmar. Its main advantages are the small size $(\sim 3 \mathrm{~cm})$, short generation time ( $\sim 3$ months) and a weekly production of hundreds of eggs per female. Zebrafish were hold in captivity for a long time and discovered as a laboratory animal in the late 1960s by George Streisinger. In 1981, Streisinger cloned the first homozygous diploid zebrafish and by that opened up tremendous scientific opportunities [18]. Since the eggs can be observed from fertilization via embryogenesis to hatching and beyond, the zebrafish is an established model organism for research on development and diseases [20]. Fish can be made transparent by 1-phenyl2-thiourea (PTU)-treatment or genetic approaches which makes them suitable for all kinds of in vivo light microscopy, including live imaging techniques [21]. With recent advances in the Crispr/Cas9 technology and thanks to the development of the Tol2-kit, genetical modification of zebrafish embryos is relatively simple [22]. Transient knock outs or fluorescent reporters are easy to produce and generating stable knock out and reporter lines has become rather a matter of months than years [23]. The genome of the zebrafish is completely sequenced and accessible in databases. Compared to mammalia, the teleosts (fish) underwent whole genome duplication after seperation from the common ancestor. During evolution, most gene duplicates were inactivated (non-functionalization) and some remained the same to keep the dosage balance (dosage selection). On the other hand, some gene copies split up the tasks (subfunctionalization) and some duplications were free to invent new functions (neofunctionalization) [24]. For translation 
of results to mammals or even humans, it is therefore very important to check for gene duplications of orthologs and be aware of possible differences. In the case of this myelin-related study, Mag and Cntnap1 have no paralog, whereas Cntn1 and Neurofascin (Nfasc) appear as the genetic duplicates $a$ and $b$ [25]. Zebrafish and mammalian myelin is very similar in cellular and molecular structure and most of the human genes have closely related zebrafish orthologs. Due to the genome duplication, some human splice variants of one gene are split up to independent genes in fish (e.g. Proteolipid-protein (PLP) and DM20). In terms of fish myelin, the most prominent difference is the use of P0 protein instead of PLP as the major central nervous system (CNS) myelin protein component [26]. Zebrafish and mammal P0 sequence is widely conserved, but the $\mathrm{P} 0$ promotor region of the fish is closer related to the mammal promoter region of PLP than P0. Experiments with enhanced green fluorescent protein (eGFP) expressed in zebrafish under the control of the mouse PLP-promoter show oligodendroglial localization [27]. That leads to the conclusion, that zebrafish oligodendrocytes and Schwann cells are not as clearly distinguishable by biochemichal features as in mammals [28]. However, the myelin structure and cell lineage relationships are highly conserved [29][30]. Orthologues for all major myelin related proteins can be identified in zebrafish.(See Tab1) Expression pattern and localization during time are mostly similar to mammals. One special evolutionary feature of fish is the pairwise, ventrally located Mauthner axon, which is fully myelinated without nodes of Ranvier. The two Mauthner axons span the whole spinal cord and process fast escape reflexes. Mauthner myelin sheaths have 200-350 lammella and grow throughout life-time [31]. The signal is still transferred by saltatory nerve conduction with axon collaterals taking over the function of the nodes [32][33]. Mauthner axons show that there is more than one way to transmit action potentials and to form myelin, but they were not part of this study due to lack of representation in mammals. Beside of the Mauthner axon, the general mechanisms of myelination are comparable between fish and mammals [34][35]. 


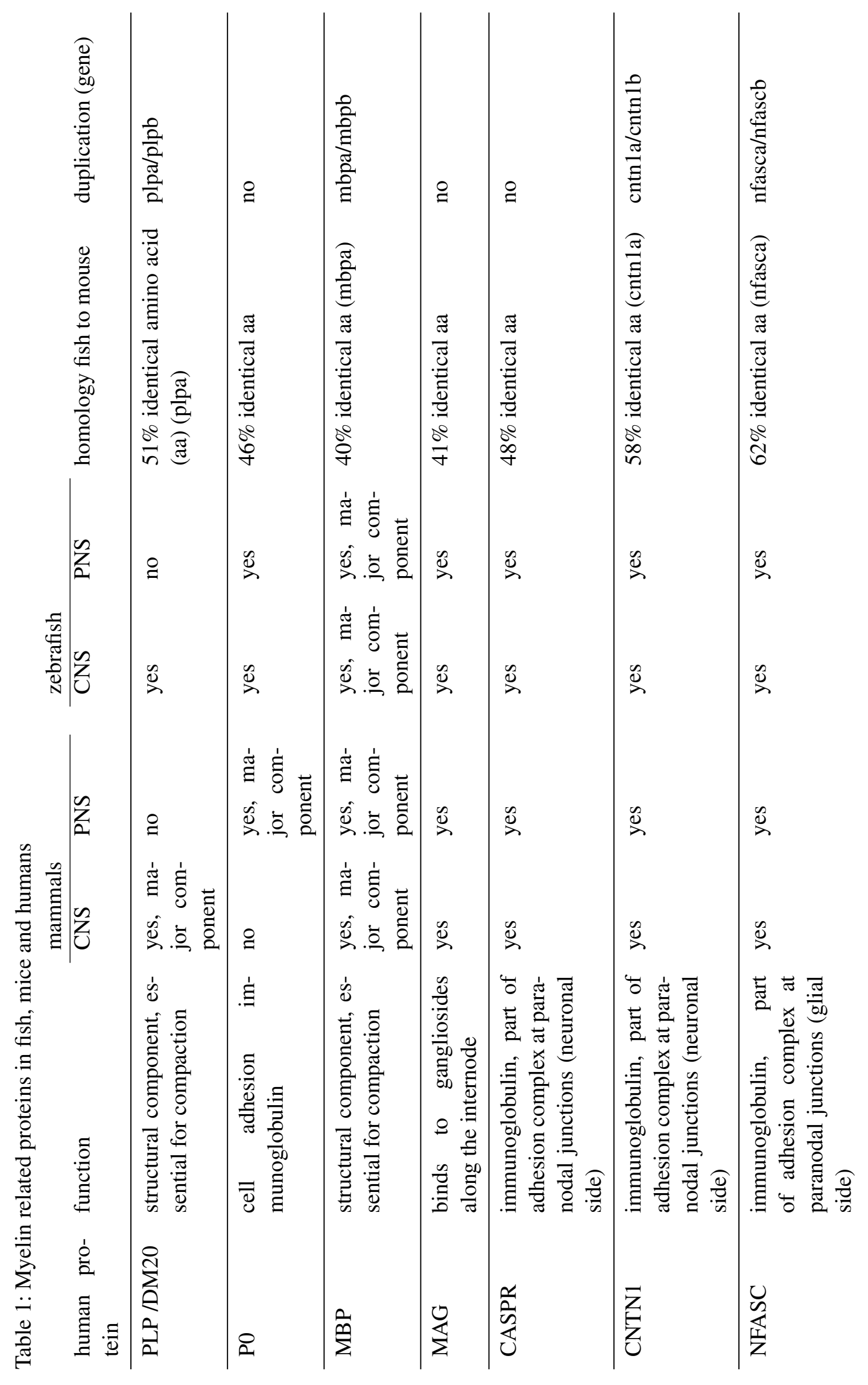




\subsection{Myelin in the peripheral and central nervous system}

Rennaissance physician Andries van Wesel (known as Vesalius) was dissecting brains from criminals and stolen corpses during the 16th century. He distinguished two macroscopic structures for the first time: grey and white matter [36]. The advances of neuroscience were and are still closely connected to the development of the instruments: from simple lenses to latest methods like confocal live-imaging, electron microscopy and super resolution techniques. The amazingly gifted hobby lense maker Antoni van Leeuwenhoek described the nerve as a tube in 1717, showing a great understanding of the anatomy. Later, light microscopes were able to discriminate between neurons and surrounding structures. Many known researchers as Ehrenberg, Schwann and Virchow contributed to the field. The latter of whom in 1854 , finally named the marrowlike substance in the core of the brain as myelin (Greek myelos $=$ marrow). Ten years later, Wilhelm and Werner von Siemens installed the first functional telegraph cable between Europe and America, allowing a historical breaktrough: transatlantic real-time communication. Obviously impressed by the technological innovation of his time, pathologist Louis-Antoine Ranvier compared myelin with the electrical insulators of the undersea cable [36].

However, Ranvier's analogy is beautiful but limited. Neuronal signal transmission differs entirely from pure flow of electrons in electric conductors. First of all, neurons consist of cell bodies, dendrites and the conducting axons. Even if some of them can reach the length of a meter, the signal is often given from neuron to neuron and every time shifted between electric transmission along axons and chemical transmission at synapses. Unlike in a copper cable, electrons are not free to flow in a current along the fiber. Furthermore, the signal is a moving membrane potential, transmitted by voltage-dependent ion channels, located at the axonal membrane. The charge between the axonal cell plasma and the sourrounding extracellular space (caused by gradients of ion concentration) plays a crucial role and not the charge between the poles of a power supply. Ranvier was the first one who suggested, myelin could be a fat cell pierced by, or wrapped around the axon. In the following years, the theory that axons secrete myelin was established. In the beginning 20th century, Rio-Hortega and his fellow Wilder Graves Penfield discovered 
oligodendroglia and hypothesized about their role in myelin formation. Ralph Stayner Lillie suggested the model of saltatory nerve conduction in the 1920s that was discovered experimentally in 1939 by Ichiji Tasaki, anesthetizing multiple nodes of Ranvier in a row and confirmed by Huxley and Stämpfli ten years later [37][38]. In 1953, Betty Ben Geren showed by electron microscopy, that Schwann cells are consisting of spirally wrapped lammella [39]. That CNSmyelin is a wound up extension of the oligodendrocyte cell membrane was than proven later on 1961 by Richard Paul and Mary Bartlett Bunge [40][41]. Later molecular analysis revealed that myelin contains only $40 \%$ of water and $70-85 \%$ of its dry mass belong to lipids (mainly cholesterol, sulfatides, plasmalogens and the myelin endemic cerebrosides). Compared to other tissues, the protein load is low [42].

With recent advances in molecular biology, genetic manipulations and microscopy, new insights of molecular interactions and mechanisms at the ultra-structure are in the scope for today's research.

\subsection{Generation of CNS myelin}

The brain of a newborn human is mostly unmyelinated - a new chance to form a brilliant mind. Myelination happens mainly during childhood but magnetic resonance imaging (MRI)-studies suggest, that it continues in humans until around 40 years of age. In contrast, gray matter volume is decreasing already from puberty on [43][44]. During aging CNS myelin is lost again, contributing to the decline of the whole CNS. Mechanisms of myelin turnover and remodeling are still elusive, but recent results showed that ablated oligodendrocytes can be replaced [45] and in mice life-long new formation of myelin sheaths can be observed [46].

In mice, myelination starts already in the embryonal phase and oligodendrocyte precursor cells (OPCs) are infiltrating the CNS in at least three waves [47]. The myelination process is complex: OPCs migrate to the later white matter regions, loose their ability to proliferate by differenting to myelinating oligodendrocytes followed by wrapping, thereby forming the internode. Myelination follows a strict hierarchical target pattern along increasing complexity: brain 
stem regions (responsible for basic processes) are myelinated first and heavily, whereas myelination ends at the later lightly myelinated association cortex (high intellectual functions) [48]. This is even reflected in human behavior: children start walking before they can talk or understand abstract concepts as mathematics. Furthermore, sensory tracts are myelinated before motor pathways, proximal before distal and projection before associative pathways [49]. The tight coordination hints towards a predetermined blueprint following genetic constrictions [50]. A smaller part of myelination, especially in behavior-related regions continues longer and can be influenced by external cues [51]. Mice without social contact show reduced adult myelination in the prefrontal cortex [52] and learning a language or motor-skill training (juggling and music instrument) induces white matter remodeling [53][54]. Therefore, myelin is accepted as an important factor influencing brain plasticity. Myelin is able to fine-tune neuronal circuits by subtle changes in the conduction velocity and to synchronize signals that travel over different distances [55]. On the other hand, aberrant myelination in the brain is contributing to psychiatric diseases as schizophrenia and depression [56][57].

When myelin is formed, oligodendrocytes and neurons engage in a very close and interdependent, livelong relationship. An oligodendrocyte is reaching out its processes, establishing contact to an axon that needs to be myelinated. It wraps layer by layer of membrane and generates a stable myelin sheath while recasting the axonal protein landscape and only leaving small gaps to the neighboring segments [58].

Oligodendrocytes are one of the most efficient producers of membrane in vertebrates. An average oligodendrocyte in the cortex or corpus callosum generates about $1-20 \times 10^{5} \mu \mathrm{m}^{2}$ of membrane [59] maintaining 30 to 80 myelin segments ranging from 20 to $200 \mu \mathrm{m}$ in length with a maximum of 60 layers [60][61]. Axon diameter, internode length and layer number depend on each other in a nearly linear relationship. The bigger the axon, the longer and thicker grows the internode [62][63]. Live-imaging in zebrafish revealed, that a myelin sheath can be formed within 5 hours but slowly grows together with the axon to compensate for body growth [64][45].

Myelination is a very complex process with many critical steps that, if gone wrong could put 
the fate of the whole structure on risk. Therefore, it is hard to believe that this process is not strictly regulated by multiple complementary (and mostly unknown) molecular mechanisms.

The generation of the myelin sheath can be structured in 3 steps: 1) Initiation, 2) Elongation and Compaction, 3) Termination:

\subsubsection{1) Initiation}

OPCs are migrating through the neuronal tissue and send out multiple processes scanning the environment for accessible axons. Forming sheaths is part of a differentiation program from OPCs to early myelinating and further to mature oligodendrocytes [47]. Once a process makes contact, it must confirm that its target is an axon that needs to be myelinated. General targeting mechanisms were identified: electrical activity, axon caliber and neurotransmitter release. Axons can attract oligodendrocytes with electrical activity (electrically active axons are more likely to be myelinated than anesthetized axons [65]) and restrict them by neurotransmitter release (endogenous gamma-aminobutyric acid (GABA) controls oligodendrocytes in cerebral cortical slices [66]). Even the sheath number per oligodendrocyte is regulated by electric activity and synaptic vesicle release [67]. Oligodendrocytes can approach axons thicker than $0.2 \mu \mathrm{m}$, but they myelinate large caliber axons first and on higher extent than smaller neighbors [17]. Axons of 0.2-0.8 $\mu \mathrm{m}$ caliber can be both, enwrapped or not. This inconsistency shows that axon size is a supportive, but not exclusive selection factor [68]. In vitro experiments showed, that oligodendrocytes can myelinate biochemically inactivated (paraformaldehyde (PFA) fixed) axons and even polystyrene fibers of correct size. However, if they have the choice, they prefer living axons [69][70]. In vivo, other cellular structures that fit the axonal diameter (like dendrites or cell bodies) need to be avoided. Axons of neocortical pyramidal neurons and interneurons are often partially myelinated with long unmyelinated distances [71] and the degree of myelination is even divergent in different brain regions, depending on the distance the signal needs to travel [72]. Altogether, this indicates the presence of signals that communicate to the oligodendrocytes where to myelinate and where to leave the gaps. 
Attractive, repulsive and permissive molecular cues were discussed and a few were identified: Inhibition of the neuron-specific cell adhesion molecules Nectin-like 1 (Necl1) results in a delay but not suppression of CNS myelination, suggesting just a minor role in initiation [73]. Nectinlike 4 (Nec14), a had no effect on myelination [74]. Junctional adhesion molecule B (Jam2) was identified to inhibit myelination of paired box protein 2 (PAX2)-positive dorsal horn neuronal cell bodies, indicating an inhibitory function of Jam2 to prevent mistargeting to neuronal cell bodies [75]. On the other hand, recent zebrafish data showed that myelin is simply mistargeted to cell bodies when axon numbers are reduced or myelin production is stimulated [76].

As most cells, oligodendrocytes and neurons are coated in negatively charged polysaccharides (glycocalyx) to conduct electrostatic repulsion. This repellent force needs to be overcome to get neuron and glia together [77].

It comes clear, that initiation of the myelin sheath works at a fragile equillibrium of membrane production, structure identification, guidance by surface molecules and retraction from falsely myelinated structures. Before a solid contact is established, oligodendrocytes have the opportunity to retract from the target, but molecular mechanisms of retraction are still elusive [78].

\subsubsection{2) Elongation and Compaction}

Once the initial contact is established, the myelin sheath grows in a spirally coordinated manner. But since the 'carpet crawler' model for peripheral nervous system (PNS) wrapping was introduced, many models for the CNS were proposed, all bound to their methodical limitations. Examples are: the 'liquid croissant', the 'serpent' or the 'yo-yo' model [79][80]. Detailed insights in myelin sheath formation and compaction were only possible with modern techniques of artifact-reduced fixation and three-dimensional electron microscopy [81]. Electron microscopy on high pressure frozen samples showed that membrane is fed in the system at the innermost layer (inner tongue). While the inner tongue is wrapping around the axon, the sheath expands laterally along the axon. At the same time compaction starts at the outer layers. During the whole 
process, every layer stays in close contact to the axon through the structures that become later the paranodal loops (see Fig.2). In an enrolled state, the myelin sheath would have a triangular shape with the long part close to the cell body and the shorter inner tongue close to the axon [82]. New membrane components are transferred to the innermost layer by cytoplasmic channels, that are well described in the PNS as Schmitt-Lantermann-incisures but for a long time were not seen in CNS internodes. Most CNS cytoplasmic channels are smaller than their PNS counterparts and close during maturation [83][82]. The paranodal adhesion complex (Caspr/Cntn1/Nfasc155) shows a spiralling localization around the axon during sheath development [80]. This indicates a role of this complex not just in formation and maintenance of the paranode but possibly also in the guidance of the lateral extension of the sheath. While growing, the single membrane layers engage in close contact with each other, get sealed together and push out the cytoplasma - a process that is called compaction. Myelin basic protein (Mbp) by changes of charge and conformation connects the oligodendroglial membranes from the cytosolic side (mayor dense line) and thereby seals the compacted myelin from the cytoplasma [84][85]. 2'-3'-cyclic nucleotide3'-phosphodiesterase (CNP) slows the process down and keeps cytoplasmic channels open, to guarantee traffic of nutrients and membrane [86]. PLP is a four-membrane spanning integral membrane protein contributing to the adhesion of the external layers of myelin and forming the interperiod line [87]. Another important function of PLP is its engagement in axonal support and sheath maintenance [88]. Moderate interaction between adjacent layers assure flexibility during the sheath generation [77].

\subsubsection{3) Termination}

It is crucial that sheath growth is terminated when two sheaths meet. Internodal length has a direct influence on conduction velocity by itself [89]. On the other hand, axon neighboring structures as the axon initial segment near the cell body and the axon terminal, as well as nodes of Ranvier, must stay open to guarantee proper saltatory nerve conduction. Myelin thickness is mainly dependent on the axonal diameter [62], whereas the sheath needs to adapt to the ex- 

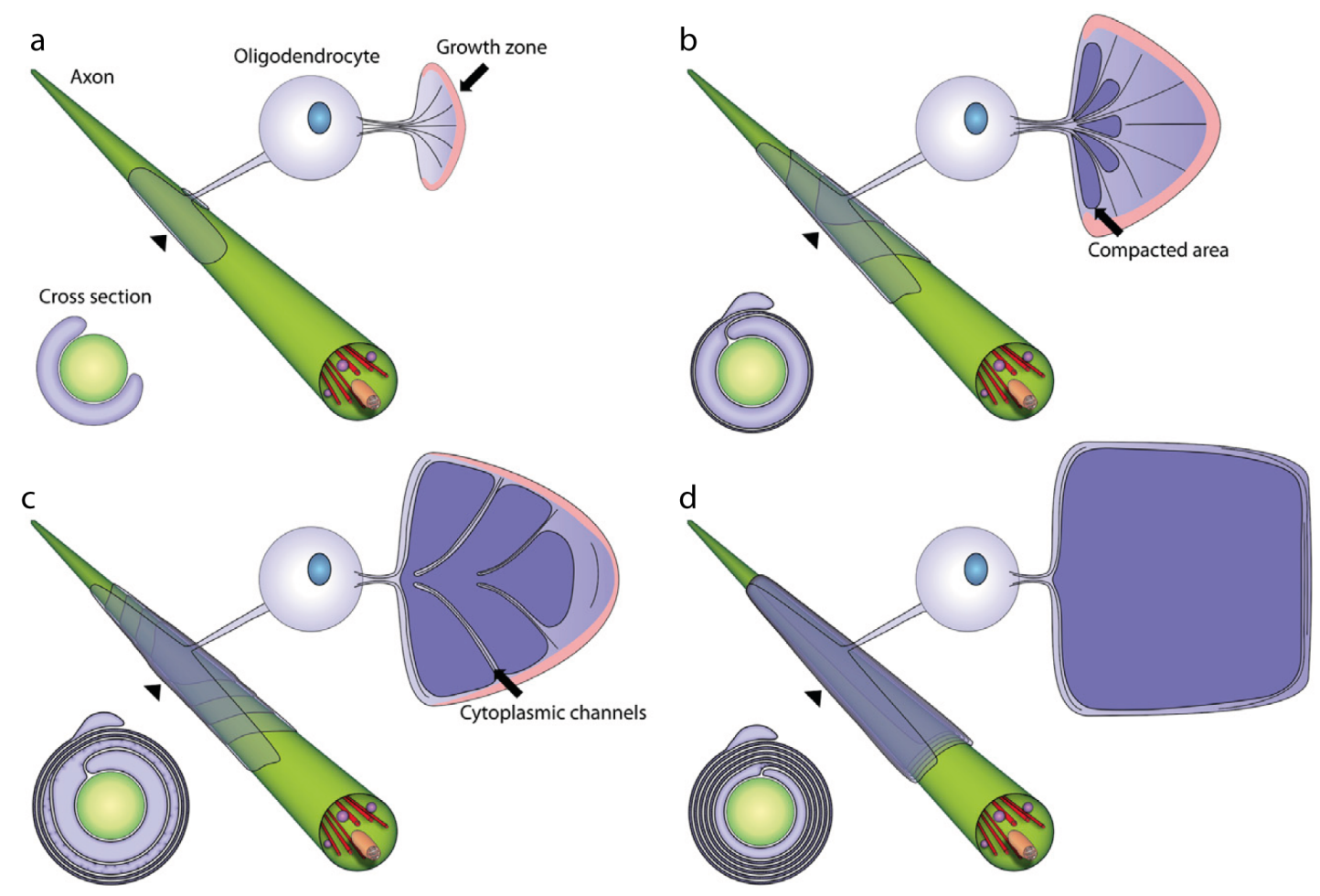

Figure 2: Model of CNS myelin sheath development (a-d) The model indicates the movement of the inner tongue and parallel compaction. The geometry of the membrane and the closing of cytoplasmic channels are demonstrated in the unwrapped representation. Figure from Snaidero et al. (Cell 2014), Elsevier, License number: 4393570735342 [82]

tension of myelinated axons during development of the organism. Cytoplasmic channels within the myelin sheaths are mainly needed for radial growth and get mostly reduced after sheath formation [86][82]. Laterally extending and adapting internodes were recently demonstrated in developing zebrafish [45]. The paranodal loops - a form of cytoplasmic channel by its own remain open, providing a connection to the biosynthetic machinery to support adaptive growth in sheath length.

Adhesion through cell surface molecules connected to the cytoskeleton could be one way of how to stop myelination when it is in the correct position. Mag in combination with the gangliosides GT1a and GD1b provides adhesion along the entire periaxonal surface [90]. Towards the node two adhesion complexes come into play: A) The juxtaparanodal complex of axonal Cntn2, 
axonal Caspr2 and glial Cntn2 forms a lateral diffusion barrier to cluster potassium channels. B) The paranodal complex of axonal Cntn1, axonal Caspr and glial Nfasc155 forms another diffusion barrier to restrict sodium channels to the nodes of Ranvier [91][92]. Both, Caspr and Caspr2 can interact with the axonal cytoskeleton via protein 4.1B providing a putative mechanism [93].

Formation of the node of Ranvier is considered as the final step in myelin sheath generation. Two glia-related mechanisms are working together to cluster the sodium channels: 1) nodal extra cellular matrix $(\mathrm{ECM}) / \mathrm{glia}$ derived Nfasc186 clustering; 2) constriction of the paranodal adhesion complex connected to $\beta$ IIspectrin. Both processes lead to a concentration of the scaffolds ankyrin $\mathrm{G}(\mathrm{AnkG})$ and $\beta \mathrm{IV}$ spectrin at the nodes of Ranvier and anchoring them to the axonal actin filaments. Since connected to it, opposing sheaths hinder their growth sterically via the axonal cytoskeleton [93].

Reports of animal models with distorted termination are rare. Hypermyelination in terms of thicker myelin (lower g-ratio) is reported for mutants influencing the AKT/mTOR-pathway, for example by enhancing AKT constitutively [94][95][96].

Knocking out phosphatase and tensin homolog (Pten) produces aberrant myelin structures as tomaculi in Schwann cells (focal hypermyelination) [97]. In rare cases, overgrown nodes and multiply myelinated axons have been seen in Mag or Caspr knock out mice. Neighboring sheaths are invading the node and some times even enter the next sheath, whereas the over-all myelin status in this single mutants stays intact [98][99][100]. Termination of sheath growth is an important part of the myelination program, but while initiation and growth phase are better understood, mechanisms of termination are mainly hypothetical.

\subsection{The structure of CNS myelin sheaths}

\subsubsection{The paranodal adhesion complex (CASPR/CNTN1/NFASC155)}

Each myelin membrane layer ends in a loop filled with cytoplasma that is in close contact with its axon at a structure called paranode. Since the myelinating inner tongue is spirally adding membrane from the inside, the oldest layer is the outermost, edging the node of Ranvier and the 


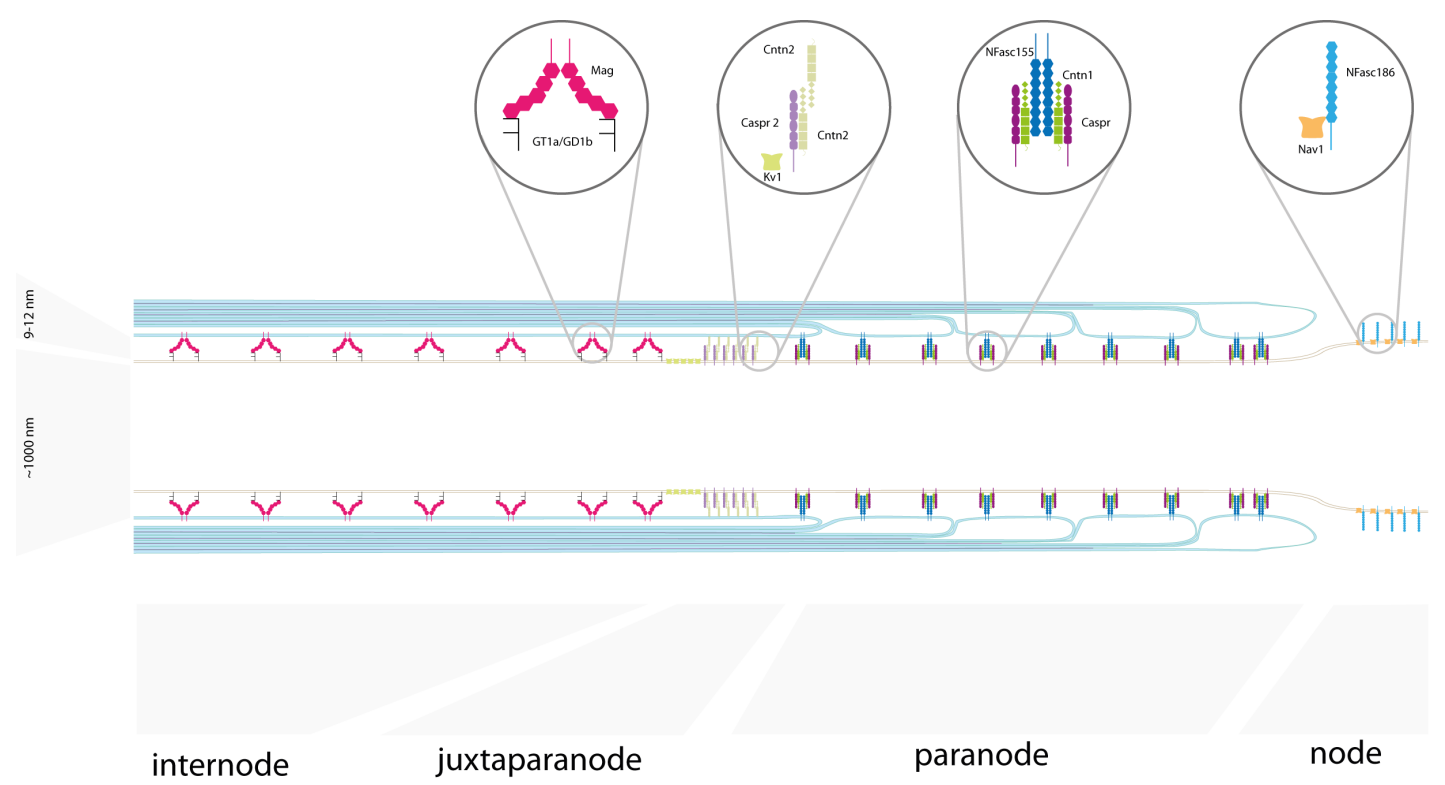

Figure 3: Molecular structure at the axon-oligodendroglial interface Mag is located at the internode, forms a dimer and binds to the gangliosides GT1a and GD1b on the neuronal side. Potassium channels (Kv1) are clustered at the juxtaparanodes by a complex of glial Cntn2 and neuronal Caspr2 and Cntn2. The paranodes are fixed by a complex of glial Nfasc155 and neuronal Caspr and Cntn1. Sodium channels (Nav1)are clustered at the nodes of Ranvier, together with Nfasc186.

last produced layer is the innermost forming the inner tongue. The number of paranodal loops depends on the number of wraps, but every layer stays in contact with the axon. In small and medium size myelin sheaths, the paranodal loops are the only non-compacted compartments providing a cytoplasmic connection to the oligodendrocyte [101]. This is a possible route for maintenance of the myelin sheath itself, for trophic support of the axon and lateral adaptive growth [16][101]. The tight paranodal junction is mainly formed by a protein complex consisting of Caspr and Cntn1 at the neuronal side and the Nfasc155 isoform at the oligodendroglial membrane. Since distributed along the internode, Mag is also localized to the paranodes [102].

Caspr (uniprot: O54991) is a $156 \mathrm{kDa}$, type I membrane spanning protein of the neurexin family. It presents 4 laminin G-like domains, 2 epidermal growth factor (EGF)-like domains, 1 fibrinogen-2 terminal and a F5/8 type C domain on its extracellular part. The intracellular C-terminus provides a SH3 binding domain. The Caspr protein is encoded by the Cntnapl gene 
located on chromosome 11 and has no isoform or splice variant. In the following, we will refer to the contactin associated protein gene as Cntnapl and to the protein as Caspr. Caspr can only leave the endoplasmatic reticulum (ER) and localize to the axonal membrane together with Cntn1 [103][104].

Cntn1 (uniprot: P12960) is a $113 \mathrm{kDa}$, glycosylphosphatidylinositol (GPI)-linked membrane protein of the immunoglobulin superfamily (contactin subfamily). It contains 6 immunoglobulinlike domains and 4 fibronectin type III domains. The Cntn1 protein is encoded by the Cntnl gene located on chromosome 15 and has no isoform or splice variant.

Nfasc (uniprot: Q810U3) is a type I membrane spanning protein of the immunoglobulin superfamily (L1/neurofascin/NgCAM subfamily). It contains 6 immunoglobulin-like domains and 4 fibronectin type III domains. Nfasc is encoded by the Nfasc gene located on chromosome 1 and has 3 splice variants named according to their molecular weight in rats. The neuronal variant Nfasc186 is clustering the sodium channels at the nodes of Ranvier, whereas the glial Nfasc155 is part of the paranodal complex. The third isoform is just a subtle modification of Nfasc155 [105]. By similarity, Nfasc is likely to form horseshoe-shaped homodimers in cis.

Functions of paranodal proteins were mainly studied in mouse mutants lacking them in various combinations. Mice lacking Caspr or Cntn1, suffer from similar symptoms: tremor, ataxia and motor impairments starting at P10 and limited lifespan (death around P23) [103]. Myelination in general is not affected, the number of myelinated axon, g-ratio and expression of myelin-related genes were unaltered, but the conduction velocity in the PNS is still reduced by 3 fold [100]. Defects become obvious at the cellular level: paranodal loops detach and potassium channels can enter the paranodal region [103]. In rare cases, myelin sheaths can even extend into the node and overlap their neighbors [100]. The symptom similarities between Cntnapl and Cntn1 null mice could be partially explained with the observation that Caspr needs Cntn1 to localize properly. Technically, Cntnl depletion leads to local knock down of Caspr (that without Cntn1 gets stuck in the cell body). Nfasc knock out mice die already at P6, but simultaneous expression of Nfasc186 delayed that. Disruption of the paranodal junction by selective knock out 
of Nfasc155 delays but does not stop myelination [105]. Caspr and Cntn1 do not localize to the paranodes when Nfasc is depleted. Localization is restored when a truncated form of Nfasc155 is expressed in oligodendrocytes. Expression of Nfasc186 could not rescue the localization of the adhesion complex to the paranodes [105]. This indicates that the oligodendrocyte induces the formation of the paranodal adhesion complex through Nfasc155, which in turn does not need to be connected to the glial cytoskeleton. Connection to the axonal cytoskeleton is essential for the paranodal junction. C-terminal truncated forms of Caspr are localized to paranodes during sheath growth, but are subsequently moving out of the paranodal region and redistributing along the axon, followed by Cntn1 [106]. Cntn1 binds to Caspr and thereby guiding its transport to the axonal membrane. During initiation and sheath growth, Caspr and Cntn1 bind to Nfasc155. Once this tripartite complex has reached the paranode, Caspr binds to protein 4.1B that can attach to $\alpha$ - and $\beta$ II-spectrin - the adaptors to the neuronal actin filament [107][106][108]. On the other side, glial AnkG can bind to Nfasc155 to facilitate node assembly [109][110].

The paranodal adhesion complex of Caspr, Cntn1 and Nfasc contributes to the formation and maintenance of the node of Ranvier and restricts the potassium channels at the juxtaparanodes. Caspr acts as a transmembrane scaffold to connect axon-glia adhesion (Cntn1 to Nfasc155) to the axonal cytoskeleton, building up a main structural requirement for the node of Ranvier.

\subsubsection{The juxtaparanodal adhesion complex (CASPR2/CNTN2/CNTN2)}

Juxtaparanodes are defined as the region between internode and paranode carrying clusters of potassium channels. The paranodal diffusion barrier separates the nodal sodium channels from the juxtaparanodal potassium channels, but correct clustering of the potassium channels is also dependent on juxtaparanodal adhesion molecules [111]. The juxtaparanodal adhesion complex consists of Contactin 2 associated protein (Caspr2) and Contactin2 (Cntn2) on the neuronal and Cntn2 on the glial side, together forming a second diffusion barrier around the axon.

Caspr2 (uniprot: Q9CPW0) is a $148 \mathrm{kDa}$, type I membrane spanning protein of the neurexin family. It presents 4 laminin G-like domains, 2 EGF-like domains, 1 fibrinogen-2 terminal and 
a F5/8 type $\mathrm{C}$ domain on its extracellular part. The intracellular $\mathrm{C}$-terminus is able to bind to KCNA2 (potassium channels). The Caspr2 protein is encoded by the Contactin 2 associated protein (Cntnap2) gene located on chromosome 6 and has no isoform or splice variant. In the following, we will refer to the contactin associated protein 2 gene as Cntnap2 and to the protein as Caspr2.

Cntn2 (uniprot: Q61330) is a $113 \mathrm{kDa}$, GPI-linked membrane protein of the immunoglobulin superfamily (contactin subfamily). It contains 6 immunoglobulin-like domains and 4 fibronectin type III domains. The Cntn2 protein is encoded by the Cntn2 gene located on chromosome 1 and has two isoforms by splice variant. Isoform 1 is involved in the juxtaparanodal adhesion complex, whereas isoform 2 resembles the intracellular $\mathrm{C}$-terminus. Isoform 2 is unable to bind to Cntn2, potentially fulfilling a regulatory role [112].

Mouse studies provided insights into putative functions of the Caspr2/Cntn2/Cntn2 juxtaparanodal adhesion complex. Cntnap $2^{-/-}$animals, in contrast to their Cntnap1 $1^{-/-}$counterparts, exhibited no severe impairments or neurological dysfunctions within the first 20 months. On the ultrastructural level, mutants were indistinguishable from wildtype controls in terms of: general myelin status, g-ratio and formation of nodes of Ranvier [111]. The only observation in Cntnap $^{-{ }^{--}}$mutants was the absence of potassium channel clustering at the juxtaparanodes, that did not influence the excitability of the nerve. Caspr2, similary as Caspr can bind to the cytoskeleton scaffold protein 4.1B (at least in the PNS) [113]. Cntnap2 depletion has no phenotypical consequences in mice, but in humans, Contactin 2 associated protein (CNTNAP2) mutations are associated to epilepsy and psychiatric diseases as autism and schizophrenia [114].

\subsubsection{Gangliosides and MAG interaction}

\section{Myelin Associated Glycoprotein (MAG) is an important myelin component}

MAG is the fifth most abundant myelin protein and exclusively expressed in myelinating oligodendrocytes (CNS) and Schwann cells (PNS) [115]. It consists of a cytoplasmic residue, a membrane spanning domain and five extracellular Immunoglobulin (Ig)-like domains. There- 
fore, MAG is classified as a single-pass type I membrane protein of the Ig superfamily. Due to the sialic-acid binding capacity, it belongs to the subgroup of sialic-acid-binding lectins (siglecs) [116]. The Mag gene provides two splice variants that differ only in the intracellular C-terminus. The larger L-Mag $(72 \mathrm{kDa})$ carries an intracellular tyrosin phosphorylation site that is missing in the smaller S-Mag $(67 \mathrm{kDa})$ isoform and could be part of a regulatory system [117]. The extracellular Ig-domains on the other hand, bind preferably to the gangliosides Gd1a and GT1b on the axonal side [118], with which it limits the gap between axon and the innermost myelin layer to 9-12 $\mathrm{mm}$ all along the internode [90]. Mag is already present at the early stages of the myelin sheath formation [119]. It subsequently localizes to the neuron-glia interface at the innermost layer of the myelin sheath, to the paranodal loops and to Schmidt-Lantermann-incisures in PNS-myelin [120].

\section{Mag depleted mice show no phenotype}

Two independent Mag-deficient mouse lines were published in 1989 and unexpectedly showed no severe phenotype [121][122]. Minor deficits in cognitive and locomotor skills were reported in 2-months old homozygous Mag animals, but obvious consequences are absent [116]. Only aging could provoke neurological dysfunctions, due to accelerated neurodegeneration. This leads to the assumption that Mag contributes to keep up the integrity of myelin over time [123]. Despite an up-regulation of Mbp in Schwann cells, the mayor myelin-related proteins (Proteolipidprotein (Plp), Neural cell adhesion molecule (N-cam), Neural cell adhesion molecule L1 (L1)) showed no altered expression levels in Schwann cells or oligodendrocytes and the composition of polar lipids remained the same [121][122][123].

Interestingly, depletion of Mag does not affect PNS-myelin in terms of myelination onset, axon caliber selection, myelin ultrastructure and nerve conduction [122]. In contrast, CNSmyelin shows mild hypomyelination, delay of myelination onset, smaller axon calibers and aberrant myelin structures along a generally normal appearing myelin [124]. The number and size of the oligodendrocyte cytoplasmic collar is reduced and patches of redundant myelin can be found. However, a visible but rare feature of the Mag mutant is the multiply myelinated axons. Surpris- 
ingly, the additional sheaths lacking axonal contact developed ordinary compacted myelin with normal periodicity and even paranodal-loop-like structures at the end [98]. Heterozygous Mag knock out animals can fully compensate and show no deformed myelin [98]. The combined double knock out of Mag and other myelin proteins (Fyn kinase (Fyn) [125], ceramide galactosyltransferase (CGT) [128] and Beta-1,4 N-acetylgalactosaminyltransferase 1 (Galgt1) [126] aggravated the myelin pathology. Due to these findings, many groups assumed that Mag plays an important role in neuron-glia recognition and sheath growth initiation [127].

\section{Mag can conduct cellular signals in two directions}

Two ways of signaling are postulated for Mag: acting as a ligand (myelin to axon) and acting as a receptor (axon to myelin). Being a ligand, the membrane spanning Mag and its soluble form dMag inhibit neurite outgrowth and induce axonal growth cone collapse by binding to sialic acids on the neuronal membrane [129][130]. Full length Mag can even interact independently of sialic acids [131]. Potential neuronal receptors are: Nogo-receptors 1\&2 [132][133], paired immunoglobulin-like receptor B [134] and beta-1-integrin [135]. This inhibitory influence on neurons is seen as one of the mayor problems hindering neurogenesis after neuronal injuries. On the other hand, when Mag is absent, myelinated axons of the PNS exhibit reduced calibers, suggesting a stimulating role of Mag for radial growth [136].

As a receptor on its own, the cytoplasmic part of Mag transducts signals most likely via its cytoplasmic domain and effectors like the Fyn kinase, that plays an important role in initiating CNS-myelination [137][138] and regulates the number of myelin sheaths per oligodendrocyte [64]. To understand the functions of the intracellular domain, it is worth to have a closer look on the Mag splice variants: L-Mag is the predominant isoform in the developing CNS myelin sheath. With sheath maturation, the S-Mag expression increases until an equilibrium of both isoforms is reached. Genetically modified mice that are expressing S-MAG and a C-terminaltruncated L-Mag (lacking the intracellular phosphorylation site), show the same features like the full length knock out animals: delayed compaction and multiply myelinated sheaths in CNSmyelin [139]. Together with the timing of expression, this suggests that L-Mag is the important 
isoform for sheath generation in the CNS, whereas the shorter S-MAG (lacking the receptor function) is sufficient for PNS sheath maintenance [139].

\section{Mutations in human MAG are connected to several disorders}

Mag is an essential component of human CNS-myelin and exhibits axon protective properties [140]. Malfunctioning of Mag due to genetic mutation or immune response has severe consequences for affected individuals. Dysregulation of Mag expression was reported in psychiatric disorders like autism [141] or schizophrenia [142]. Mag missense mutations are even worse: resulting in hereditary spastic paraplegia [143] or Pelizaeus-Merzbacher-like disease [144]. Antibodies against myelin proteins are a common feature of demyelinating diseases. They were found in samples of patients suffering from peripheral neuropathies [145] and multiple sclerosis (MS) [146].

\subsection{Myelin and cellular adhesion molecules in diseases}

Regarding its central role in the human nervous system, it is not surprising that myelin impairments have tremendous consequences for the affected organism. This is the case no matter if myelin is affected genetically or acutely.

The de novo generation of myelin can be affected by genetic mutations leading to severe problems for the patients from birth on (dysmyelination). Throughout the patients' lives, myelin generation, maintenance or function is impaired. Nerve conduction, axonal support and downstream mechanisms can be disturbed variously at different degrees [147]. The same functions can be compromised suddenly when healthy myelin is injured by physical, chemical or biological insults (demyelination). Acute brain damage leads often to oligodendrocyte death and subsequent neuronal loss. Auto-immune-based inflammation on the other hand, can destroy myelin slowly, causing severe neurological and psychological deficits progressing over time. Recently, the role of myelin in neurodegenerative diseases became a subject of research. Myelin's part in reacting, coping with, worsen or even causing certain neurodegenerative diseases is not understood for most of them [148][149]. 


\section{Leukodystrophies and genetic leukoencephalopathies: distortion of the paranodal junction}

About 1 in 7600 births is affected by a leukodystrophy. So far, the genetic causes often remained unclear, but improving diagnostic technology and high throughput sequencing led to the identification of responsible mutations [150]. Historically, neuropathies were diagnosed and classified according to the reported symptoms. Different mutations in the same gene can cause multiple diseases with varying spectra of symptoms.

Independent case reports of patients with mutations in one of the main components of the paranodal adhesion complex (Contactin 1 associated protein (CNTNAP1), Contactin1 (CNTN1) and Neurofascin (NFASC)) indicate comparable consequences like epilepsy, hypotonia, motor paralysis and early death. Individuals carrying homozygous mutations are severely affected, but even heterozygous mutations can cause tremendous impairments [151]. Missense, Nonsense, frameshift mutations and aberrant splice variants of the CNTNAP1 gene, for example, can cause Congenital Hypomyelinating Neuropathy (CHN). Many neonates carrying CNTNAP1 mutations die within their first year due to respiratory impairment. Individuals that grow older suffer from severe neurological deficits due to CNS hypomyelination and demyelination. Intellectual development is delayed and limited [152]. Another disease that is caused by CNTNAP1 mutations is Arthrogryposis Multiplex Congenita (AMC). Affected children show impairments of cranial nerves leading to orobulbar dysfunctions, facial nerve weakness, vocal cord paresis and joint contractures [153]. Most children suffering from CHN or AMC breath through a tracheostoma and get nurtured by a gastrostoma due to respiratory compromises and gastroesophageal reflux. Disruption of CNTN1, the binding partner of Contactin 1 associated protein (CASPR) leads to similar dysfunctions like CNTNAP1 mutations (called Lethal Congenital Myopathy (LCM)), but the consequences are even more severe and fatal. Homozygous children usually don't survive the first month after birth. Muscles are highly compromised, since the PNS and neuro muscular junctions (NMJs) are more affected than in the CNTNAP1 mutations [154]. Little is known about mutations in NFASC. Documented cases are rare, but the severe symptoms seem to be 
similar [155].

Interestingly, patients with mutations in the CNTNAP2 gene, coding for the juxtaparanodal protein Contactin 2 associated protein (CASPR2) show related but milder symptoms and additional psychological impairments like autism and intellectual retardation [114]. It is very likely, that due to further advances in sequencing diagnostics, increasing numbers of diseases related to proteins at the myelin-neuron interface will be identified.

\subsection{Aims of the study}

Myelination of axons is a crucial step during the development of the mammalian nervous system. Physical properties of the oligodendrocytes and the role of intracellular proteins like Myelin basic protein (MBP) during myelination were described previuosly [156][85]. The process of CNS myelination was reconstructed on the cellular level, demonstrating the dynamics of membrane organisation [82]. The aim of this study was to shed light on the role of intercellular adhesion molecules at the neuron-oligodendrocyte interface during myelin sheath growth. Based on our observations, we propose a model of synergistic interaction between the paranodal junction complex and Mag-dependend adhesion guiding and confining the myelinating leading edge (inner tongue) and thereby retain target selectivity. This study feeds the cellular model of CNS myelination with new molecular insights. 


\section{Materials and Methods}

\subsection{Materials}

\subsubsection{Buffer conditions}

\begin{tabular}{|c|c|c|}
\hline Reagent Name & USE & Ingredients \\
\hline E3-medium & fish larvae husbandry & $\begin{array}{l}5 \mathrm{mM} \mathrm{NaCl}, 0.17 \mathrm{mM} \mathrm{KCl}, 0.33 \mathrm{mM} \\
\mathrm{CaCl}_{2}, 0.33 \mathrm{mM} \mathrm{MgSO}_{4}\end{array}$ \\
\hline agarose gel & gel electrophoresis & $\begin{array}{l}1 \% \text { agarose in Tris-Acetat-EDTA buffer } \\
\text { (TAE) }\end{array}$ \\
\hline IF fixative & IF fixative for cells and tissue & $4 \%$ PFA in $1 x$ PBS \\
\hline $\begin{array}{l}\text { IF blocking so- } \\
\text { lution }\end{array}$ & $\begin{array}{l}\text { blocking and permeabiliza- } \\
\text { tion }\end{array}$ & $0.1 \%-0.5 \%$ triton-x-100, gelatine, $1 \mathrm{x}$ PBS \\
\hline $\begin{array}{l}\text { IF washing so- } \\
\text { lution }\end{array}$ & washing & $0.2 \%$ tween-20 in $1 \times$ PBS \\
\hline $\begin{array}{l}\text { PBS with } \\
\text { gelatine and } \\
\text { triton-x- } \\
100(\text { PBS-GT) }\end{array}$ & $\begin{array}{l}\text { long protocol blocking and } \\
\text { permeabilization }\end{array}$ & $1 \%$ triton- $\mathrm{x}-100,0.2 \%$ gelatine in $1 \mathrm{x}$ PBS \\
\hline $\begin{array}{l}\text { Karlsson- } \\
\text { Schultz }\end{array}$ & EM fixative & $\begin{array}{l}2.5 \% \text { glutaraldehyde, } 4 \% \text { PFA in phos- } \\
\text { phate buffer } \mathrm{pH}=7.3\end{array}$ \\
\hline EPON & EM embedding & $\begin{array}{l}21.4 \mathrm{~g} \text { Glycidether } 100,14.4 \mathrm{~g} \text { DDSA, } \\
11.3 \mathrm{~g} \text { MNA, } 0.84 \mathrm{ml} \text { DMP-30 }\end{array}$ \\
\hline $\begin{array}{l}\text { contrast solu- } \\
\text { tion }\end{array}$ & EM contrasting & $\begin{array}{l}4 \% \text { uranyl acetate in water, filtered with } \\
0.22 \mu \mathrm{m} \text { filter }\end{array}$ \\
\hline EM fixative fish & fixative & $\begin{array}{l}2.5 \% \text { glutaraldehyde, } 4 \% \text { PFA in cacody- } \\
\text { olate buffer } \mathrm{pH}=7.4\end{array}$ \\
\hline
\end{tabular}




\begin{tabular}{|c|c|c|}
\hline OHSC-medium & OHSC culture medium & $\begin{array}{l}50 \% \mathrm{MEM}, 5 \% \mathrm{BME} \text { (Earle's salts), } 25 \% \\
\text { horse serum, } 1 \% \text { GlutaMAX supplement, } \\
0.648 \% \text { glucose, pH-adjustment to } 7.2 \text { us- } \\
\text { ing } 7.5 \% \mathrm{NaHCO}_{3} \text {, sterile filtered }(0.22 \\
\mu \mathrm{l})\end{array}$ \\
\hline medium A & OHSC preparation medium & $\begin{array}{l}15 \mathrm{mM} \text { HEPES, } 0.57 \% \text { glucose in } 500 \mathrm{ml} \\
\text { HBSS }\end{array}$ \\
\hline BME-Medium & $\begin{array}{l}\text { Oligodendrocyte extraction } \\
\text { medium }\end{array}$ & $\begin{array}{l}500 \mathrm{ml} \text { BME-Medium, } 10 \% \text { horse serum, } \\
1 \% \text { Penicillin/Streptomycin, } 1 \% \text { Gluta- } \\
\max \end{array}$ \\
\hline Super-SATO & $\begin{array}{l}\text { Oligodendrocyte culture } \\
\text { medium }\end{array}$ & $\begin{array}{l}100 \mathrm{ml}=80 \mathrm{ml} \text { DMEM (high glucose), } \\
2 \mathrm{ml} \text { B27-Supplement, } 1 \mathrm{ml} \text { Glutamax, } \\
0.5 \mathrm{ml} \text { Penicilin/Streptomycin, } 1 \mathrm{ml} \text { Horse } \\
\text { Serum, } 1 \mathrm{ml} \text { pyruvate soulution, } 10 \mu 1 \text { Tri- } \\
\text { iodothyronine solution, } 13 \mu \mathrm{l} \text { L-Thyroxine } \\
\text { solution }\end{array}$ \\
\hline
\end{tabular}

\subsubsection{Antibodies}

\begin{tabular}{lllll}
\hline Antibody & Target & Species & Dilution & Company \\
\hline Caspr & Contactin associated protein & ms IgG1 & $1: 1000$ & Neuromab \\
Cntn1 & Contactin 1 & ms IgG1 & $1: 100$ & Neuromab \\
Nfil & Neurofilament & chicken IgG & $1: 2000$ & abcam \\
Mbp & Myelin basic protein & rabbitt IgG & $1: 1000$ & Dako \\
AnkG & Ankyrin G & mouse IgG2a & $1: 250$ & Neuromab \\
Nav1.6 & Sodium Channel 1.6 & rabbitt IgG & $1: 200$ & Alomone \\
\hline
\end{tabular}




\subsubsection{Vectors}

\begin{tabular}{|c|c|c|c|c|}
\hline Vector & Species & Resistance & Use & Provider \\
\hline pME-polyA & $\mathrm{dr}$ & kan & cloning & Tol2Kit [22] \\
\hline p5E-Olig1(3.2kb) & $\mathrm{dr}$ & kan & cloning & $\begin{array}{l}\text { was a gift from Tim } \\
\text { Czopka }\end{array}$ \\
\hline p3E-polyA & $\mathrm{dr}$ & kan & cloning & Tol2Kit [22] \\
\hline pDONR221 & $\mathrm{dr}$ & kan & cloning & Invitrogen \\
\hline pDONR_Nfascb & $\mathrm{dr}$ & kan & cloning & self generated \\
\hline pDestTol2pA2 & $\mathrm{dr}$ & amp & cloning & Tol2Kit [22] \\
\hline pDestTol2CG2 & $\mathrm{dr}$ & amp & cloning & Tol2Kit [22] \\
\hline pDestTol2pA2-U6:gRNA & $\mathrm{dr}$ & amp & cloning & $\begin{array}{l}\text { was a gift from Leonard } \\
\text { Zon (Addgene plasmid } \\
\text { \#63157) [23] }\end{array}$ \\
\hline $\begin{array}{l}\text { pTol2_cg2_UAS_ } \\
\text { Nfascb_GFP }\end{array}$ & $\mathrm{dr}$ & amp & Nfascb localization & self generated \\
\hline $\begin{array}{l}\text { pTol2_cg2_UAS_ } \\
\text { Nfascb_mcherry }\end{array}$ & $\mathrm{dr}$ & amp & Nfascb localization & self generated \\
\hline $\begin{array}{l}\text { pTol2_cg2_UAS_ } \\
\text { Caspr_YFP }\end{array}$ & $\mathrm{dr}$ & amp & Caspr localization & $\begin{array}{l}\text { generated by Martina } \\
\text { Arends }\end{array}$ \\
\hline $\begin{array}{l}\text { pTol2_gRNA_Olig1(3.2)_ } \\
\text { Cas9_T2A_RFPcaax }\end{array}$ & $\mathrm{dr}$ & amp & cloning & self generated \\
\hline $\begin{array}{l}\text { pTol2_Magko_Olig1(3.2)_ } \\
\text { Cas9_T2A_RFPcaax }\end{array}$ & $\mathrm{dr}$ & amp & $\begin{array}{l}\text { conditional Mag } \\
\text { knock out }\end{array}$ & self generated \\
\hline pTol2_huc_KalTA4 & $\mathrm{dr}$ & amp & $\begin{array}{l}\text { promoter for spe- } \\
\text { cific expression }\end{array}$ & $\begin{array}{l}\text { was a gift from Tim } \\
\text { Czopka }\end{array}$ \\
\hline
\end{tabular}




$\begin{array}{lllllll}\text { pTol2_Olig1_KalTA4 } & \text { dr } & \text { amp } & \text { promoter for spe- was a gift from Tim } \\ & & & \text { cific expression } & \text { Czopka } \\ \text { pTol2_Sox10_KalTA4 } & \mathrm{dr} & \text { amp } & \text { promoter for spe- was a gift from Tim } \\ & & & \text { cific expression } & \text { Czopka } \\ & & & \end{array}$

\subsubsection{Chemicals}

\begin{tabular}{|c|c|}
\hline Reagent & Company \\
\hline Acrylamide & Sigma-Aldrich, Steinheim \\
\hline Agarose UltraPure Low Melting Point & Invitrogen, \\
\hline Agarose peqGOLD Universal-Agarose & PeqLab, \\
\hline Ampicillin & Roth, Karlsruhe \\
\hline Calcium chlorid-Dihydrat & Merck Chemicals, Darmstadt \\
\hline Cas9 protein (recombinant) & Sigma-Aldrich, Steinheim \\
\hline Di-sodiumhydrogenphosphate & Roth, Karlsruhe \\
\hline EDTA (Ethylenediaminetetraacetic acid) & Sigma-Aldrich, Steinheim \\
\hline Ethanol $99.9 \%$ & Roth, Karlsruhe \\
\hline Ethylene Glycol & Sigma-Aldrich, Steinheim \\
\hline Glucose & Sigma-Aldrich, Steinheim \\
\hline Glycine & Sigma-Aldrich, Steinheim \\
\hline Hydrochloric acid & Sigma-Aldrich, Steinheim \\
\hline \multirow[t]{2}{*}{ Immersol W2010 } & Carl Zeiss Forschung und Technologie \\
\hline & Chemie, Oberkochen \\
\hline Isopropanol & Roth, Karlsruhe \\
\hline Kanamycinsulfat & Roth, Karlsruhe \\
\hline Ketamine $10 \%$ & Bremer Pharma GmbH, Warburg \\
\hline Methanol $100 \%$ & Roth, Karlsruhe \\
\hline
\end{tabular}


Mowiol

Paraformaldehyde

Prolong Diamond Antifade Mountant

Pronase

Proteinase K PCR grade

1-phenyl-2-thiourea

Rompun $2 \%$

Sodium chloride

Tissue Freezing Medium, TissueTec OCT

Tricaine (3-amino benzoic acidethylester)

TRIS (Tris(hydroxymethyl)-aminomethan)

Triton X-100

tween-20

type F immersion oil
Roth, Karlsruhe

Sigma-Aldrich, Steinheim

Invitrogen/Thermo Fisher, Eugene, OR (USA)

Sigma-Aldrich, Steinheim

Sigma-Aldrich, Steinheim

Sigma-Aldrich, Steinheim

Bayer, Leverkusen

Roth, Karlsruhe

Sakura Finetek, Torrance, CA (USA)

Sigma-Aldrich, Steinheim

Roth, Karlsruhe

Sigma-Aldrich, Steinheim

Merck Chemicals, Darmstadt

Leica Microsystems, Wetzlar

\subsubsection{Kits}

\begin{tabular}{lll}
\hline Use & Product & Company \\
\hline DNA extraction from tissue & Invisorb Spin DNA extraction kit & Stratec, Birkenfeld \\
DNA extraction from agarose gel & Monarch DNA gel extraction kit & NEB, Ipswich, MA (USA) \\
Plasmid preparation from E.coli 5ml & Plasmid quick pure & Macherey \& Nagel, Düren \\
Plasmid preparation from E.coli 100ml & Nucleobond Xtra EF Midi & Macherey \& Nagel, Düren \\
\hline
\end{tabular}




\subsubsection{Instruments}

\begin{tabular}{|c|c|c|}
\hline Machine & Model & Company \\
\hline Centrifuge & Biofuge pico & Heraeus Instruments, Hanau \\
\hline Centrifuge & Centrifuge 5415R & Eppendorf, Hamburg \\
\hline Cryostat & & $\begin{array}{l}\text { Thermo Fisher Scientific Inc., } \\
\text { Waltham, MA (USA) }\end{array}$ \\
\hline $\begin{array}{l}\text { Confocal laser scanning } \\
\text { microscope }\end{array}$ & Leica TCS SP5 & Leica, Wetzlar \\
\hline $\begin{array}{l}\text { Confocal laser scanning } \\
\text { microscope }\end{array}$ & Leica TCS SP8 & Leica, Wetzlar \\
\hline Diamond Knife & Ultra $45^{\circ}$ & DiATOME, Nidau, Switzerland \\
\hline Electrophoresis device & $\begin{array}{l}\text { MPI ExMed technical ser- } \\
\text { vice }\end{array}$ & MPI ExMed, Göttingen \\
\hline Freeze substitution device & $\begin{array}{l}\text { Leica AFS II, Leica, Wet- } \\
\text { zlar }\end{array}$ & \\
\hline High pressure freezer & Leica HPM100 & Leica, Wetzlar \\
\hline Incubator (bacteria) & certomat $\mathrm{H}$ & B. Braun Melsungen AG, Melsungen \\
\hline $\begin{array}{l}\text { automated tissue proces- } \\
\text { sor }\end{array}$ & Lynx II & $\begin{array}{l}\text { Electron Microscopy Science, Hatfield, } \\
\text { PA (USA) }\end{array}$ \\
\hline $\begin{array}{l}\text { Magnetic stirrer and } \\
\text { heater }\end{array}$ & RH Basic 2 & IKA, Staufen \\
\hline Microwave & Pelco BioWave Pro+ & Ted Pella, Redding, CA (USA \\
\hline Moving platform & 3005 & GFL, Burgwedel \\
\hline pH-meter & Seven Compact & Mettler Toledo, Gieen \\
\hline Perfusion pump & PERIPRO-4LS & $\begin{array}{l}\text { World Precision Instruments, Sarasota, } \\
\text { FL (USA) }\end{array}$ \\
\hline
\end{tabular}




\begin{tabular}{|c|c|c|c|}
\hline \multicolumn{2}{|l|}{ Pipette boy } & Easypet & Eppendorf, Hamburg \\
\hline \multirow{2}{*}{\multicolumn{2}{|c|}{ Pipettes }} & Research Plus $(2.5 \mu 1,10$ & Eppendorf, Hamburg \\
\hline & & $\mu \mathrm{l}, 100 \mu \mathrm{l}, 1000 \mu \mathrm{l})$ & \\
\hline \multicolumn{2}{|l|}{ Power source } & BioRad PowerPac Basic & BioRad, München \\
\hline \multicolumn{2}{|c|}{ Spectrophotometer } & NanoDrop ND-1000 & Peqlab Biotechnologie, Erlangen \\
\hline \multicolumn{2}{|l|}{ Thermomixer } & Thermomixer compact & Eppendorf, Hamburg \\
\hline \multicolumn{2}{|l|}{ Tissue chopper } & Mcllwain TC752 & Campden Instruments, UK \\
\hline \multirow{2}{*}{\multicolumn{2}{|c|}{$\begin{array}{l}\text { Transmission } \\
\text { microscope }\end{array}$}} & LEO 912 Omega electron & Zeiss, Oberkochen \\
\hline & & microscope & \\
\hline \multirow{2}{*}{\multicolumn{4}{|c|}{$\begin{array}{l}\text { Transmission } \\
\text { microscope }\end{array}$}} \\
\hline & & & \\
\hline \multicolumn{2}{|l|}{ Ultramicrotome } & Leica Ultracut $\mathrm{S}$ & Leica, Wetzlar \\
\hline \multicolumn{2}{|l|}{ Vortex } & vortex-Genie 2 & $\begin{array}{l}\text { Scientific Industries, New York, NY } \\
\text { (USA) }\end{array}$ \\
\hline \multicolumn{2}{|l|}{ Water supply } & Arium lab water system & Sartorius, Gttingen \\
\hline
\end{tabular}

\subsubsection{Consumables}

\begin{tabular}{ll}
\hline Product & Company \\
\hline 6-well-Platte & Nunc GmbH \& Co. KG, Langenselbold \\
24-well-Platte & Nunc GmbH \& Co. KG, Langenselbold \\
48-well-Platte & Nunc GmbH \& Co. KG, Langenselbold \\
Cannula & B. Braun Melsungen AG, Melsungen \\
Cell scraper & Sarstedt, Nümbrecht \\
Glass pipettes & \\
Imaging dishes glass bottom 35mm \#1.5 & cellvis, Mountain View, CA (USA) \\
Microscope slides superfrost Menzel & Thermo Fisher, Eugene, OR (USA)
\end{tabular}


Reaction tubes

Serological pipette $(5 \mathrm{ml}, 10 \mathrm{ml})$

Syringes

B. Braun Melsungen AG, Melsungen

\subsubsection{Software}

\begin{tabular}{lll}
\hline Product & Use & Company \\
\hline Adobe Illustrator CS5 & generation and processing of figures & Adobe, San Jose, CA (USA) \\
FIJI & image processing & open source [159][160][161] \\
Imaris v9.2 & sheat length measurement in 3D & bitplane, Zürich (Switzerland) \\
LAS X & image aquisition and processing & Leica, Wetzlar \\
LaTeX/TeXnicCenter & text processing and layout & open source \\
microscopy imaging browser (MIB) & 3D EM reconstruction & open source [162] \\
$\mathrm{R}$ & statistical analysis & open source [163] \\
\hline
\end{tabular}




\subsection{Methods}

\subsubsection{Mouse husbandry}

Mice were housed at the MPI of experimental medicine Göttingen and the DZNE in München. The cages were part of a ventilated rack system and were embedded with woodchips and enriched with a piece of wood and a house. The rooms were kept at $20^{\circ} \mathrm{C}$ to $24^{\circ} \mathrm{C}$ and $45 \%$ to $65 \%$ relative humidity. Light cycle: $12 \mathrm{~h}$ dark/12h light. Mice had access to water and food ad libitum. Mice for experimental purposes (Caspr/Caspr2/Mag triple knock out line) were tattooed at P5 and genotyped by tail biopsies. Mice for breeding were genotyped using ear clips from P21.

Caspr/Caspr2 knock out mice (NMRI background) were kindly provided by Elior Peles (Weizmann Institute, Rehovot, Israel). Mag knock out mice (C57/B6J background) were kindly provided by Hauke Werner (MPI ExMed, Göttingen). Cntn1 knock out mice were purchased from Jackson Laboratories. All experiments on animals were executed according to the regulations of the states of Niedersachsen and Bayern. (License number: Niedersachsen - AZ: 14/1729; Bayern - AZ: 55.2-1-54-2532-161-2016)

\subsubsection{Mouse genotyping}

Mouse deoxynucleic acid (DNA) was extracted using the Stratec Invisorb Spin DNA extraction kit according to the manufacturer's guidelines (elution voulme $=50 \mu \mathrm{l}$ ). $1 \mu \mathrm{l}$ of extracted DNA was used for polymerase chain reaction (PCR) in a Biometra PCR-cycler according to the protocols in Tab.12 and Tab.10 using the primers in Tab.15. $10 \mu$ of DNA were mixed with loading dye (6x) and applied on a 1\% agarose gel and gel electrophoresis was conducted at 140V for 1 h. Gel was scanned with a BioRad GelDoc XR+. 


\subsubsection{Immunohistochemistry of mouse tissue - conventional cryosectioning protocol}

Mice were anesthetized by injecting $0.2 \mathrm{mg}$ xylazin and $3 \mathrm{mg}$ ketamin in $0.9 \%$ aqueous $\mathrm{NaCl}-$ solution per g body weight and perfused with phosphate buffered saline (PBS) for 15 min following 2\% or 4\% PFA in PBS for 15 min. Spinal cord, optic nerve and brain were removed, fixed in $2 \%$ or $4 \%$ PFA in PBS over night and transferred to $30 \%$ sucrose solution. After two days, the tissue was embedded in optimal cutting temperature compound (OCT) and stored at $-80^{\circ} \mathrm{C}$. Longitudinally optic nerve and spinal cord sections $(20 \mu \mathrm{m})$ were cut to $30 \mu \mathrm{m}$ slices, using a Leica cryostat. Sections were stored free-floating in cryoprotective solution. For stainings, sections were carefully transferred to a 24 well plate, using a brush. After washing twice with PBS, $200 \mu \mathrm{l}$ blocking solution containing $0.5 \%$ triton-x-100 for permeabilization, was applied for 1 hour. All antibodies were diluted in blocking solution containing triton-x-100, according to their working concentration. Sections were treated with the antibody solution over night at $4{ }^{\circ} \mathrm{C}$, smoothly shaken. Next day, sections were washed 3 times with PBS before secondary antibodies (1:1000) were added and incubated for 1 hour at room temperature. Sections were washed again, mounted to glass slides using Prolong Diamond antifade mountant and dried over night. Images were acquired using Leica SP5 and Leica SP8 confocal laser scanning microscopes.

\subsubsection{Immunohistochemistry of mouse tissue - vibratome, long incubation protocol}

Brains were fixed in PFA as described before. Fixed brains were cut in sections of $150 \mu \mathrm{m}$ with a Leica vibratome. Sections were stored in a multi-well plate in PBS at $4{ }^{\circ} \mathrm{C}$. For stainings, sections were carefully transferred to a $1.5 \mathrm{ml}$ tube, using a brush. Sections were incubated in PBS-GT at $37^{\circ} \mathrm{C}$ over night for blocking. Antibodies were diluted in PBS-GT and added to the slices. Samples were incubated for 3 days at $37^{\circ} \mathrm{C} .6$ PBS-GT washing steps (30 min) were applied and sections were incubated with secondary antibodies for another 2 days. Sections were washed again 6 times, mounted to glass slides using Prolong Diamond antifade mountant and 
dried over night. Images were acquired using a Leica SP8 confocal laser scanning microscope, with a $63 x$ objective $(1.4$, immersion oil type $\mathrm{F})$ using photomultiplier detectors, line averaging of $4,1024 \times 1024$ pixels and the pinhole at 1 airy unit. Myelin sheaths of the thalamus were measured in 3D using IMARIS (bitplane).

\subsubsection{Expansion microscopy}

Expansion microscopy was conducted according to Chen et al. [166]. PFA-fixed longitudinal spinal cord sections were stained with primary antibody as described before (chapter 2.2.3). Secondary antibodies conjugated with a single stranded DNA oligonucleotide were used for secondary labeling. Subsequently, samples were incubated with complementary oligonucleotides carrying a fluorophore and a methacryloyl group. The sample was infused in acrylamide, transferred to a gelling chamber and allowed to polymerize. (The methacryloyl integrates to the acrylamid mesh, immobilizing the fluorophore at the position of the protein.) Proteinase K was added and incubated to digest all proteins in the sample. Subsequently, desalted water was added to expand the gel. Soon after, the sample was imaged using a Leica SP5 confocal microscope. Expansion rates can only be estimated, therefore no scale bar can be provided. Estimated from the size of the node of Ranvier, we reached an expansion factor of about $100 \%$.

\subsubsection{Zebrafish husbandry}

Zebrafish were housed at the DZNE fish facility in München. Adult fish were mated in a separated breeding box over night. Fertilized eggs were separated, washed and incubated in petri dishes filled with $\mathrm{E} 3$ medium at $28^{\circ} \mathrm{C}$. At day 1 after fertilization, healthy embryos were sorted and disinfected using $0.04 \%$ bleach in water two times for $5 \mathrm{~min} .10 \mu \mathrm{l}$ of pronase were added per dish to support hatching. After another two days of incubation, 30 fish each were transferred to 3.51 tanks. Zebrafish were kept at $28^{\circ} \mathrm{C}$, constant waterflow and a light cycle of $12 \mathrm{~h} \mathrm{light}$ and $12 \mathrm{~h}$ dark with simulated sunrise and sunset. The water has a salt concentration of 500-1000 microSi and $\mathrm{pH}$ of 7.5. At the age of 3 months the fish were genotyped by taking fin clip biop- 
sies. According to their genotypes, the fish were split up to 3.51 tanks, to a maximum of 17 fish per tank. Adult fish were fed twice with powdered food by an automated system and once with artemia. Fish larvae for experiments were treated with $600 \mu \mathrm{l}$ PTU per dish at $1 \mathrm{dpf}$, to prevent pigmentation. (License number: Bayern - AZ: 55.2-154-2532-157)

\subsubsection{Zebrafish genotyping}

Zebrafish larvae or fin clips were lysed in Tris-EDTA buffer (TE) $(\mathrm{pH}=8.0)$ and proteinase $\mathrm{K}(1 \%$ of $17 \mathrm{mg} / \mathrm{ml}$ ) for $8 \mathrm{~h}$ at $55^{\circ} \mathrm{C}$. Proteinase $\mathrm{K}$ was inactivated at $65^{\circ} \mathrm{C}$ for $10 \mathrm{~min} .1 \mu \mathrm{l}$ of lysate was used for PCR according to the protocols in Tab.13 and Tab.11 using the primers in Tab.16. PCR product was digested with individual restriction enzymes for $1 \mathrm{~h}$ at $37^{\circ} \mathrm{C}$ and applied on a $1 \%$ agarose gel for gel electrophoresis at $140 \mathrm{~V}$ for $1 \mathrm{~h}$ (see Tab.14). Gel was scanned with a BioRad GelDoc XR+ gel imager.

\subsubsection{Generation of mutant zebrafish}

Clustered regularly interspaced short palindromic repeats/CRISPR-associated 9 (Crispr/Cas9) gene editing was recently adapted to modify model organisms including zebrafish [23]. We identified several candidate guide ribonucleic acid (gRNA) sequences (20-23 nucleotides (nt)) per gene localized at different exons and around palindromic restriction sites of known restriction enzymes using the chopchop web tool [164][165]. PCR primer at about $200 \mathrm{nt}$ up and downstream of the target were designed and ordered from Thermo Fischer Scientific. PCR of fish complementary deoxynucleic acid (cDNA) was performed according to standard 3-step protocols. Primer combinations with satisfying PCR products were selected. 2-3 gRNA sequences were selected by PCR product quality and availability of the restriction enzymes and ordered from Integrated DNA Technologies. 25 pg candidate gRNAs were injected in fertilized embryos of the one-cell stage (Tg(mbp:eGFP_CAAX)), together with recombinant Cas9 protein. Fish were bleached at 3 days post fertilization (dpf) and released to 3.51 tanks at $5 \mathrm{dpf}$. The founder generation (F0) was raised and kept at the automated fish feeding system at the DZNE München. 
At the age of 3 months, founders were mated with wildtype $\operatorname{Tg}(\mathrm{mbp}$ :eGFP_CAAX) individuals. F1 egglays were partially genotyped at $3 \mathrm{dpf}$ to check for germline transmission. Egglays containing mutations were bleached and raised. F1 fish were genotyped at the age of 3 months by fin clipping, PCR and subsequent enzyme digestion. The respective exon was sequenced and animals carrying the same frame-shift mutation were bred to obtain the F2 generation containing wildtype (1/4), heterozygous (1/2) and homozygous mutant (1/4) animals. Genotypes were distributed as expected from mendelian inheritance. If possible, homozygous animals were used for breeding (only mag(-/-)). For the generation of double mutant mice, F1 animals of the respective lines were cross bred obtaining double heterozygous mutants in 1 out of 16 animals. Due to physical impairment, double mutants were kept as cntn1(+/-)mag(-/-) and cntnap1(+/-)mag(-/-) to generate animals for experiments. cDNA of homozygous single mutant fish was analyzed by quantitative real-time polymerase chain reaction (qRT-PCR) to prove knock out mutation. Transient reporter constructs were expressed according to the guidlines of the Tol2kit [22]. $25 \mathrm{ng}$ of DNA promotor plasmid in $0.5 \mu \mathrm{l}, 25 \mathrm{ng}$ of DNA reporter plasmid in $0.5 \mu \mathrm{l}$ and $1 \mu \mathrm{l}$ transposase Messenger ribonucleic acid (mRNA), diluted in $1 \mu \mathrm{l}$ water were prepared for injections. Small volumes of the $\mathrm{nl}$ scale were injected in fertilized embryos of the one-cell stage. Larvae were treated with PTU and imaged subsequently. Fish were raised to alduldhood to generate stable reporter lines. 
Table 10: PCR protocols mouse PCR

\begin{tabular}{lllll} 
& Cntnap1 WT (mm) & Cntnap1 $\mathrm{KO}(\mathrm{mm})$ & Caspr2 (mm) & $\mathrm{Mag}(\mathrm{mm})$ \\
\hline initiation & $94^{\circ} \mathrm{C}, 2 \mathrm{~min}$ & $94^{\circ} \mathrm{C}, 2 \mathrm{~min}$ & $94^{\circ} \mathrm{C}, 1 \mathrm{~min}$ & $94^{\circ} \mathrm{C}, 5 \mathrm{~min}$ \\
melting & $94^{\circ} \mathrm{C}, 30 \mathrm{sec}$ & $94^{\circ} \mathrm{C}, 20 \mathrm{sec}$ & $94^{\circ} \mathrm{C}, 30 \mathrm{sec}$ & $94^{\circ} \mathrm{C}, 1 \mathrm{~min}$ \\
annealing & $66^{\circ} \mathrm{C}, 30 \mathrm{sec}$ & $54^{\circ} \mathrm{C}, 20 \mathrm{sec}$ & $66^{\circ} \mathrm{C}, 30 \mathrm{sec}$ & $64^{\circ} \mathrm{C}, 1 \mathrm{~min}$ \\
elongation & $72^{\circ} \mathrm{C}, 35 \mathrm{sec}$ & $72^{\circ} \mathrm{C}, 30 \mathrm{sec}$ & $72^{\circ} \mathrm{C}, 1 \mathrm{~min}$ & $72^{\circ} \mathrm{C}, 30 \mathrm{sec}$ \\
repetitions & $30 \mathrm{x}$ & $30 \mathrm{x}$ & $30 \mathrm{x}$ & $35 \mathrm{x}$ \\
final elongation & $72^{\circ} \mathrm{C}, 3 \mathrm{~min}$ & $72^{\circ} \mathrm{C}, 5 \mathrm{~min}$ & $72^{\circ} \mathrm{C}, 5 \mathrm{~min}$ & $72^{\circ} \mathrm{C}, 5 \mathrm{~min}$ \\
end & $15^{\circ} \mathrm{C}$, hold & $15^{\circ} \mathrm{C}$, hold & $15^{\circ} \mathrm{C}$, hold & $15^{\circ} \mathrm{C}$, hold
\end{tabular}

Table 11: PCR protocols fish PCR

\begin{tabular}{llll} 
& cntnap1 & mag & nfascb \\
\hline initiation & $94^{\circ} \mathrm{C}, 2 \mathrm{~min}$ & $95^{\circ} \mathrm{C}, 2 \mathrm{~min}$ & $94^{\circ} \mathrm{C}, 1 \mathrm{~min}$ \\
melting & $94^{\circ} \mathrm{C}, 30 \mathrm{sec}$ & $95^{\circ} \mathrm{C}, 30 \mathrm{sec}$ & $94^{\circ} \mathrm{C}, 30 \mathrm{sec}$ \\
annealing & $66^{\circ} \mathrm{C}, 30 \mathrm{sec}$ & $60^{\circ} \mathrm{C}, 30 \mathrm{sec}$ & $66^{\circ} \mathrm{C}, 30 \mathrm{sec}$ \\
elongation & $72^{\circ} \mathrm{C}, 35 \mathrm{sec}$ & $72^{\circ} \mathrm{C}, 45 \mathrm{sec}$ & $72^{\circ} \mathrm{C}, 1 \mathrm{~min}$ \\
repetitions & $30 \mathrm{x}$ & $30 \mathrm{x}$ & $30 \mathrm{x}$ \\
final elongation & $72^{\circ} \mathrm{C}, 3 \mathrm{~min}$ & $72^{\circ} \mathrm{C}, 5 \mathrm{~min}$ & $72^{\circ} \mathrm{C}, 5 \mathrm{~min}$ \\
end & $15^{\circ} \mathrm{C}$, hold & $15^{\circ} \mathrm{C}$, hold & $15^{\circ} \mathrm{C}$, hold
\end{tabular}


Table 12: PCR mix mouse genotyping

\begin{tabular}{lllll} 
& Cntnap1 WT & Cntnap1 KO & Cntnap2 & Mag \\
\hline Go Taq Buffer + loading dye $(5 \mathrm{x})$ & $4 \mu \mathrm{l}$ & $4 \mu \mathrm{l}$ & $4 \mu \mathrm{l}$ & $4 \mu \mathrm{l}$ \\
primer for $(10 \mu \mathrm{M})$ & $0.5 \mu \mathrm{l}(34154)$ & $0.5 \mu \mathrm{l}(34156)$ & $0.5 \mu \mathrm{l}(34158)$ & $0.5 \mu \mathrm{l}(1864)$ \\
primer rev $(10 \mu \mathrm{M})$ & $0.5 \mu \mathrm{l}(34155)$ & $0.5 \mu \mathrm{l}(34157)$ & $0.5 \mu \mathrm{l}(34159)$ & $0.5 \mu \mathrm{l}(7650)$ \\
primer rev II $(10 \mu \mathrm{M})$ & - & - & $0.5 \mu \mathrm{l}(34160)$ & $0.5 \mu \mathrm{l}(7649)$ \\
$\mathrm{MgCl}_{2}$ & $1 \mu \mathrm{l}$ & $1 \mu \mathrm{l}$ & $1 \mu \mathrm{l}$ & $1 \mu \mathrm{l}$ \\
$\mathrm{dNTP}_{\text {mix }}$ & $1 \mu \mathrm{l}$ & $1 \mu \mathrm{l}$ & $1 \mu \mathrm{l}$ & $1 \mu \mathrm{l}$ \\
GoTaq & $0.1 \mu \mathrm{l}$ & $0.1 \mu \mathrm{l}$ & $0.1 \mu \mathrm{l}$ & $0.1 \mu \mathrm{l}$ \\
\hline DNA & $1 \mu \mathrm{l}$ & $1 \mu \mathrm{l}$ & $1 \mu \mathrm{l}$ & $1 \mu \mathrm{l}$
\end{tabular}

Table 13: PCR mix fish genotyping

\begin{tabular}{llll} 
& cntnap1 & mag & nfascb \\
\hline PCR mix & $15 \mu \mathrm{l}$ & $15 \mu \mathrm{l}$ & $15 \mu \mathrm{l}$ \\
primer for $(10 \mu \mathrm{M})$ & $0.5 \mu \mathrm{l}$ cntnap for $(\mathrm{dr})$ & $0.5 \mu \mathrm{l} \mathrm{mag} \mathrm{for}(\mathrm{dr})$ & $0.5 \mu \mathrm{l}$ nfascb_Ex\#3 for \\
primer rev $(10 \mu \mathrm{M})$ & $0.5 \mu \mathrm{l}$ cntnap rev $(\mathrm{dr})$ & $0.5 \mu \mathrm{l}$ mag rev $(\mathrm{dr})$ & $0.5 \mu \mathrm{l}$ nfascb_Ex\#3 for \\
GoTaq & $0.2 \mu \mathrm{l}$ & $0.2 \mu \mathrm{l}$ & $0.2 \mu \mathrm{l}$ \\
\hline DNA & $2 \mu \mathrm{l}$ & $2 \mu \mathrm{l}$ &
\end{tabular}

Table 14: PCR digestion

\begin{tabular}{ll} 
cut smart buffer $(10 \mathrm{x})$ & $1.3 \mu \mathrm{l}$ \\
restriction enzyme & $0.5 \mu \mathrm{l}$ \\
water & $1.2 \mu \mathrm{l}$ \\
\hline PCR product & $10 \mu \mathrm{l}$
\end{tabular}


Table 15: Mouse genotyping primer

\begin{tabular}{llll} 
number & gene & sequence & expected bands \\
\hline 34154 & Cntnap1 wt fov & GAGAGGGAAGGGTGGATAAGGAC & WT: $300 \mathrm{bp}$ \\
34155 & Cntnap1 wt rev & ATTGCGGAGCGCTGGGGAGAGG & \\
\hline 34156 & Cntnap1 ko fov & ATTTCCCAACGGCAGGTT & KO: $446 \mathrm{bp}$ \\
34157 & Cntnap1 ko rev & TCGCCTTCTTGACGAGTTC & \\
\hline 34158 & Cntnap2 rev & TCAGAGTTGATACCCGAGCGCC & WT: $400 \mathrm{bp}$ \\
34159 & Cntnap2 fov I & TGCTGCTGCCAGCCCAGGAACTGG & KO: $1000 \mathrm{bp}$ \\
34160 & Cntnap2 fov II & TTGGGTGGAGAGGCTATTCGGCTATG & \\
\hline 1864 & Mag fov & TTGGCGGCGAATGGGCTGAC & WT: $250 \mathrm{bp}$ \\
7650 & Mag rev I & ACGGCAGGGAATGGAGACAC & KO: $600 \mathrm{bp}$ \\
7649 & Mag rev II & ACCCTGCCGCTGTTTTGGAT &
\end{tabular}

Table 16: Zebrafish genotyping primer

\begin{tabular}{lll} 
name & sequence & restriction enzyme \\
\hline cntnap1 for & CAAATACATGGTGCTGTACG & NcoI-HF \\
cntnap1 rev & GGTCTATGCTTACAATGTTGG & \\
\hline cntn1b for & CGTCTTTAAATTTTACCTTAAGTGCC & AciI \\
cntn1b rev & TGCACTTTAACACAGATTAATGGAA & \\
\hline mag for & CTCTTTCTCTAAACAGATGCAAGC & HindIII-HF \\
mag rev & CGACAGAATTTTCATTGCTGG & \\
\hline nfascb_Ex\#3 for & AAAGAAGATCGCAATGGATGAT & AciI \\
nfascb_Ex\#3 rev & GGGCTCTTATCGTGTTTGTTTC &
\end{tabular}


Table 17: gRNAs used to generate zebrafish mutant lines

\begin{tabular}{llll} 
gene & location & sequence & source \\
\hline cntnap1 Exon 3 & TTCTCAGTATTCCAGCCCAT & $\begin{array}{l}\text { generated by Martina Arends (master } \\
\text { thesis roject), maintained by Sebastian } \\
\end{array}$ \\
& & & Timmler \\
\hline cntn1b & Exon & \multirow{2}{*}{ TCTGGACACTGTGTACGCGG } & generated and maintained by Dr. Mi- \\
& & & nou Djannatian \\
\hline mag & Exon 5 & \multirow{2}{*}{ AAGGGACGGACCAAGCTTCT } & generated and maintained by Dr. Mi- \\
& & & nou Djannatian \\
\hline \multirow{2}{*}{ nfascb } & Exon 3 & \multirow{2}{*}{ GCTGCCCATCCTGTTAGAAGCGG } & $\begin{array}{l}\text { generated and maintained by Sebastian } \\
\text { Timmler }\end{array}$
\end{tabular}

\subsubsection{Imaging in zebrafish}

Zebrafish larvae at 3, 4, 5 and $10 \mathrm{dpf}$ were anesthetized with tricaine, placed on imaging dishes and embedded in $0.8 \%$ low melting point agarose. Fish were imaged with an Leica SP8 confocal laser scanning microscope with automated moving stage and climate chamber $\left(28^{\circ} \mathrm{C}\right)$. All mutants were generated in mbp:eGFP_caax fish. Therefore, they were scanned with the $488 \mathrm{~nm}$ laser. Transient injected constructs were carrying mcherry, which was excited with the $552 \mathrm{~nm}$ laser.

Single images for quantification of myelinated cell bodies, myelinated area, number of myelinated sheaths per oligodendrocyte and myelin sheath length were taken with a 40x water immersion objective (1.1, using water), hybrid detector in counting mode, line accumulation of 4 , at $3 \times$ zoom, $1248 \times 1248$ pixels and the pinhole at 0.8 airy units. Galvo flow and bidirectional scanning were used. 6 z-stack tiles $(z$-step $=0.33 \mu \mathrm{m})$ along the spinal cord were acquired and stitched immediately using the LAS X software. 
Live imaging over time for quantification of extension and retraction events were acquired with a 40x water immersion objective (1.1, using Zeiss Immersol W), hybrid detector in counting mode, line accumulation of 8 , at 3x zoom, $1248 \times 1248$ pixels and a pinhole at 1.2 airy units. Galvo flow and bidirectional scanning were used. 13 z-stack tiles (z-step $=1 \mu \mathrm{m}$ ) along the spinal cord were taken every $30 \mathrm{~min}$. A transmitted light channel was taken simultaneously to surveil the health status. Images were stitched using the LAS X software.

\subsubsection{Electron Microscopy - conventional fixation of mouse tissue}

Mice were decapitated at the age of 12 and 21 days postnatal. Optic nerves were removed immediately and transferred to Karlsson-Schulz-solution (4\%PFA, $1 \%$ glutaraldehyde, in $0.2 \mathrm{M}$ phosphate buffer, $\mathrm{pH}=7.3$ ) for conventional fixation, the tail was clipped for re-genotyping. After 4 days of fixation at $4{ }^{\circ} \mathrm{C}$, optic nerves were wrapped in filter tissue to infuse them with osmium tetroxide, dehydrate them and subsequently substitute the buffer by Epoxy resin (EPON), using an automated tissue processor (Lynx II, Electron Microscopy Sciences) according to the protocol in Tab.18 (Möbius et al [81]). Infiltrated tissue samples were unwrapped, transferred to templates and molded into EPON. EPON blocks were baked for $24 \mathrm{~h}$ at $60^{\circ} \mathrm{C}$. Blocks were trimmed using a rotating diamond saw and ultrathin sections were cut with a diamond knife (DiATOME) at a Leica Microtome. 
Table 18: Tissue Processing Protocol

\begin{tabular}{lll}
\hline Time & Temperature ${ }^{\circ} \mathrm{C}$ & Action \\
\hline $3 \times 10$ min & 4 & wash with phosphate buffer \\
$4 \mathrm{~h}$ & 4 & incubation in $2 \% \mathrm{OsO}_{4}$ \\
$3 \times 10 \mathrm{~min}$ & 4 & wash with phosphate buffer \\
$2 \times 10 \mathrm{~min}$ & 4 & incubation in $30 \%$ ethanol \\
$2 \times 10 \mathrm{~min}$ & 4 & incubation in $50 \%$ ethanol \\
$2 \times 10 \mathrm{~min}$ & 4 & incubation in $70 \%$ ethanol \\
$2 \times 10 \mathrm{~min}$ & 4 & incubation in $90 \%$ ethanol \\
$4 \times 10 \mathrm{~min}$ & 4 & incubation in $100 \%$ ethanol \\
$3 \times 10 \mathrm{~min}$ & 21 & incubation in propylenoxide \\
$1 \mathrm{~h}$ & 21 & infiltration in propylenoxide:Epon mix $2: 1$ \\
$1 \mathrm{~h}$ & 21 & infiltration in propylenoxide:Epon mix $1: 1$ \\
$12 \mathrm{~h}$ & 21 & infiltration in propylenoxide:Epon mix $1: 2$ \\
$4 \mathrm{~h}$ & 21 & infiltration in Epon \\
\hline
\end{tabular}

\subsubsection{Electron Microscopy - fixation of mouse tissue by high pressure freezing}

Mice were euthanized by cervical dislocation and decapitated at the age of 12 and 21 days postnatal. Optic nerves were removed immediately and cryofixed by high pressure freezing in a HPM100 (Leica) within 5 minutes post decapitation, using polyvinylpyrrolidone as a filler. Samples were constantly kept under liquid nitrogen to prevent thawing and formation of ice crystals. Freeze substitution was performed by Marie-Theres Weil in a Leica AFS II at -90 ${ }^{\circ} \mathrm{C}$ and samples were embedded in EPON according to the tannic acid-OsO $\mathrm{O}_{4}$ protocol (Möbius et al.) [81]. Tissue samples were then transferred to gelatine capsules, molded in Epon and incubated for $24 \mathrm{~h}$ at $60^{\circ} \mathrm{C}$. 


\subsubsection{Electron Microscopy - conventional fixation of fish tissue}

Fixation was conducted as described before [168]. Zebrafish of $10 \mathrm{dpf}$ were anesthetized with tricaine and decapitated under a stereoscope. The head was used for DNA extraction and genotyping. The body of the fish was immediately (within $5 \mathrm{~min}$ ) transferred to freshly prepared fixative (4\% PFA, $1 \%$ glutaraldehyde, in $0.1 \mathrm{M}$ cacodyolate buffer) and kept on ice. The fish was microwaved 2 times at $100 \mathrm{~W}$ for $1 \mathrm{~min}(1 \mathrm{~min}$ cooling break each) and 5 times at $450 \mathrm{~W}$ for $30 \mathrm{~s}$ (30 s cooling break each). Fixative was exchanged and the fish was stored at least for 3 days, followed by postfixation in $2 \%$ osmium tetroxide in $0.05 \mathrm{M}$ imidazole and $0.1 \mathrm{M}$ sodium cacodylate and further contrasting in $1 \%$ tannic acid, saturated uranyl acetate, dehydrated into $100 \%$ aceton and embedded in EPON resin. After ultrathin sectioning the grids (Leica UC7 ultramicrotome) were contrasted by $1 \%$ uranyl acetate and Ultrostain (Leica). Images were acquired with a JEOL JEM1400 plus TEM equipped with a Ruby 8Mpx CCD camera. Data analysis was carried out using Fiji.

\subsubsection{Electron Microscopy - sectioning}

Ultrathin $(50 \mathrm{~nm})$ cross and longitudinal sections were generated using an Leica Ultracut $\mathrm{S}$ ultramicrotome with a Diatome Ultra $35^{\circ}$ diamond knife and transferred to 100 mesh hexagonal Formvar-coated EM copper grids. Grids were covered with Formvar by placing them on a floating film according to Peters and Pierson [167]. Samples were contrasted by placing the grids on droplets of $4 \%$ uranyl acetate in water for 30 min and washed six times in water droplets before drying with filter paper. Images were acquired with the LEO 912 Omega electron microscope (Zeiss) using an on-axis 2k CCD camera (TRS). 10 randomly picked cross section areas (225 $\mu \mathrm{m} 2$ each, 7.000x magnification) per animal were used to count double myelinated axons and 5 to quantify the ratio of myelinated versus unmyelinated axons. Overgrown nodes in longitudinal sections were counted in the area of one grid hexagon $(26.000 \mu \mathrm{m} 2)$ per animal. The area was imaged at 3000x magnification and images were stitched together. 


\subsubsection{Electron Microscopy - FIB-SEM}

Samples were prepared by high pressure freezing and embedded in EPON according to Möbius et al. [81] FIB-SEM was performed by Marie-Theres Weil at the MPI for experimental Medicine, using a Zeiss Crossbeam FIB-SEM. The images were acquired with a $3 \mathrm{~nm}$ pixel size in $\mathrm{x} / \mathrm{y}$ and a z-depth of $50 \mathrm{~nm}$. An area of $13 \mu \mathrm{m}$ x $6 \mu \mathrm{m}$ was scanned. 3D reconstruction was performed by Sebastian Timmler using the MIB software.

\subsubsection{Electron Microscopy - ATUM}

Fixed mouse optic nerve samples were en bloc stained by a standard rOTO protocol (Tapia et al.) [169] in a sequence of reduced $2 \%$ osmium tetroxide in $1.5 \%$ potassium ferrocyanide in 0.1 M cacodylate buffer $\mathrm{pH} 7.4,1 \%$ aqueous thiocarbohydrazide (TCH) and 2\% aqueous osmium tetroxide including washing steps. After an over night incubation in $1 \%$ uranylacetate at $4^{\circ} \mathrm{C}$, samples were contrasted in $0.0665 \%$ lead aspartate, dehydrated and infiltrated with Epon. The blocks were trimmed by $200 \mu \mathrm{m}$ to expose a rectangular tissue block using a TRIM90 knife (Diatome) on a Powertome ultramicrotome (RMC). Consecutive sections were taken with a diamond ultra knife (Diatome) at $150 \mathrm{~nm}$ thickness and collected on plasma-treated, carbon-coated Kapton tape (kindly provided by Richard Schalek, Jeff Lichtman, Harvard) (Kasthuri et al.) [170]. Kapton strips with tissue sections were assembled onto carbon tape (Science Services), mounted onto a 4-inch silicon wafer (Siegert Wafer) and grounded with adhesive carbon tape strips. Section images were acquired on a Crossbeam Gemini 340 SEM (Zeiss) in backscatter mode at $8 \mathrm{keV}$ (high gain) at $7.0 \mathrm{~mm}$ WD and 60 ţm aperture. In ATLAS5 Array Tomography (Fibics, Ottawa, Canada) the entire wafer was imaged at $6000 \mathrm{~nm} /$ pixel followed by mapping and medium resolution (100 nm/pixel) imaging of individual tissue sections. A region of interest comprising $55 \times 55 \mu \mathrm{m}$ on 367 sections on two wafers was automatically acquired at $4 \mathrm{~nm} /$ pixel. The images were aligned using Fiji TrakEM2 (Cardona et al.) [171]. The reconstruction was done using VAST (Kasthuri et al.) [170]. Image analysis was done in Fiji. 


\subsubsection{Statistics}

Statistics were performed in R. All samples were tested for normality and equal variances. If acceptable, one-way ANOVA was performed, followed by pairwise student's t-test, using Bonferroni correction. If ANOVA was not applicable, pairwise Wilcoxon-signed-rank test was performed, using Bonferroni or Dunn's correction. Data in the text is presented as mean $\pm \mathrm{SD}$. 


\section{Results}

\subsection{Localization of the paranodal adhesion proteins in early development}

\subsubsection{Mouse}

The function of the paranodal adhesion complex during node formation is well understood, whereas its role in the dynamics of the sheath forming remains unclear. We analyzed the localization of Caspr during growth of a myelin sheath in mice of different age. We stained cervicothoracial spinal cord sections of PFA-fixed wildtype mice for Caspr (1:1000) and AnkG (1:250). During node formation, AnkG signal shifts from the paranodal region in glia to a neuronal nodal region. Together with the distance between the Caspr positive heminodes, the AnkG shift was used to define 5 stages of node maturation: [P] paranodal, [PN] paranodal+nodal, [PNP] paranodal+nodal peak, $[\mathrm{N}]$ nodal, $[\mathrm{O}]$ other structure. The number of each maturation stage present in samples of P4, P7, P12 and P20 animals was counted. At least $80 \%$ of the nodes could be assinged to a distinct category ([P], [PN], [PNP] or $[\mathrm{N}])$. At P4, stage $[\mathrm{P}]$ and $[\mathrm{PN}]$ prevail, whereas during development, AnkG localization changes and the nodal gap closes (category $[\mathrm{N}]$ is predominant) (see Fig.4). We measured the distance between two apposing Caspr signals referring to a nodal gap and the length of the paranodal Caspr signal. During development the average distance between the Caspr signals decreases (P4: 10.4 $\pm 7.1 \mu \mathrm{m}, \mathrm{P} 7: 6.0 \pm 4.1$ $\mu \mathrm{m})$, the nodal gaps are closing until most of them reach their final size (P7: $2.8 \pm 1.6 \mu \mathrm{m}, \mathrm{P} 12$ : $3.0 \pm 1.3 \mu \mathrm{m})$. At the same time, the length of the Caspr signal was not changing with development, indicating that the paranodal localization of Caspr was not altered at this stage. Caspr localization at earlier stages of initiation or sheath growth could not be detected. The spirally positioning of Caspr at the paranodes was confirmed by high-resolution expansion microscopy (see Fig.4). 

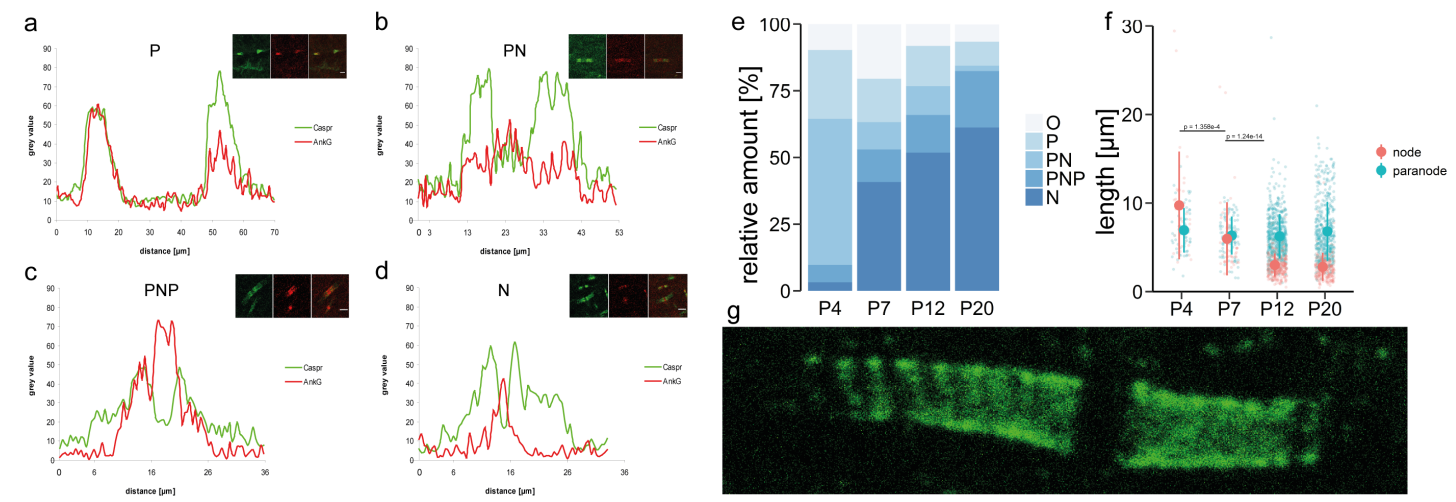

Figure 4: Stages of node development indicated by Caspr and AnkG stainings

Representative images and measured spectra (a) paranodal [P] (b) paranodal+nodal $[\mathrm{PN}](\mathrm{c})$ paranodal+nodal peak $[\mathrm{PNP}](\mathrm{d})$ nodal $[\mathrm{N}]$, scalebars $=10 \mu \mathrm{m}(\mathrm{e})$ Stages of node development at P4, P7, P12 and P21 (f) nodal and paranodal distance in spinal cord sections at P4, P7, P12 and P21 (g) Caspr staining in paranodes, aquired using expansion microscopy, scale can not be set due to unknown expansion factor.

\subsubsection{Fish}

In mammals, the paranodal adhesion complex consists of the intercellular binding partners Cntn1 and Nfasc155, connected to the axonal cytoskeleton by the transmembrane scaffold Caspr. Due to genome duplications in zebrafish, Cntnl and Nfasc split up to contactinl a (cntnla), cntnlb, neurofascin a (nfasca) and neurofascin b (nfascb), whereas caspr has no paralogue (see chapter 1.1). Therefore, at first it was nescessary to identify the teleost paralogues with equivalent functions. To find the paralogues that localize to the paranodal junctions, we co-injected the plasmids UAS:cntn1a_eGFP, UAS:cntn1b_eGFP together with huc:GAL4 in Tg(Sox10:mRFP). Cntn1a showed nodal localization as previously demonstrated[172], whereas cntn1b was delivered to the paranodes. UAS:Nfascb_eGFP was injected together with the glial promoter construct sox10:GAL4 and showed paranodal localization (see App.Fig.31). Neuronal expression of UAS:Caspr_YFP demonstrated export to the paranodes, indicating that the fish paranodal adhesion complex consists of cntn1b, nfascb and caspr. As in stainings of mouse tissue (see chapter 3.1.1), early engagement of caspr in sheath growth could not be shown. 


\subsection{Single depletion of internodal and paranodal adhesion molecules}

\subsubsection{Disruption of the paranodal adhesion complex leads to cell body myelination in zebrafish}

Functions of a protein can be examined by studying gene knock out mutant model systems and the most suitable way to track myelination in vivo is using zebrafish reporter lines. Therefore, we combined both and used Crispr/Cas9 mediated genome editing to deplete caspr, cntnlb, and $n f a s c b$ in $\operatorname{Tg}(\mathrm{mbp}:$ eGFP-CAAX) fish. Injected founder animals were bred until homozygous F2 offspring could be generated from heterozygous adults. Successful gene inactivation was proven by qRT-PCR (see App.Fig.30). Homozygous cntn $1 b^{-/-}$, cntnap $1^{-/-}$and nfascb ${ }^{-/-}$mutants were smaller in size, showed ataxic swimming behavior, developed a curved spine and were not able to breed. Larvae were analyzed at 3, 4 and 10 dpf. All single mutant fish showed myelinated circular profiles (see Fig.5a). Coexpression of huc:mcherry in the same fish could prove that they resemble neuronal cell bodies (see Fig.5e). Myelinated cell bodies is a striking observation of mistargeted myelination. Thus, the number of myelinated cell bodies was counted in one hemisphere of the anterior third of the spinal cord. Numbers were normalized to the total area of mbp:eGFP-signal (the amount of myelin that was made) in the same field of view, to correct for possible hypomyelination (see Fig.5b). All three mutants showed values of about 50 at $3 \mathrm{dpf}\left(\right.$ cntnl $^{-1-}: 40.4 \pm 28.01 / 10000 \mu \mathrm{m}^{2}$, tnapl ${ }^{-/-}: 50.3 \pm 17.91 / 10000 \mu \mathrm{m}^{2}$, $\left.n f a s c b^{-1-}: 66.2 \pm 54.51 / 10000 \mu \mathrm{m}^{2}\right)$ going up to about 64 to 89 at $10 \mathrm{dpf}\left(\right.$ cntnlb $^{-1-}: 63.7 \pm 25.6$ $1 / 10000 \mu \mathrm{m}^{2}$, cntnap $^{-1-}: 88.5 \pm 16.21 / 10000 \mu \mathrm{m}^{2}$, nfasch $\left.{ }^{-/-}: 75.7 \pm 38.51 / 10000 \mu \mathrm{m}^{2}\right)$. Numbers in $\mathrm{cntnl}^{-/-}$mutants stayed lower at $4 \mathrm{dpf}\left(11.3 \pm 13.11 / 10000 \mu \mathrm{m}^{2}\right)$ but catched up at $10 \mathrm{dpf}$. In all mutant fish, the number of myelinated cell bodies increased with development. In contrast, wildtype fish exhibited a low number of myelinated cell bodies at $3 \mathrm{dpf}\left(8.8 \pm 7.71 / 10000 \mu \mathrm{m}^{2}\right)$ that completely disappeared with maturation, already at $4 \mathrm{dpf}\left(0.73 \pm 1.51 / 10000 \mu \mathrm{m}^{2}\right)(\mathrm{see}$ Fig.5c). Accumulating myelinated cell bodies in mutants could be a result of failing correction by process retraction. We assessed that by in vivo timelapse recordings. We measured the 


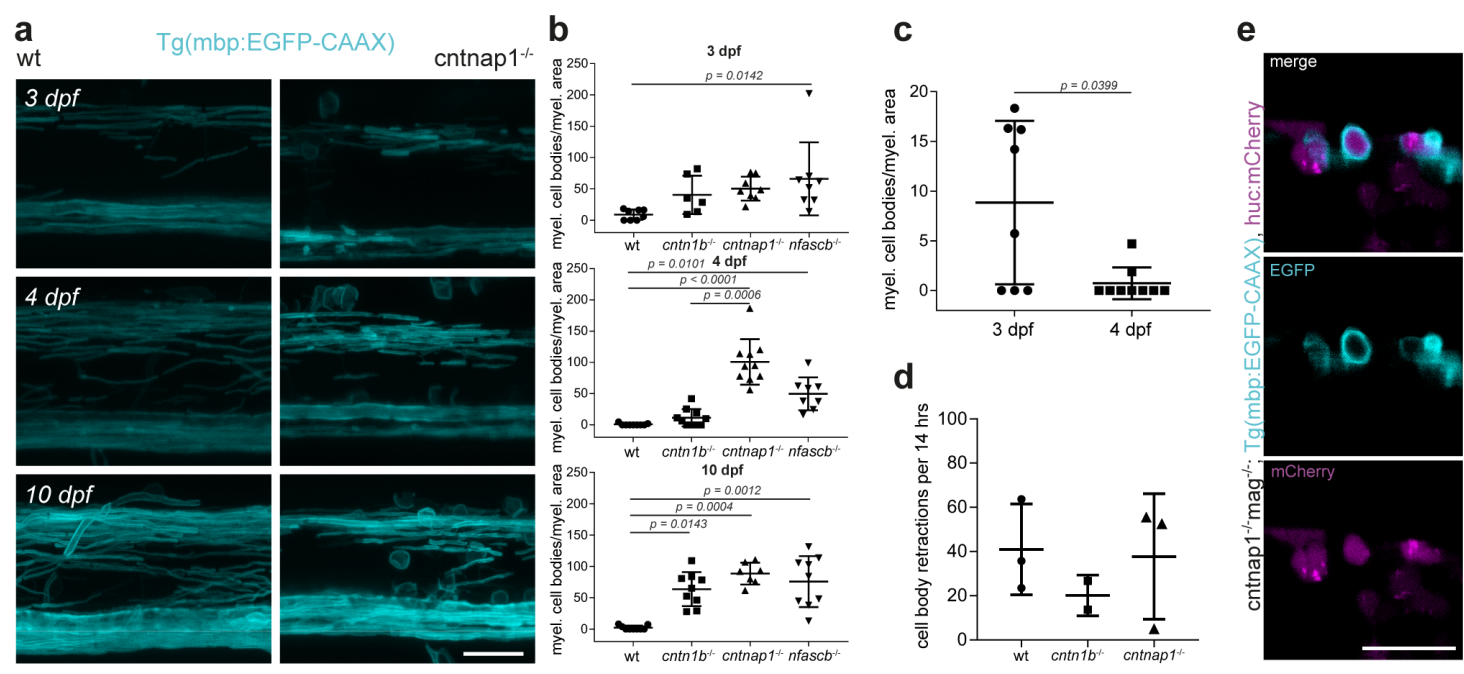

Figure 5: Myelinated cell bodies in paranodal protein mutant fish. (a) Micrographs of wildtype controls and mutant fish generated in Tg(mbp:eGFP-CAAX), scale bar $=20$ $\mu \mathrm{m}$ (b) Quantification of myelinated cell bodies normalized to the myelinated area at 3, 4 and $10 \mathrm{dpf}$ (c) Quantification of myelinated cell bodies in wildtype animals at 3 and $4 \mathrm{dpf}$ (d) Quantification of retractions from cell bodies during $14 \mathrm{hrs}$, acquired by live imaging (e) Representative micrograph of a myelinated cell body with transient expression of mbp:mcherry in $\mathrm{Tg}(\mathrm{mbp}: \mathrm{eGFP}-\mathrm{CAAX})$ cntnap $^{-/-} \mathrm{mag}^{-{ }_{-}}$, it proofs that circular profiles are neuronal cell bodies, scalebar $=20 \mu \mathrm{m}$; in all quantifications we performed ANOVA, followed by pairwise t-test with p-value adjustment by Bonferroni method.

rate of glial retractions from cell bodies between 3 and $4 \mathrm{dpf}$ in $c n t n 1 b^{-/-}$, cntnap $1^{-/-}$mutants and wildtype controls (see Fig.5d). We found, that oligodendrocytes of mutant and wildtype fish during 14 hours, retracted from 20 to 40 cell bodies with no significant difference. Correction of mistargeted myelin seems not to be impaired in single mutants.

\subsubsection{Mag regulates lateral growth of zebrafish myelin sheaths}

Mag is an important internodal adhesion protein that binds to ganglioside on the axonal membrane and has effects on the extend of myelination. Therefore, we depleted mag in a zebrafish reporter line. Mutants lacking mag were generated in Tg(mbp:eGFP-CAAX) using Crispr/Cas9 mediated genome editing and exhibited no visible phenotype. Successful gene inactivation was proven by qRT-PCR (see App. Fig30). Homozygous mag mutants were of similar size, showed 
normal swimming behavior and could breed. Similar as in wildtype controls, neuronal cell body myelination was rarely observed in 3, 4 and $11 \mathrm{dpf}$ mag mutants. Myelinated area of the upper third of the dorsal spinal cord (1 hemisphere) was measured from tresholded eGFP signal of maximum z projections (see Fig6). At $10 \mathrm{dpf}$, homozygous $c n t n 1 b^{-/-}\left(7275 \pm 807 \mu \mathrm{m}^{2}\right)$, cntnap $^{\text {-- }^{-}}\left(6731 \pm 1001 \mu \mathrm{m}^{2}\right)$ or $n f a s c b^{-I_{-}}\left(6932 \pm 1021 \mu \mathrm{m}^{2}\right)$ mutants exhibit no alteration in the myelinated area compared to control fish $\left(7991 \pm 696 \mu \mathrm{m}^{2}\right)$. Mutants lacking mag showed a reduced myelinated area $\left(6442 \pm 673 \mu \mathrm{m}^{2}\right)$. The number of myelinated axons (counted in conventional fixed TEM micrographs at $10 \mathrm{dpf})$ was reduced in $\mathrm{mag}^{-/-}(18.8 \pm 9.5)$ and $\mathrm{cntn}^{-b^{-/}}$ mutant fish (21.3 \pm 1.6$)$ compared to wildtype controls (38.8 \pm 4.9$)$ (see Fig.6h-i). This discrepancy can be cleared up by looking at the individual sheath length. In 4 dpf fish, sheath length was reduced in mutants of mag $(12.7 \pm 6.6 \mu \mathrm{m}), n f a s c b(11.8 \pm 6.0 \mu \mathrm{m})$ and cntnapl $(11.7 \pm 6.3 \mu \mathrm{m})$ compared to wildtype $(16.9 \pm 8.6 \mu \mathrm{m})$. At $10 \mathrm{dpf}$ the average sheath length of all single mutants increased except for the $\mathrm{mag}^{-{ }^{--}}$mutant $(14.2 \pm 8.4 \mu \mathrm{m})$. Mutants lacking cntnapl $(19.6 \pm 10.5$ $\mu \mathrm{m})$ or $n f a s c b(17.3 \pm 11.5 \mu \mathrm{m})$ still showed shorter sheaths than wildtype controls $(24.3 \pm 10.5$

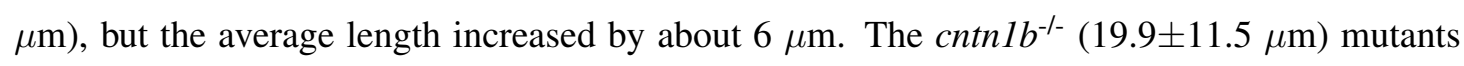
had slightly reduced sheath lengths. Shapiro-Wilk test for normal distribution and histogram analysis revealed, that the length of wildtype sheaths was almost normally distributed at both time-points, whereas all mutants showed a skewness towards shorter sheaths. The number of oligodendrocytes, counted at P4 (eGFP positive cell bodies connected to multiple eGFP positive myelin sheaths) was not altered in mag mutants, but slightly increased in cntnapl mutants (see Fig.6c). The average amount of sheaths (eGFP positive sheaths connected to a eGFP positive oligodendrocyte cell body) formed by an individual oligodendrocyte was lowered by all single mutations $\left(\right.$ cntnlb $^{--}: 17.0 \pm 4.1 ;$ cntnap $^{-/-}: 13.2 \pm 4.3 ;$ nfasch $^{-/-}: 16.2 \pm 3.0 ;$ mag $\left.^{-I_{-}}: 15.3 \pm 4.1\right)$ compared to wildtype (20.1 \pm 3.1$)$ (see Fig.6d-e). 

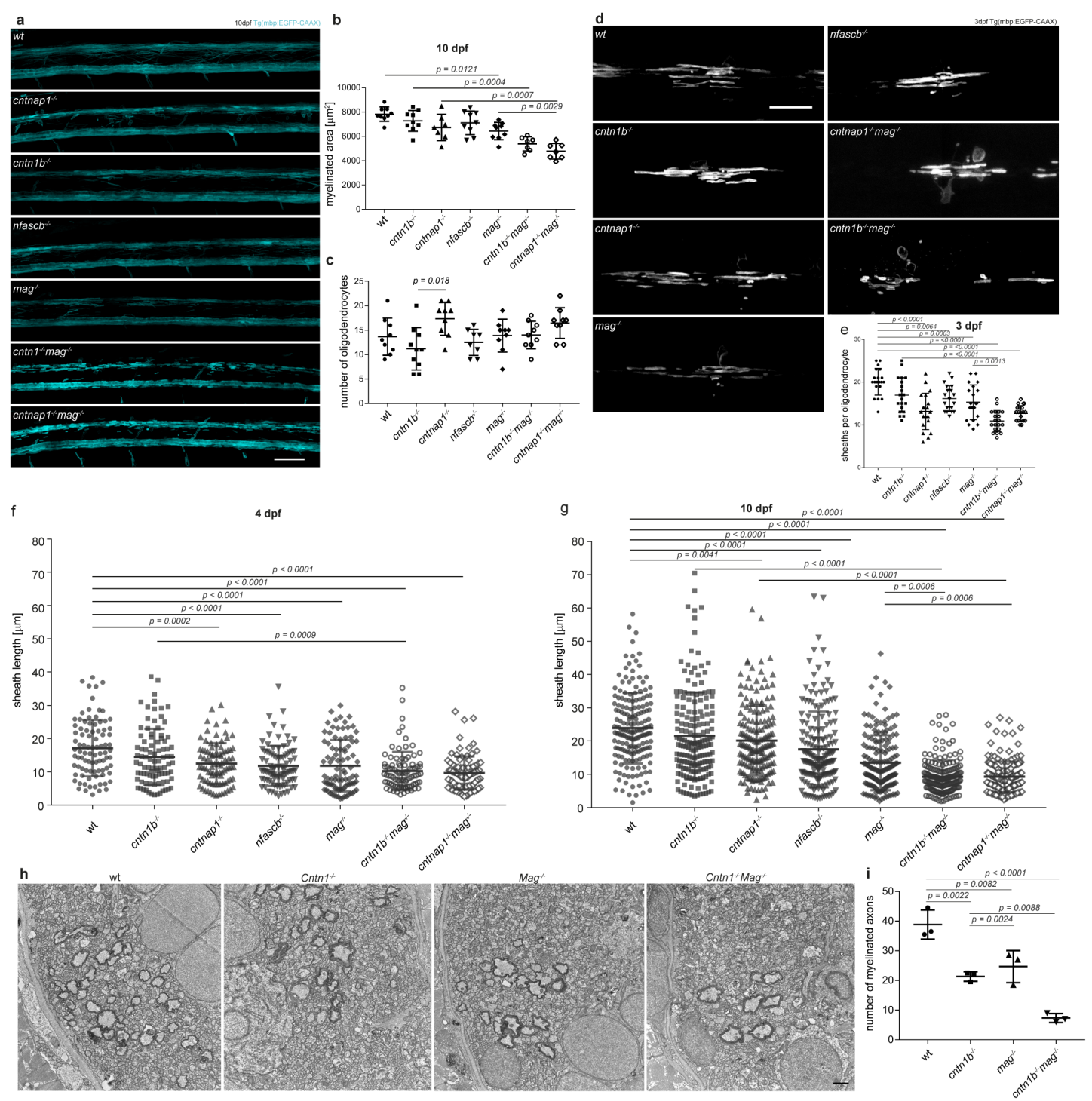

Figure 6: Myelin properties in single and double mutant fish. (a) Micrographs of wildtype controls and all mutant fish generated in Tg(mbp:eGFP_CAAX), scale bar $=50 \mu \mathrm{m}$ (b) Quantification of myelinated area from $10 \mathrm{dpf}$ dorsal spinal cord maximum projections, statistical test: Kruskal-Wallis, p-value adjustment by Dunn's method (c) Quantification of oligodendrocyte numbers in 4 dpf dorsal spinal cord (d)

Representative micrographs of individual oligodendrocytes in dorsal spinal cord, scalebar $=25 \mu \mathrm{m}$ (e) Quantification of sheaths per oligodendrocyte from $3 \mathrm{dpf}$ dorsal spinal cord, ANOVA, followed by pairwise t-test, p-value adjustment by Bonferroni method (f) Quantification of myelin sheath length in $4 \mathrm{dpf}$ dorsal spinal cord (g) Quantification of myelin sheath length in $10 \mathrm{dpf}$ dorsal spinal cord (h) Representative TEM micrographs of fish spinal cord at $10 \mathrm{dpf}$, scale bar $=500 \mathrm{~nm}$ (i) Quantification of myelinated axons in TEM micrographs; in all quantifications we performed ANOVA, followed by pairwise t-test with p-value adjustment by Bonferroni method . 

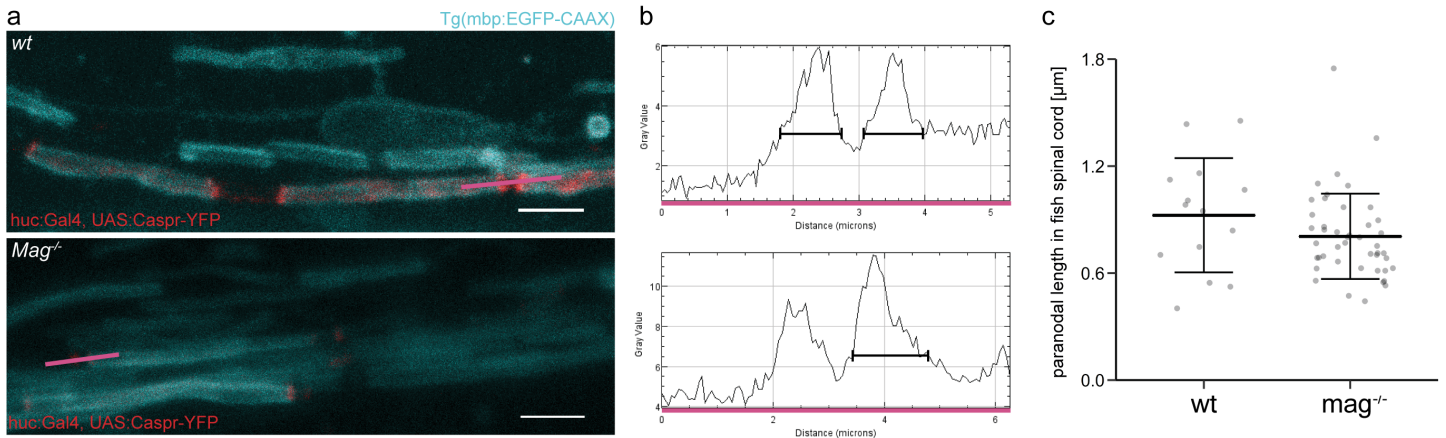

Figure 7: Transient expression of UAS:caspr-YFP under control of huc:Gal4 in

Tg(mbp:eGFP_CAAX) mag $^{-/-}$fish (a) Micrographs of caspr localization, pink lines indicate where the spectrum was measured (average of 10 parallel lines), scalebar $=5$ $\mu \mathrm{m}$ (b) Intensity spectra along the respective line, black lines indicate the measured distance (c) Quantification of individual paranodal lengths in two fish per genotype (Wilcoxon rank sum test: $\mathrm{p}=0.1646$ ).

\subsubsection{Caspr-localization is not impaired in Mag-depleted fish or mice}

Since adhesion provides structural guidance, the paranodal adhesion proteins could change their localization in the absence of internodal adhesion (mag). To assess this possibility, we injected a UAS:caspr-YFP construct together with huc:Gal4 in $\mathrm{mag}^{-/-} \mathrm{Tg}(\mathrm{mbp}: \mathrm{eGFP}-\mathrm{CAAX})$-reporter fish and measured the length of paranodal intensity signal corresponding to the caspr-YFP fusion protein. We were able to measure paranodal distances in two fish per genotype. There was no difference in paranodal length between $\mathrm{mag}^{-/-}$mutant $(0.81 \pm 0.2 \mu \mathrm{m})$ and wildtype fish $(0.92 \pm 0.3 \mu \mathrm{m})$ (see Fig7). No differing distribution patterns of caspr-YFP could be observed in $\mathrm{mag}^{-/-}$fish.

Longitudinal optic nerve cryo-sections $(50 \mu \mathrm{m})$ of P21 Mag deficient mice were stained for Caspr and pan-Nfasc. Distances of the paranodes were measured by the length of the Caspr signal. Nodal length was measured as the distance between to corresponding Caspr signals or the length of the pan-Nfasc signal. There was no difference in paranodal length between $\mathrm{Mag}^{-/-}$ mutant $(1.25 \pm 0.53 \mu \mathrm{m})$ and wildtype mice $(1.18 \pm 0.61 \mu \mathrm{m})$ (see Fig8). No differing distribution patterns of Caspr could be observed in $\mathrm{Mag}^{-/-}$mice. 

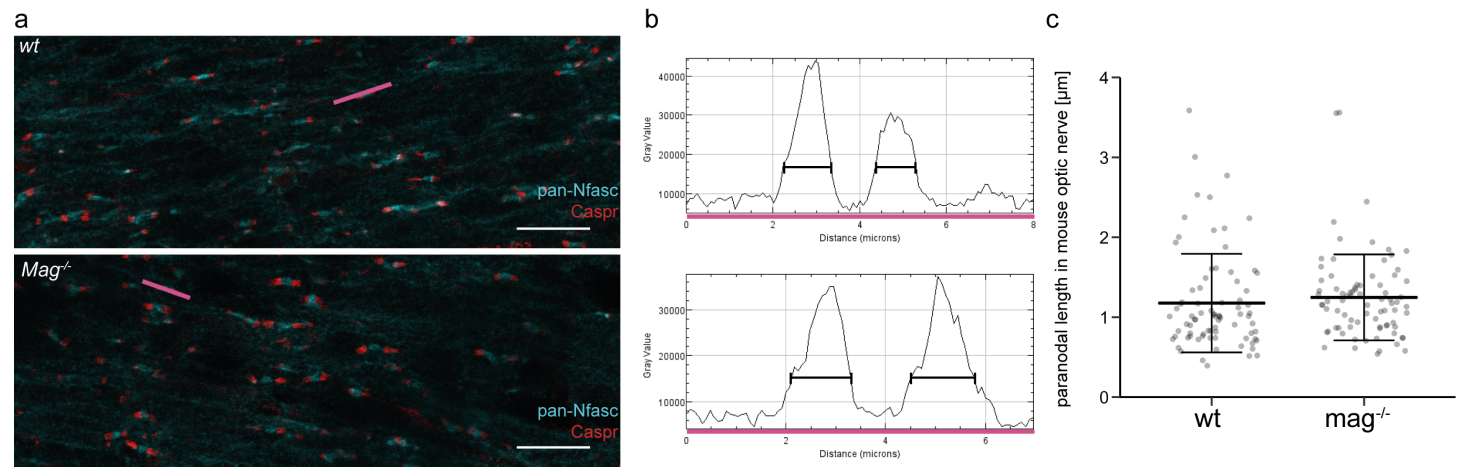

Figure 8: Immunohistochemistry approach to assess Caspr localization in Mag deficient mice (a) Micrographs of wildtype and $\mathrm{Mag}^{-/-}$mice, pink lines indicate where the spectrum was measured (average of 10 parallel lines), scalebar $=\mu \mathrm{m}$ (b) Intensity spectra along the respective line, black lines indicate the measured distance (c) Quantification of individual paranodal lengths in two mice per genotype (Wilcoxon rank sum test: $\mathrm{p}=0.05469$ ).

\subsection{Combined depletion of internodal and paranodal adhesion molecules causes defects in myelin lateral growth}

To address the different functions of the paranodal proteins, implicated by the results of chapter 3.2.2, we crossbred the single knock out mutants to generate combined mutants. Additional depletion of mag in cntnapl or cntnlb mutants caused spinal cord deformation, exacerbated ataxia and smaller body size than in the wildtype controls. Breeding the homozygous double mutants was not possible. Double homozygous animals were generated from breedings of

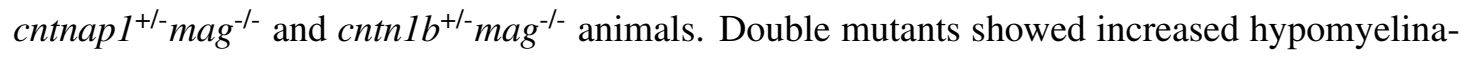
tion. At $10 \mathrm{dpf}$, the myelinated area of the upper third of the dorsal spinal cord (1 hemisphere) of double mutants was smaller (cntnap1 ${ }^{-/-}$mag $^{-/-}: 4779 \pm 597 \mu^{2}$ cntnl $^{-/-}$mag $^{-/-}: 5386 \pm 538$ $\mu \mathrm{m}^{2}$ ) than that of wildtype control $\left(7991 \pm 696 \mu \mathrm{m}^{2}\right)$ and single mutants (see chapter3.2.2). The number of myelinated axons (counted in conventional fixed TEM micrographs at $10 \mathrm{dpf}$ ) was markedly reduced in $\mathrm{cntn} \mathrm{b}^{-{ }_{-}} \mathrm{mag}^{-{ }_{-}}$mutants (7.3 \pm 1.5$)$ compared to single mutants and wildtype (see chapter3.2.2 and Fig.6h-i). Ectopically myelination of neuronal cell bodies was observed as in both paranodal single mutants (data not shown). Average sheath length was clearly reduced at $4 \mathrm{dpf}\left(\right.$ cntnap $^{-/}$mag $^{-/}: 9.6 \pm \mathrm{m} 5.0 \mu \mathrm{m}$, cntnlbmag: $\left.10.2 \pm 5.7 \mu \mathrm{m}\right)$ and did not improve at $10 \mathrm{dpf}$ 


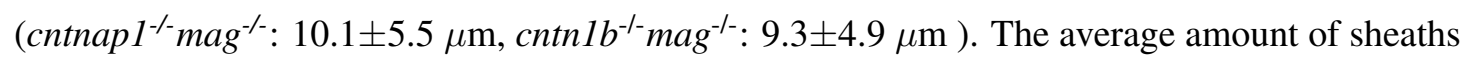
formed by an individual oligodendrocyte was lowered in $\mathrm{cntnl}^{-/-} \mathrm{mag}^{-/-}$mutants $(10.9 \pm 2.4)$ compared to wildtype and single mutants and in cntnap $1^{-/-}$mag $^{-/-}$mutants (12.7 \pm 1.8$)$ compared to wildtype animals (see Fig.6d-e).

We performed $16 \mathrm{~h}$ live-imaging experiments of the upper half of the spinal cord of wildtype and mutant fish ( $\mathrm{n}=3$ ). Movement of individual sheaths was tracked using 3D analysis software (bitplane IMARIS). Rates were calculated from the slope of the graph during active growing or retraction phase. The average extension rates were lowered in single mutants $\left(\mathrm{mag}^{-/-}: 0.79 \pm 0.2\right.$ $\mu \mathrm{m} / \mathrm{h},{\text { cntn } 1 b^{--}:}_{0} 0.63 \pm 0.25 \mu \mathrm{m} / \mathrm{h}$, cntnapl $\left.^{-/-}: 0.86 \pm 0.44 \mu \mathrm{m} / \mathrm{h}\right)$, compared to wildtype controls $(1.07 \pm 0.34 \mu \mathrm{m} / \mathrm{h})$ and again lower in combined double knock out animals (cntnl b $^{-/}$mag $^{-1-}$ : $0.47 \pm 0.35 \mu \mathrm{m} / \mathrm{h}$, cntnapl $^{-/-} \mathrm{mag}^{-/-}: 0.41 \pm 0.19 \mu \mathrm{m} / \mathrm{h}$ ), indicating additional physical impairment in lateral growth (see Fig.9 and Fig.11a). Similarly, average retraction rates were reduced in $\left(\right.$ mag $^{-/-}: 1.78 \pm 1.5 \mu \mathrm{m} / \mathrm{h}$, cntn $^{-b^{--}}: 2.07 \pm 1.55 \mu \mathrm{m} / \mathrm{h}$, cntnap $\left.^{-{ }^{--}}: 1.84 \pm 1.68 \mu \mathrm{m} / \mathrm{h}\right)$ and combined double knock out animals (cntn $1 b^{-/-} \mathrm{mag}^{-/-}: 0.51 \pm 0.28 \mu \mathrm{m} / \mathrm{h}$, cntnap $^{-I_{-}} \mathrm{mag}^{-{ }^{--}}: 0.54 \pm 0.28$ $\mu \mathrm{m} / \mathrm{h})$ compared to wildtype controls $(1.99 \pm 1.49 \mu \mathrm{m} / \mathrm{h})$, indicating physical impairment of retraction (see Fig.10 and Fig.11b). Retractions in either cntnap $^{-/-} \mathrm{mag}^{-/-}$or $\mathrm{cntn}^{-b^{-/}} \mathrm{mag}^{-{ }_{-}}$double mutants very often resulted in inflating myelin sheaths that subsequently disintegrated. A process we call blebbing of sheaths. The numbers of blebbing events were counted in the liveimaging dataset (see Fig.12).

A striking observation in both double mutants was sheaths that display a distinct step of intensity increase by $\sim 100 \%$. By co-expression of a mbp:mcherry construct, we could show, that these are multiply myelinated axons with sheaths belonging to independent oligodendrocytes (see Fig.13). We assumed that an intensity step of $100 \%$ is equivalent to a doubled myelin sheath and quantified them in $10 \mathrm{dpf}$ fish. Double intensity sheaths were not observed in wildtype animals $\left(0.4 \pm 0.61 / 10000 \mu \mathrm{m}^{2}\right)$ and occured in single mutants (cntnap $1^{--}: 28.2 \pm 11.81 / 10000 \mu \mathrm{m}$, cntnl $^{-/-}: 27.8 \pm 10.71 / 10000 \mu \mathrm{m}$, mag $\left.^{-/-}: 2.9 \pm 3.11 / 10000 \mu \mathrm{m}\right)$. 

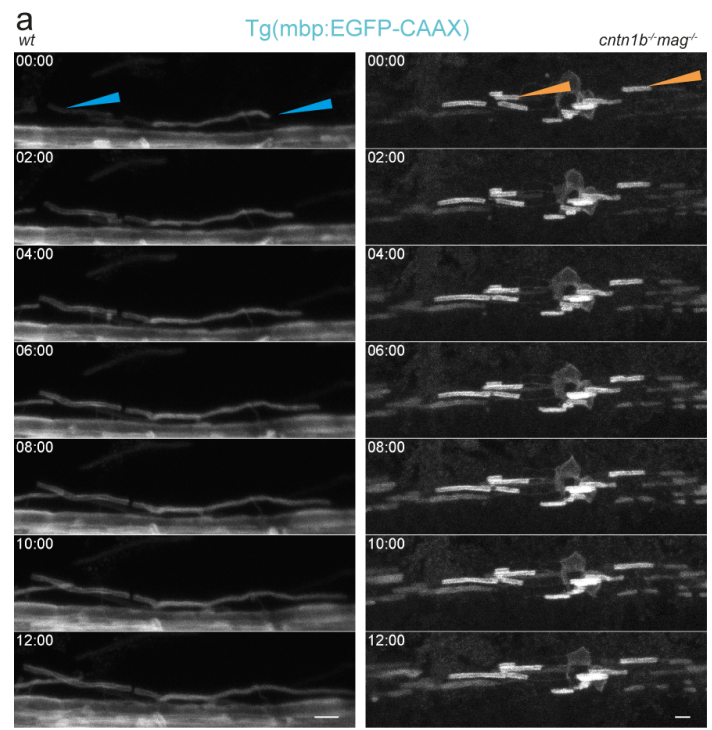

b
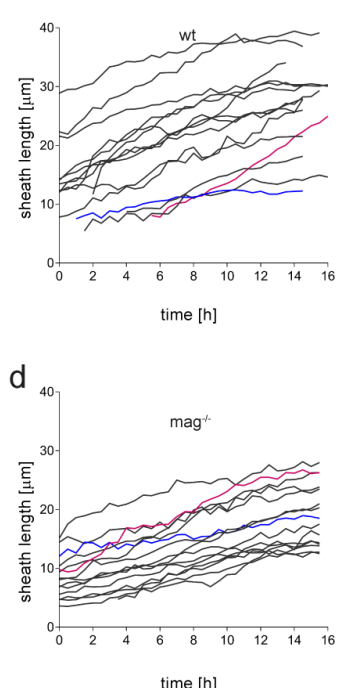

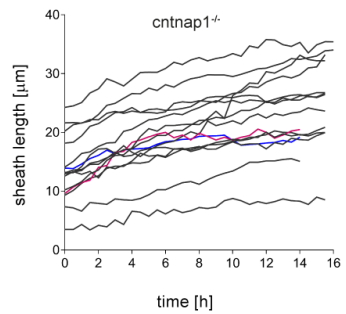

e

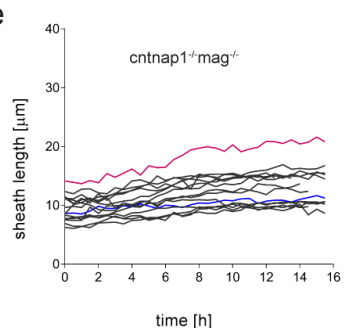

Figure 9: Extension of myelin sheaths in wildtype and mutant fish (a) Sequential frames of a live imaging experiment in wildtype and $\mathrm{cntn} 1 \mathrm{~b}^{-\mathrm{m}_{-}} \mathrm{mag}^{-{ }_{-}}$mutant, blue arrowheads indicating extending segments, orange arrowheads indicating slowly extending segments, scalebar $=5 \mu \mathrm{m}$ (b) Extension of single myelin sheaths during time in wildtyp animals, red indicates the fastest, blue the slowest sheath (c) Extension of single myelin sheaths during time in cntnap $^{-/-}$animals, red indicates the fastest, blue the slowest sheath (d) Extension of single myelin sheaths during time in $\mathrm{mag}^{-/-}$ animals, red indicates the fastest, blue the slowest sheath. (e) Extension of single myelin sheaths during time in cntnap $^{I_{-/}} \mathrm{mag}^{-/-}$animals, red indicates the fastest, blue the slowest sheath. 

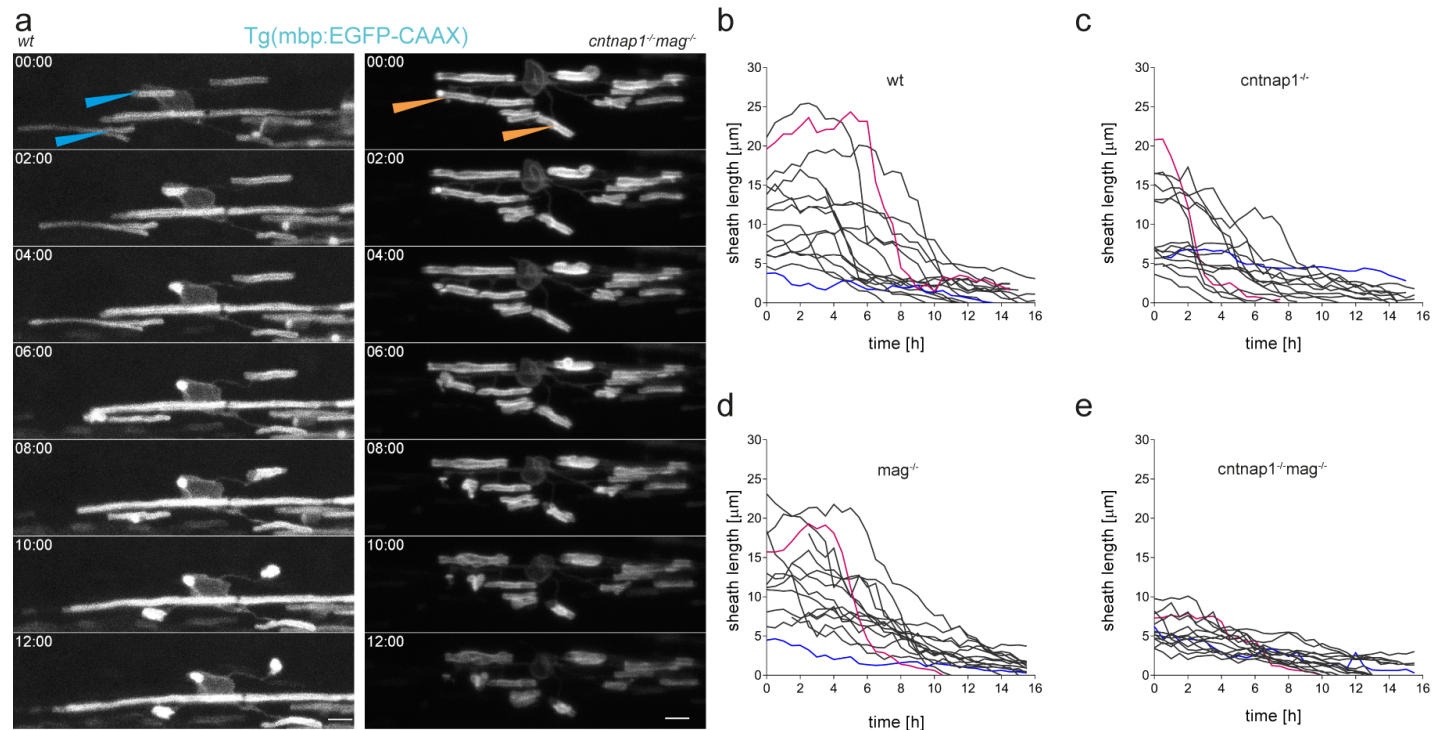

Figure 10: Retraction of myelin sheaths in wildtype and mutant fish (a) Sequential frames of a live imaging experiment in wildtype and cntnap $^{-I_{-}} \mathrm{mag}^{-{ }_{-}}$mutant, blue arrowheads indicating retracting segments, orange arrowheads indicating retracting segments that start to disintegrate and blebb, scalebar $=5 \mu \mathrm{m}$ (b) Retraction of single myelin sheaths during time in wildtyp animals, red indicates the fastest, blue the slowest sheath (c) Retraction of single myelin sheaths during time in cntnap $1^{-/}$ mutants, red indicates the fastest, blue the slowest sheath (d) Retraction of single myelin sheaths during time in $\mathrm{mag}^{-/-}$mutants, red indicates the fastest, blue the slowest sheath (e) Retraction of single myelin sheaths during time in

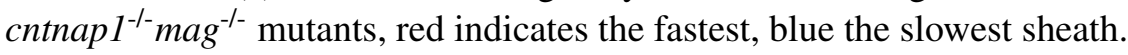
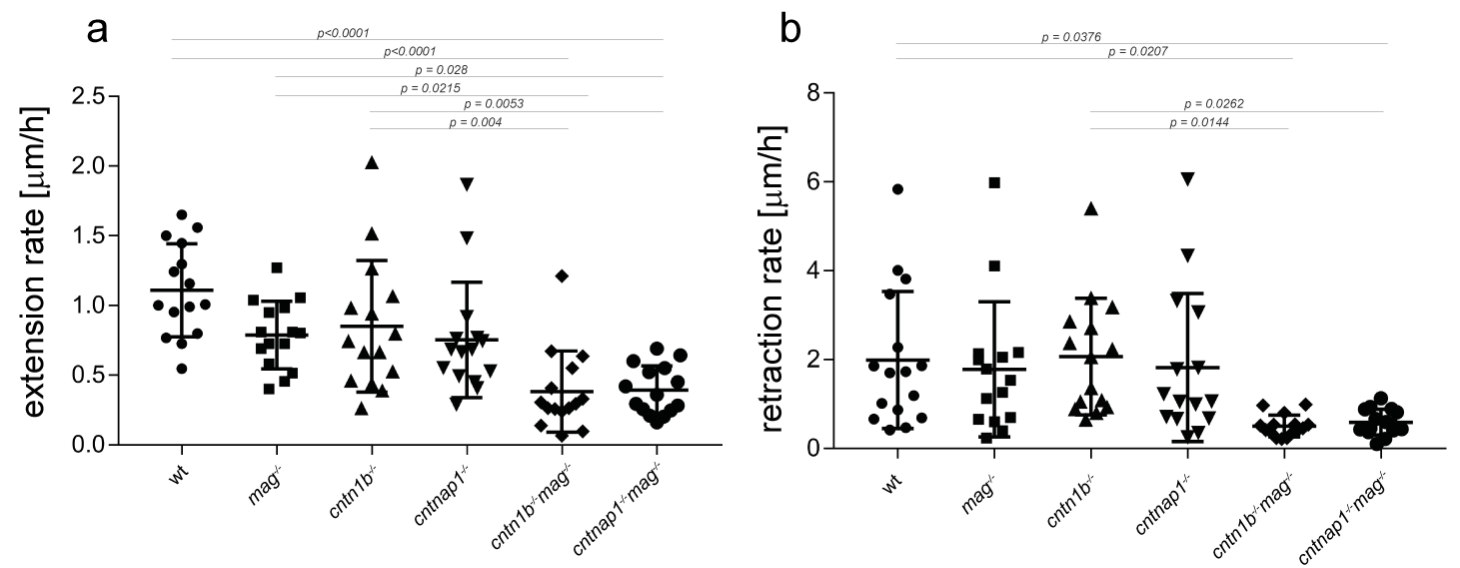

Figure 11: Quantification of extension and retraction rates (a) Average extension rates of single and double mutants (b) Average retraction rates of single and double mutants; in all quantifications we performed ANOVA, followed by pairwise t-test with p-value adjustment by Bonferroni method. 

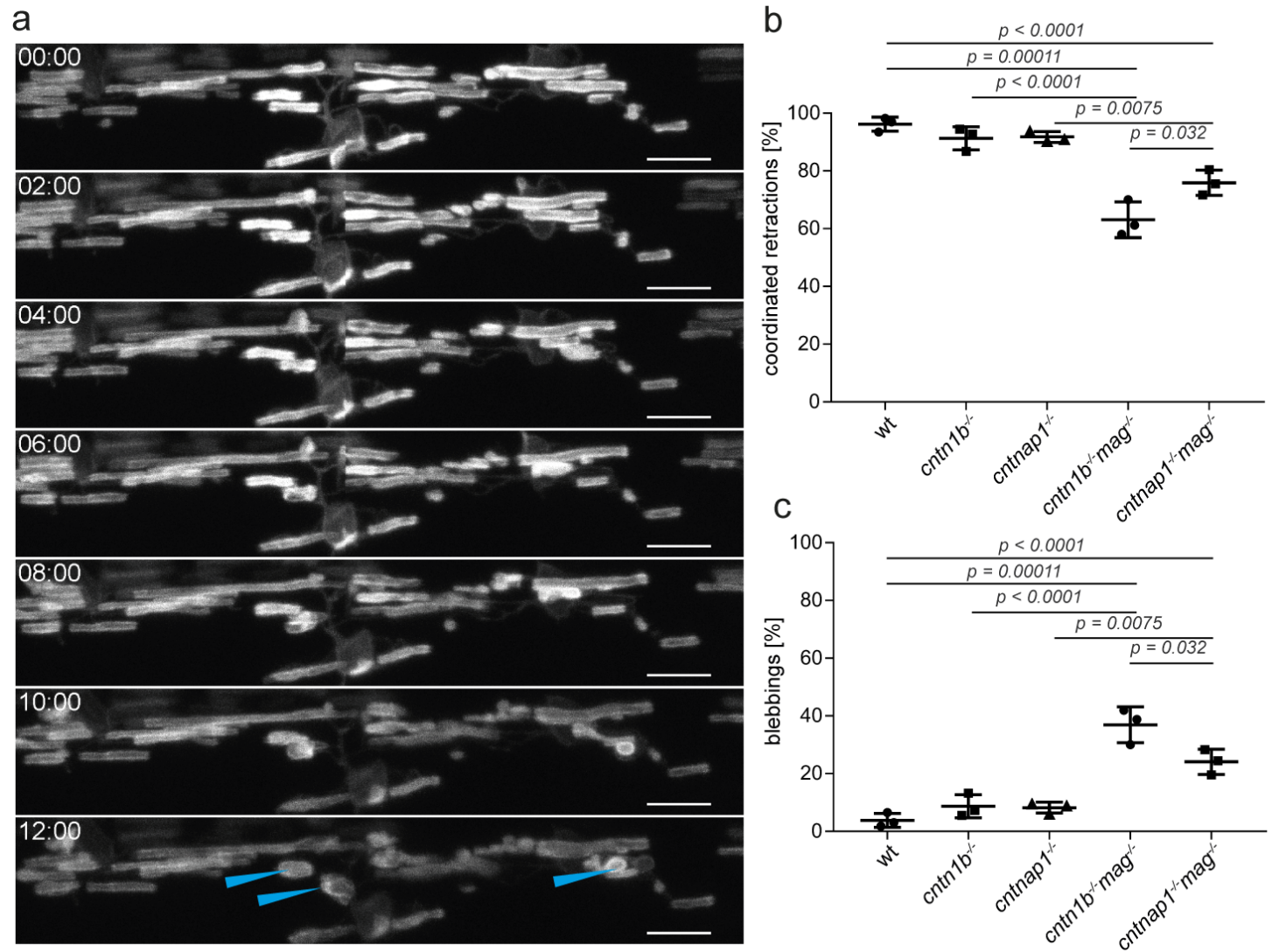

Figure 12: Blebbing of mutant myelin sheaths (a) Micrographs of a sheath disintegration event (blebbing) indicated by blue arrowheads, scalebar $=10 \mu \mathrm{m}$ (b) Quantification of coordinated retractions during $12 \mathrm{~h}$ (c) Quantification of blebbing events occured during $12 \mathrm{~h}$; in all quantifications we performed ANOVA, followed by pairwise t-test with p-value adjustment by Bonferroni method. 

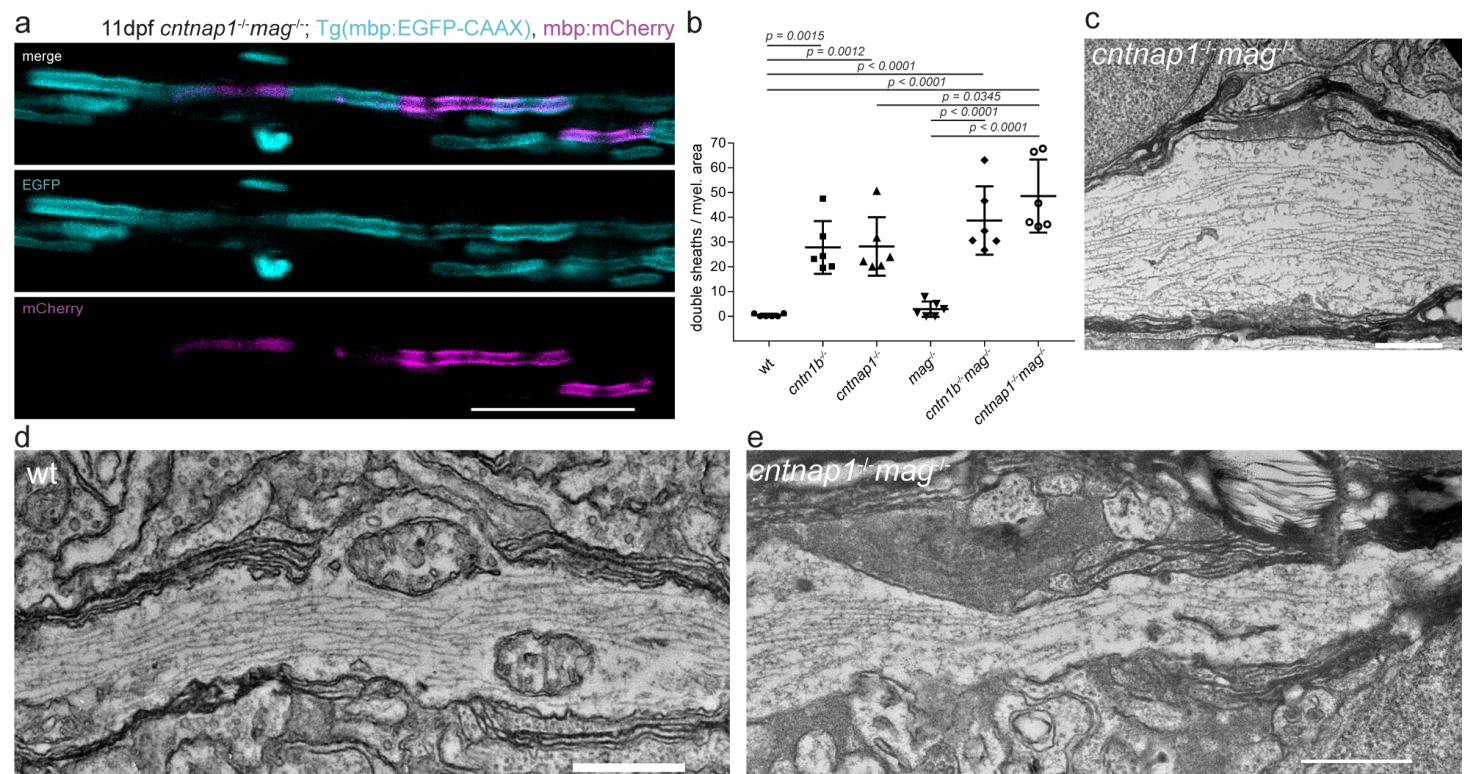

Figure 13: Doubled layered myelin in fish (a) Confocal micrographs of overlapping myelin sheaths, demonstrated by expression of mbp:mcherry in $\mathrm{Tg}$ (mbp:eGFP-CAAX fish), scale bar $=20 \mu \mathrm{m}$ (b) Quantification of double myelin across mutants, identified by $100 \%$ intensity step (c) TEM micrograph of an overgrown node in a longitudinal

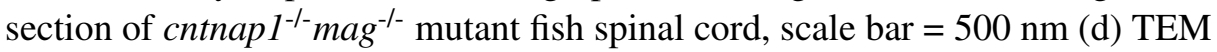
micrograph of a node of Ranvier in a longitudinal section of wildtype fish spinal cord, scale bar $=500 \mathrm{~nm}$ (e) TEM micrograph of distorted loop organization in a longitudinal section of cntnap $^{-I_{-}} \mathrm{mag}^{-{ }^{-}}$mutant fish spinal cord, scale bar $=500 \mathrm{~nm}$. 


\subsection{Mag together with the paranodal adhesion complex regulate targeting and wrapping of murine myelin sheaths}

\subsubsection{Conventional electron microscopy - mouse optic nerve cross sections}

Ultrastructural analysis by electron microscopy is well established in mouse optic nerve tissue. Since fixation of fish tissue exhibits several problems including fixation artifacts, we decided to run the ultrastructural analysis on mice.

\section{Reducing adhesion in developmental stages of $\mathrm{Cntn1}^{-/-} \mathrm{Mag}^{-/-}$mutants}

Heterozygous Cntn1 ${ }^{+/-}$single mutants (C57/B6N background) were purchased from Jackson laboratories. Cntn1 mutants develop gait impairments, ataxia and tremor increasing earlier than Cntnapl mutants leading to death at around P13. Cntn1 ${ }^{+-}$were cross bred with homozygous $\mathrm{Mag}^{-/-}$mice (C57/B6N background, kindly provided by Hauke Werner). Surprisingly, combined mutants together with Mag showed improvement of symptoms and could been kept until P16. We decided to set the latest observable timepoint at P12 for single and P15 for double mutants. Optic nerves were taken at P12 and P15 and conventionally fixed. Cross sections were analyzed for general myelin parameters. $\mathrm{CntnI}^{-{ }_{-}} \mathrm{Mag}^{-/-}$animals showed reduced numbers of myelinated axons per field of view at both timepoints (P12: 24.7 $\pm 4.8, \mathrm{P} 15: 52.8 \pm 8.7)$ compared to single mutants (Cntn1 ${ }^{-/} \mathrm{P} 12$ : 53.6 $\left.\pm 3.6, \mathrm{P} 15: \mathrm{NN}, \mathrm{Mag}^{-/-} \mathrm{P} 12: 63.7 \pm 10.0, \mathrm{P} 15: 83.8 \pm 12.9\right)$ and wildtype controls (P12: 78.6 \pm 8.1 , P15: 108.8 \pm 14.9 ) (see Fig.14a-b). The g-ratio in double mutants is slightly higher $(0.75 \pm 0.01)$ than controls $(0.71 \pm 0.02)$ and a fraction of small axons seems to be less myelinated than in wildtype controls (see Fig.14c-e).

Several myelin defects were observed in P12 and P15 mutant optic nerve cross sections. We quantified single myelinated axons, degenerated sheaths (whorls), split sheaths and outfoldings (tomaculi) (see Fig.15). At P12, in $\mathrm{CntnI}^{-{ }^{-}} \mathrm{Mag}^{-/-}$mutants, $52.8 \pm 8.4 \%$ of the axons per field of view were single myelinated. In contrast, $14.2 \pm 2.1 \%$ appeared as whorls, $20.1 \pm 5.4 \%$ as split sheaths and $12.9 \pm 4.7 \%$ as tomaculi. Single mutants and wildtype animals were almost similar among each other (wt: $97.0 \pm 0.5 \%$ single myelinated, $1.1 \pm 0.6 \%$ whorls, $0 \pm 0 \%$ 

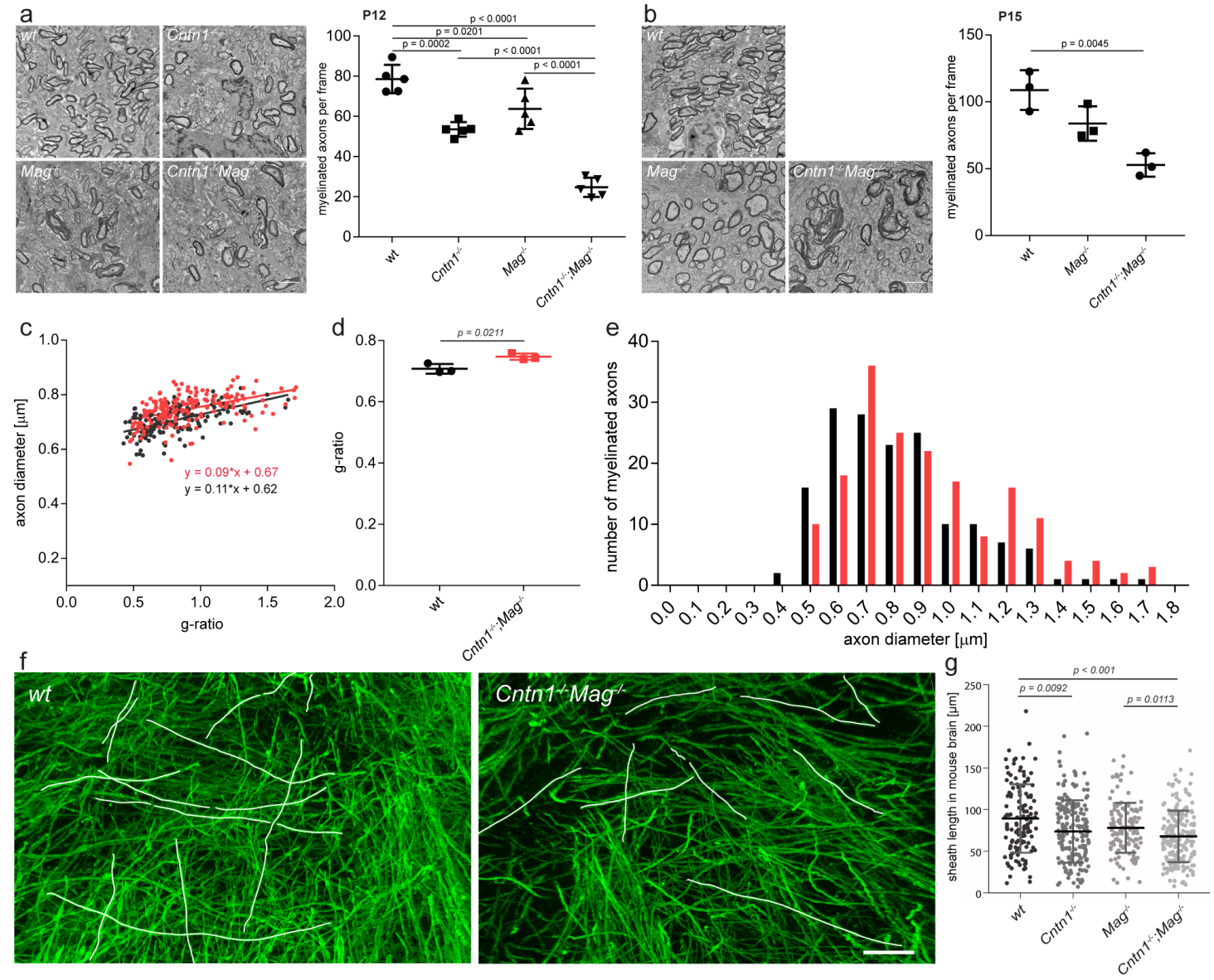

Figure 14: Properties of myelin in mouse mutants (a) TEM micrographs of optic nerve cross sections and quantification of myelinated axons at P12, statistical test: pairwise student's t-test, p-value adjustment by Bonferroni method (b) TEM micrographs of optic nerve cross sections and quantification of myelinated axons at P15, statistical test: pairwise student's t-test, $\mathrm{p}$-value adjustment by Bonferroni method (c) Quantification of optic nerve g-ratio to diameter plot of $\mathrm{CntnI}^{-/-} \mathrm{Mag}^{-/-}$mice and wildtype controls at P15 (d) Quantification of average g-ratio of both groups, statistical test: student's t-test (e) g-ratio histogram for both datasets (f) Representative confocal micrographs used to measure sheath lengths (g) Quantification of myelin sheath lengths in P12 single and double mutants, pairwise Wilcoxon rank sum test, p-value adjustment by Bonferroni method. 
split sheaths, $1.9 \pm 0.6 \%$ tomaculi; $\mathrm{Cntn}^{-{ }^{--}}: 91.5 \pm 2.2 \%$ single myelinated, $5.5 \pm 1.9 \%$ whorls, $0 \pm 0 \%$ split sheaths, $3.0 \pm 0.5 \%$ tomaculi; $\mathrm{Mag}^{-/-}: 92.5 \pm 0.1 \%$ single myelinated, $2.7 \pm 1.0 \%$ whorls, $2.5 \pm 0.9 \%$ split sheaths, $2.3 \pm 0.3 \%$ tomaculi). At P15, CntnI ${ }^{-/-} \mathrm{Mag}^{-{ }^{--}}$mutants showed $61.7 \pm 7.3 \%$ single myelinated axons per field of view. $10.4 \pm 4.4 \%$ appeared as whorls, $22.0 \pm 2.2 \%$ as split sheaths and $6.0 \pm 2.1 \%$ as tomaculi. Single mutants and wildtype animals were almost similar among each other (wt: $97.7 \pm 0.25 \%$ single myelinated, $1.1 \pm 0.6 \%$ whorls, $0 \pm 0 \%$ split sheaths, $1.2 \pm 0.4 \%$ tomaculi; $\mathrm{Mag}^{-/-}: 93.3 \pm 1.8 \%$ single myelinated, $1.7 \pm 0.5 \%$ whorls, $2.3 \pm 0.5 \%$ split sheaths, $2.7 \pm 1.1 \%$ tomaculi). Double mutants in general show more aberrant myelin structures than single mutants or wildtype controls.

Since co-depletion of paranodal junction complex and Mag causes double myelin sheaths in mice and fish, we tried to assess if lateral growth is impaired too. Therefore we used immunohistochemistry of thick brain sections $(300 \mathrm{~nm})$ with long incubation time. Brains were extracted from P12 wildtype animals, $\mathrm{Mag}^{- \text {- }^{-}}, \mathrm{CntnI}^{-\mathrm{I}_{-}}$single and $\mathrm{CntnI}^{-\mathrm{I}_{-}} \mathrm{Mag}^{-\mathrm{I}_{-}}$double mutants (2 per group, kindly provided by Dr. Minou Djannatian), PFA-fixed and sectioned in PBS using a vibratome. Stainings for Mbp were applied and large areas of the thalamus were scanned. Using 3D-analysis software (bitplane IMARIS) we measured the lengths of all myelin sheaths with a distinct start and end point within the borders of the scanned volume. P12 thalamic myelin sheath lengths were reduced in $\mathrm{CntnI}^{-{ }_{-}^{-}} \mathrm{Mag}^{- \text {-- }^{-}}$mutants $(67.7 \pm 30.9 \mu \mathrm{m})$ and $\mathrm{CntnI}^{-{ }_{-}}$animals $(73.6 \pm 37.3 \mu \mathrm{m})$ compared to control mice $(89.2 \pm 41.1 \mu \mathrm{m})$ and the $\mathrm{Mag}^{-/-}$single mutants $(78.0 \pm 29.9 \mu \mathrm{m})($ see Fig.14).

\section{Reducing adhesion in later stages of Cntnap1-- $\mathbf{M a g}^{-/-}$and Cntnap1/-- Cntnap2 ${ }^{-/-}$Mag $^{-/-}$mutants}

$\mathrm{CntnI}^{-/-}$mice die at around P13/14 postnatal. In wildtype mice, myelination of the optic nerve is not finished at this point, therefore the data acquired in this model is refelecting developmental stages. Cntnap $1^{-/-}$animals can live at least until around P23. Since optic nerve myelination in unaffected animals is finished until P21, we could use the Cntnap1 ${ }^{-/-}$model to assess the consequences of paranodal junction disruption at later stages of myelin development. Addition- 

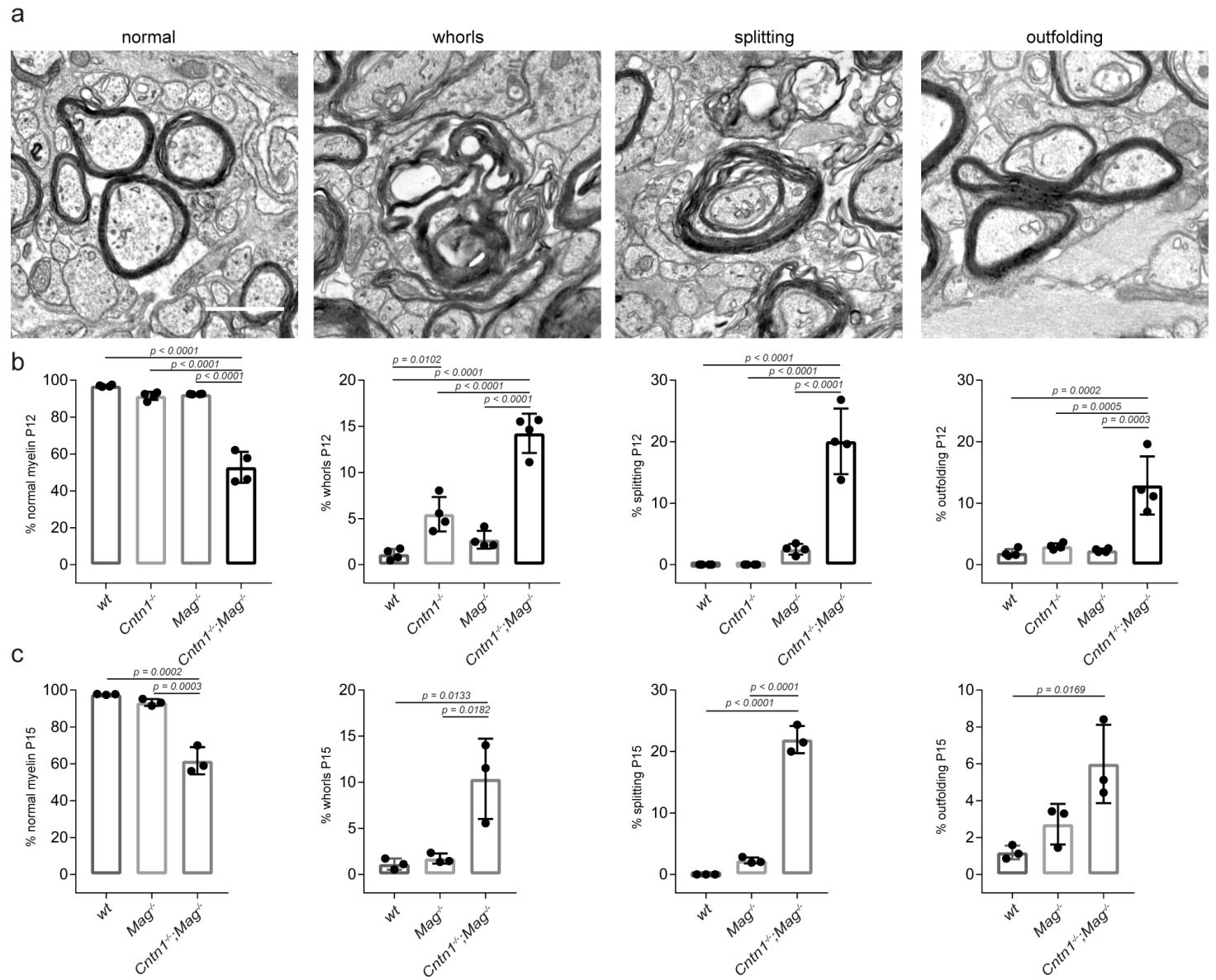

Figure 15: Myelin defects present in $\boldsymbol{C n t n I}^{-/-} \mathbf{M a g}^{-/-}$double mutants (a) Representative TEM micrographs of myelinated axons, degenerated myelin (whorls), split myelin and outfoldings (tomaculi) at P15 (b) Quantification of myelin defects at P12 in \% of all myelinated axons observed in an individual (c) Quantification of myelin defects at P15 in \% of all myelinated axons observed in an individual; in all quantifications we performed ANOVA, followed by pairwise t-test with p-value adjustment by Bonferroni method. 
ally, Cntnap $2^{+/-}$mutants were used to generate a Cntnap $1^{-/-}$Cntnap $^{-{ }^{-/}}$Mag $^{-/-}$triple knock out animal with neuron-glia adhesion reduced to a minimum. Heterozygous $\mathrm{Cntnap1}^{+/-} \mathrm{Cntnap2}^{+/-}$ double mutant animals (NMRI backgroungd, kindly provided by Elior Peles) were cross bred with homozygous Mag-null mice (C57/B6N background, kindly provided by Hauke Werner). Mag single mutant mice are indistinguishable from wildtype mice and exhibit only subtle impairments increasing with age. Cntnap1 and Cntnap2 mutants develop progressive gait impairments, ataxia and tremor starting at about P15 increasing with development and leading to death at around P23.[175] Combined mutants developed slightly worse symptoms at the same onset. Every animal was weighted before been sacrificed (see Fig.16). At P12/13, no weight difference can be observed between wildtype, single or combined mutants (all about 8 to 9 g). At P21/22, physical impairments are reflected in the weight. Mice lacking Cntnapl are significantly lighter (7.6 $\pm 2.5 \mathrm{~g})$ than wildtype mice(11.5 $\pm 2.3 \mathrm{~g})$. Depletion of Cntnapl together with Mag decreases the weights compared to single mutants, whereas additional knock out of Cntnap2 has no effect (Cntnap1 $1^{-/-} \mathrm{Mag}^{-/-}: 5.4 \pm 1 \mathrm{~g}$, Cntnap1 ${ }^{-/-}$Cntnap2 $\left.^{-{ }^{--}} \mathrm{Mag}^{-/-}: 5.1 \pm 0.9 \mathrm{~g}\right)$. According to German regulations and human endpoint decisions we decided to set the latest observable timepoint at P22.

Optic nerve samples of Cntnap1, Cntnap2, Mag mutants and its combinations were taken at $\mathrm{P} 12 / 13$ and P21/22. TEM micrographs of cross sections revealed reduced numbers of myelinated axons at P12/P13 in all mutants lacking Mag (see Fig.19 and Fig.20). At P21/22, hypomyelina-

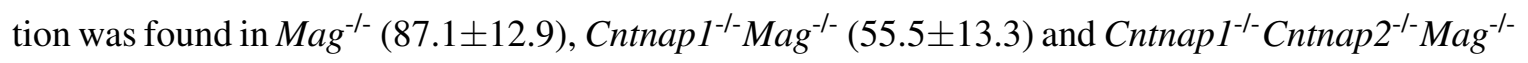
(59.05 \pm 5.4$)$ mutants compared to wildtype (160.98 \pm 24.8$)$. We additionally counted myelinated and unmyelinated axons in half of the micrographs of P21/22 animals (see Fig.17a). In

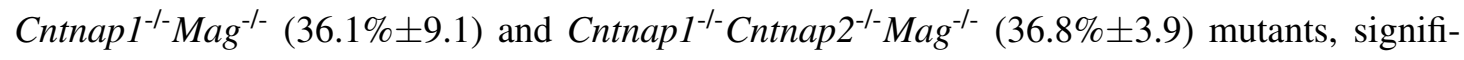
cantly less axons were myelinated compared to wildtype $(65.3 \% \pm 2.7)$ or their single mutant counterparts (Cntnap ${ }^{-/-}: 67.9 \pm 7.5$, Cntnap2 $2^{-/-}: 63.7 \% \pm 11.5$ Mag $^{--_{-1}}: 53.9 \% \pm 8.2$ ). The thickness of the myelin sheath can be described as the g-ratio (axon diameter/myelin diameter). We measured the area occupied by axon and myelin sheaths only of myelin sheaths that did not 
a

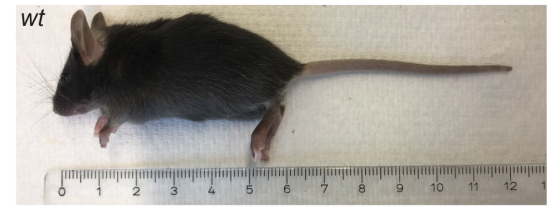

b
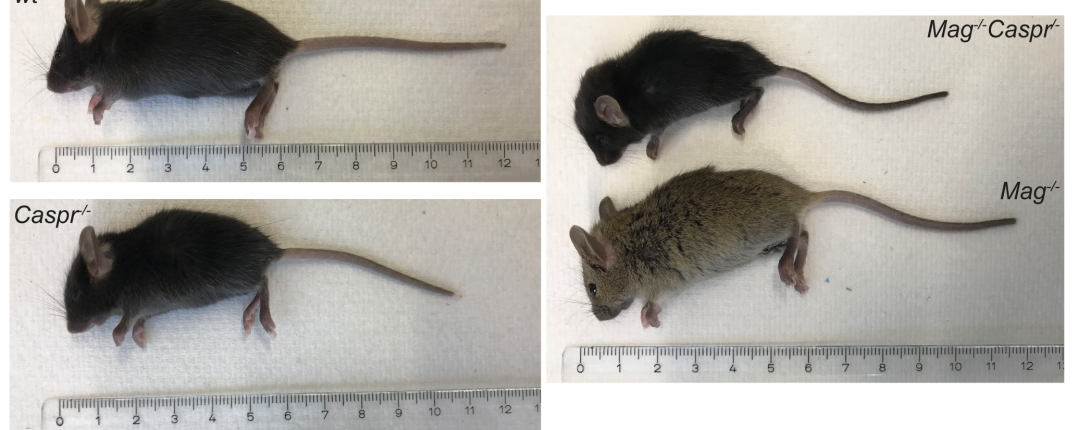

C
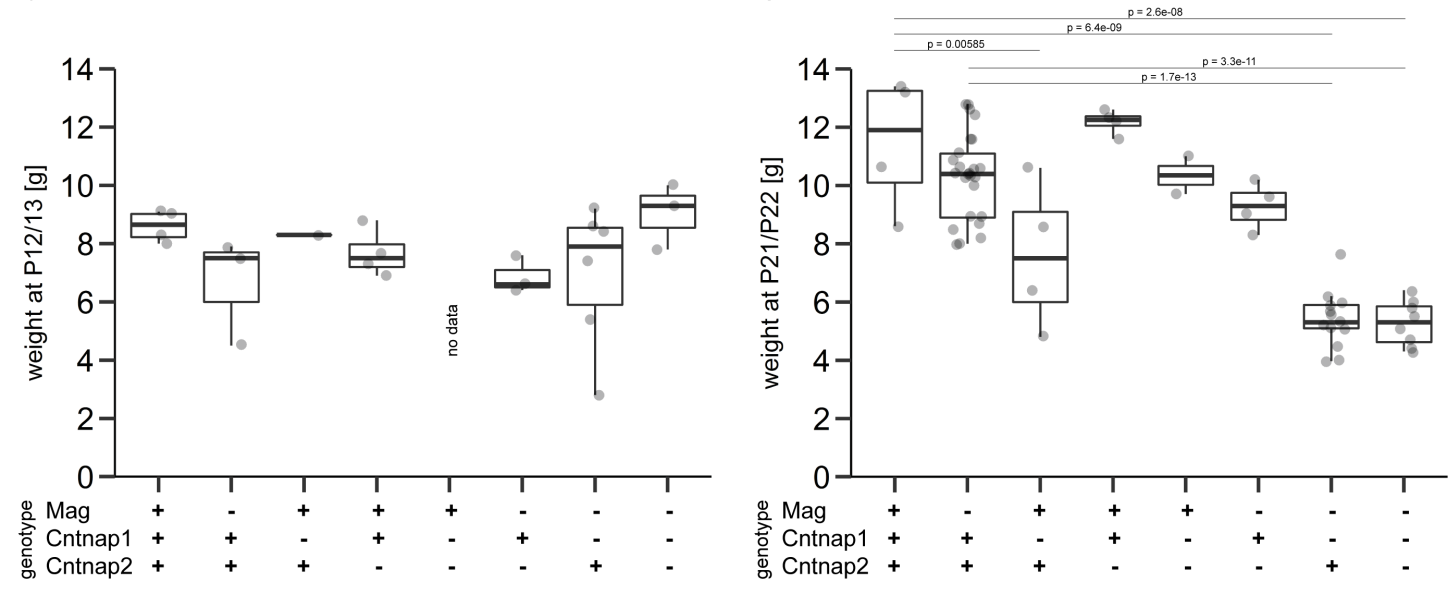

Figure 16: Weight of mutant mice (a) Images of representative mouse mutants and wildtype mouse at P21 (b) Quantification of weight at P12/13 (c) Quantification of weight at $\mathrm{P} 21 / 22$; in all quantifications we performed ANOVA, followed by pairwise t-test with p-value adjustment by Bonferroni method. 
exhibit abnormalities like degeneration or splittings. From the area we calculated the diameter using the formula for an ideal circle $(r=\sqrt{(} A / \pi))$. The g-ratio was then calculated as: $g=r_{\text {axon }} / r_{\text {myelin. }}$. The g-ratio was slightly lowered in double and triple mutants, indicating a trend to slightly thicker myelin sheaths (not significant, see Fig.17a). The relation of g-ratio to caliber showed that fewer axons in the diameter range of 0.4 to $0.5 \mu \mathrm{m}$ were myelinated in Cntnap ${ }^{-/-}$Cntnap2 $^{-/-}$Mag $^{-/-}$mice than in wildtype controls (see Fig.17b).

Similar to fish, the main feature observed in mutant optic nerve cross sections were split myelin sheaths. We quantified them in 10 micrographs of $219 \mu \mathrm{m}^{2}$ per animal. Myelinated cell bodies or dendrites could not be found in ultrastructural analysis nor in immunohistochemical staining for neuronal markers (Mbp, neurofilament (NF) together with neuron-specific nuclear protein $(\mathrm{NeuN})$ for cell bodies or microtubuli associated protein 2 (Map2) for dendrites, data not shown). In wildtype sections, split myelin appeared very rarely during development (P12/13) but was absent after myelination is completed (P21/22). At P12, split myelin was more

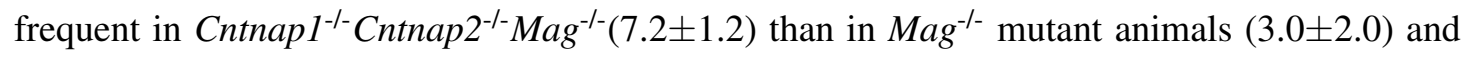
wildtype controls $(0.56 \pm 0.56)$. P21 mutant animals exhibited generally more split myelin than

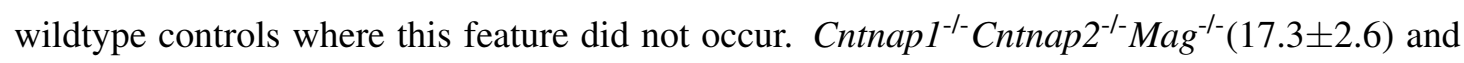

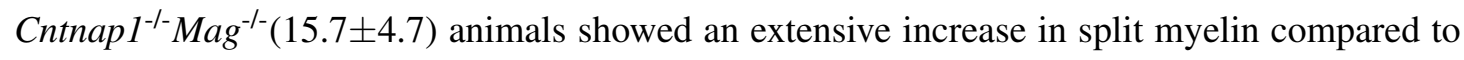
wildtype controls $(0.08 \pm 0.07)$ and all single mutants. The depletion of Cntnap2 had no additional effect when Cntnapl and Mag were already missing.

As later confirmed by longitudinal sectioning and 3D reconstruction, split myelin is often caused by double layers of myelin.

\subsubsection{Electron microscopy of high pressure frozen optic nerve longitudinal sections - Loss of the nodes of Ranvier}

Conventional fixation methods introduce many artifacts due to tissue shrinking or stretching. Fixation by high-pressure freezing offers an alternative that conserves the cellular ultrastruc-

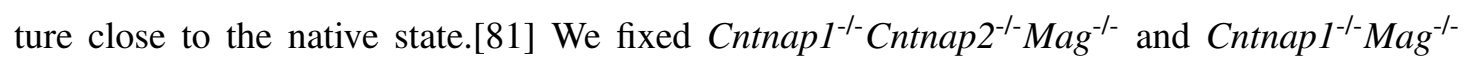



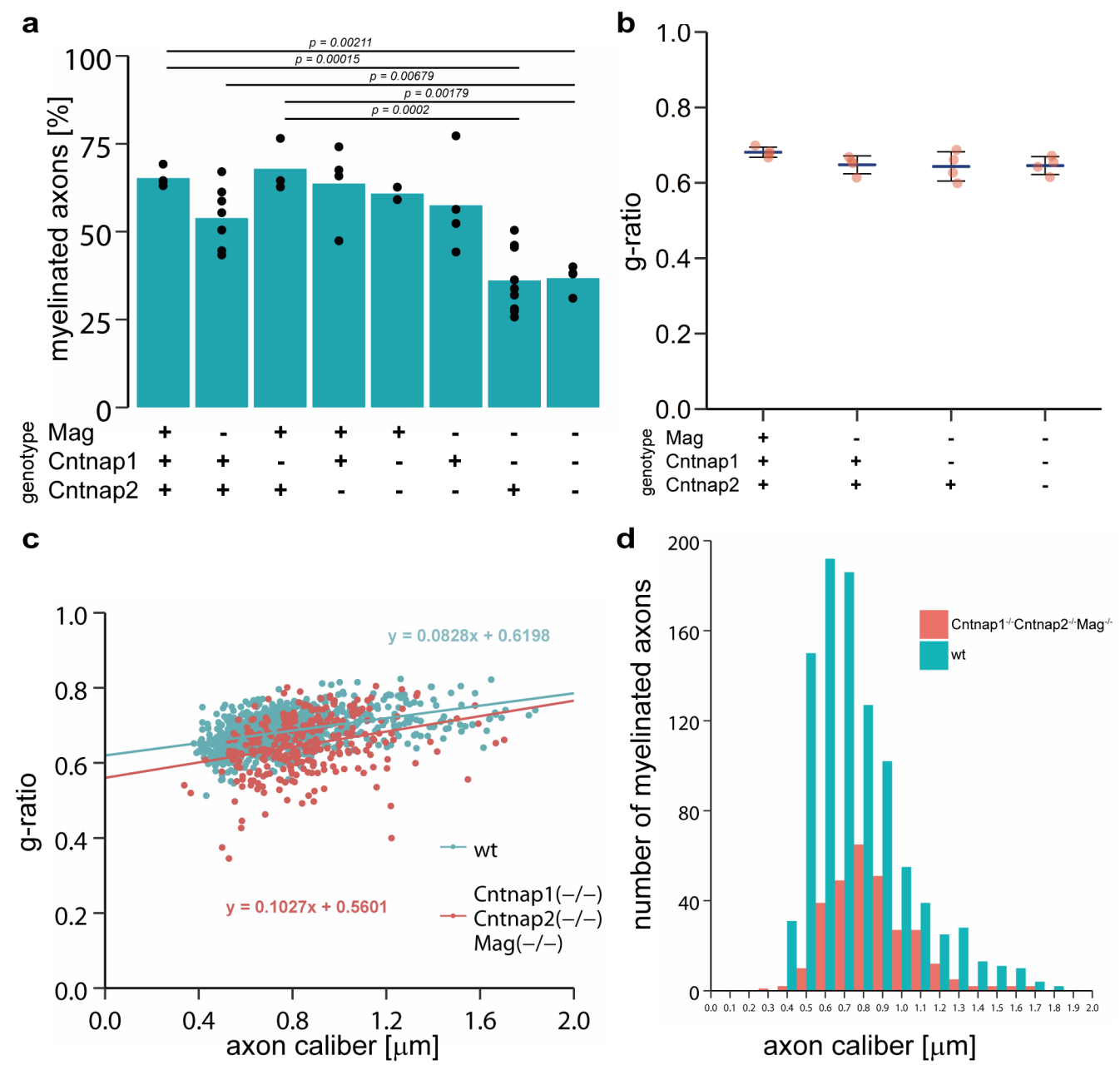

Figure 17: Analysis of myelination and g-ratios in mice (a) Relative quantification of myelinated and unmyelinated axons (b) Quantification of average g-ratio of mutant mice at $\mathrm{P} 21 / 22$ (c) g-ratio to caliber distribution for wildtype and

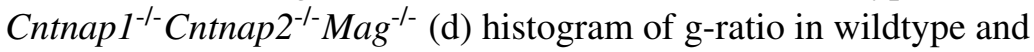
Cntnap $^{-/-}$Cntnap $^{-/-}$Mag $^{-/-}$animals, analysis of 5 frames $\left(219 \mu \mathrm{m}^{2}\right)$ per animal, $\mathrm{n}=4$; in all quantifications we performed ANOVA, followed by pairwise $\mathrm{t}$-test with p-value adjustment by Bonferroni method. 

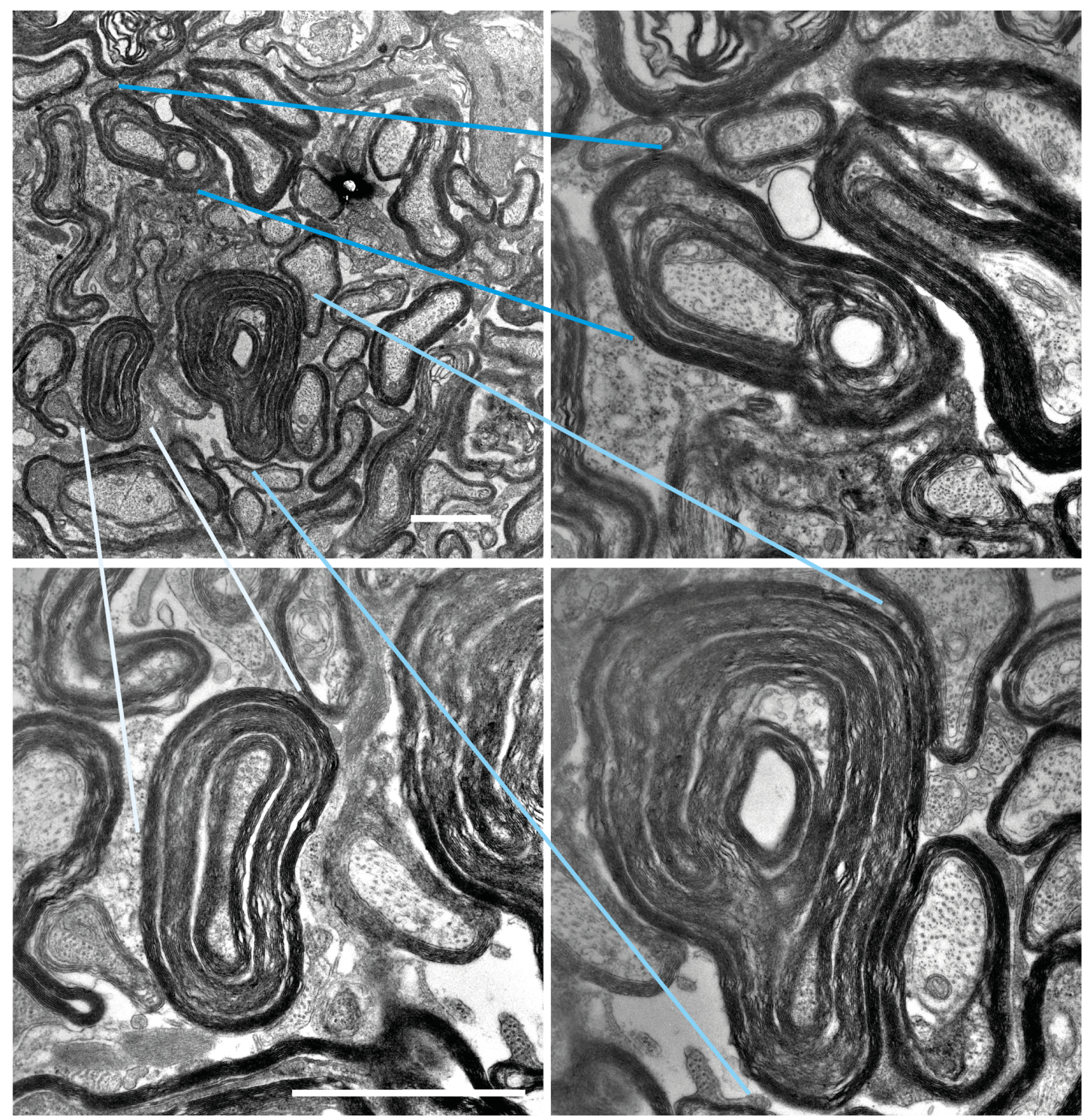

Figure 18: Representative electron micrograph and zoomed tiles P21

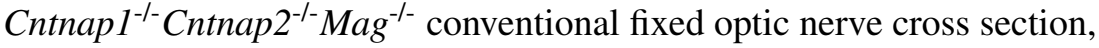
demonstrating multiply myelinated axons and outfoldings, scalebar $=2000 \mu \mathrm{m}$. 


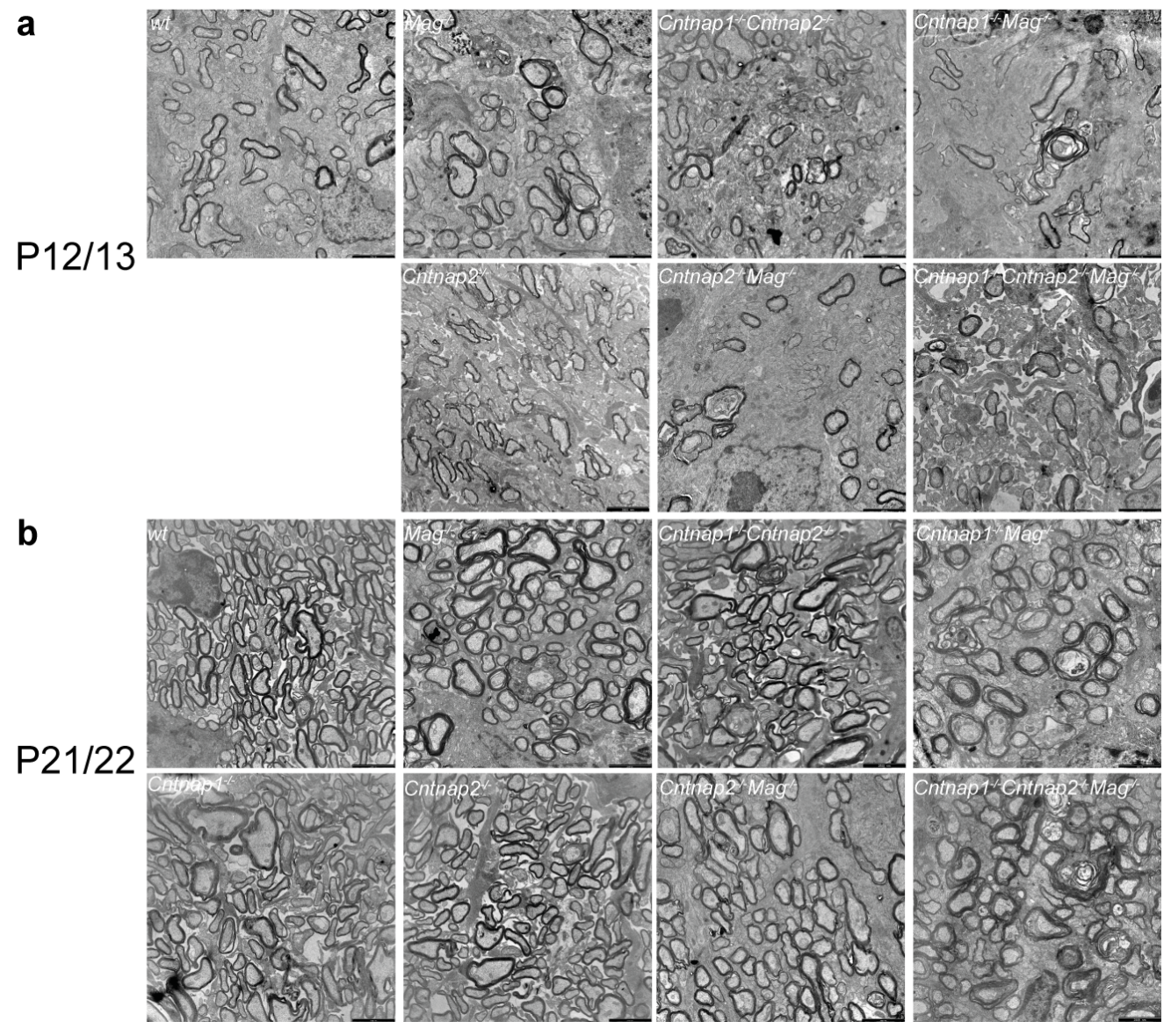

Figure 19: Representative electron micrographs of (a) P12/13 optic nerve cross sections,

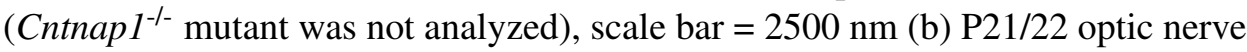
cross sections, scale bar $=2500 \mathrm{~nm}$. 

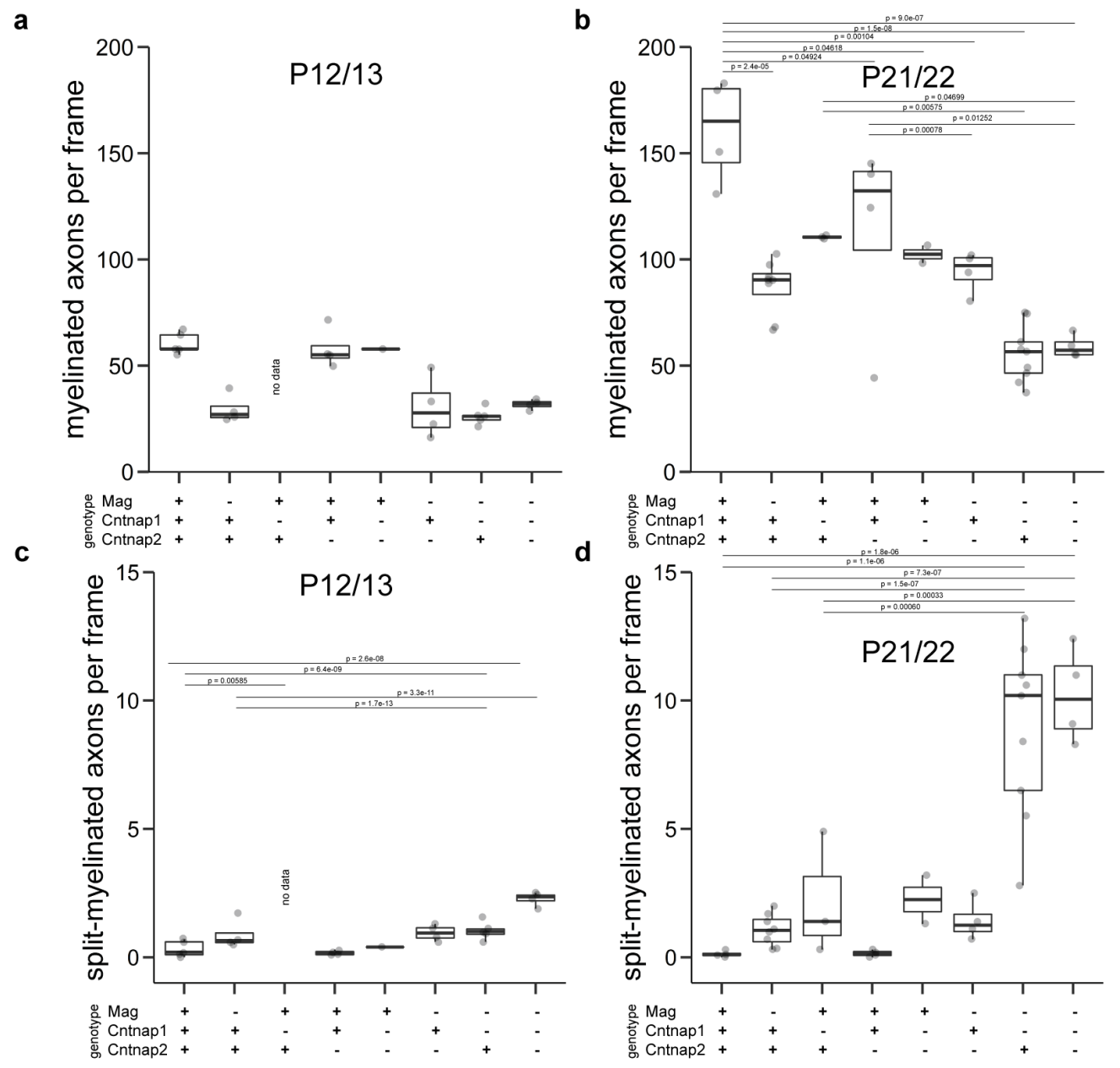

Figure 20: Quantification of single and split myelinated axons in mice (a) Quantification of myelinated axons at P12/13 (b) Quantification of myelinated axons at P21/22 (c) Quantification of P12/13 split-myelinated axons P12/13 (d) Quantification of myelinated axons at $\mathrm{P} 21 / 22$, analysis of 10 frames $\left(219 \mu \mathrm{m}^{2}\right)$ per animal; in all quantifications we performed ANOVA, followed by pairwise t-test with p-value adjustment by Bonferroni method. 
mouse optic nerves with high pressure freezing. The optic nerves were cut longitudinal, so looking at the axons from a different perspective and tracking them for some tens of microm-

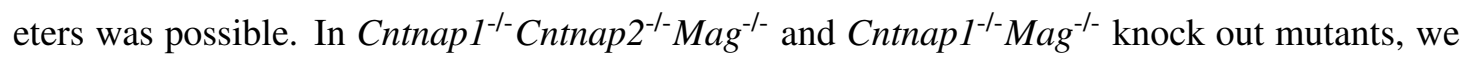
observed extensive double myelination reflected by paranodal loop-like structures on top of myelin sheaths or in contact with the axon but burried under compact myelin. Former nodes of Ranvier, that grew into each other was another striking observation. Single mutants lacking only Cntnap1, Cntnap2 or Mag and the combined mutants of Cntnap2 and Mag showed aberrant myelin structures: detached, disordered or enlarged paranodal loops and degenerated myelin (onion bulbs). It is noteworthy, that regulary ordered nodes were found in all single mutants

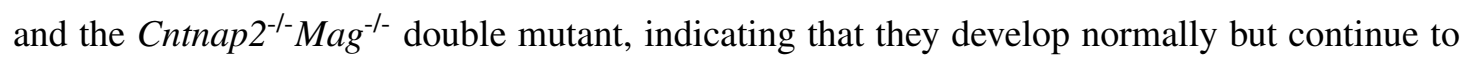
grow. To quantify aberrant myelination, longitudinal high-pressure-frozen sections of the optic nerve were analyzed by TEM. One grid hexagon $\left(26.000 \mu \mathrm{m}^{2}\right)$ served as the standardized area of view. According to our observations, we defined 4 categories: [I] intact node of Ranvier, [II] solitary sheath end, [III] paranodal loop-like structure on top of an existing myelin sheath and [IV] paranodal loop-like structure under an existing myelin sheath and counted numbers per field of view (see Fig.23). Numbers of solitary sheath ends are sligthly higher in mutant

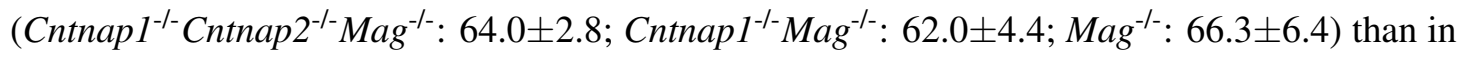
wildtype animals $(47.3 \pm 5.5)$.

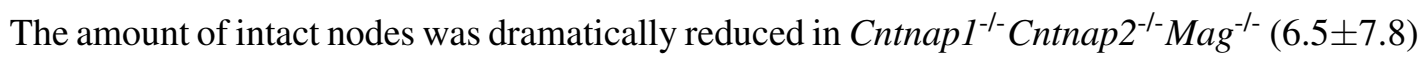
and Cntnapl ${ }^{-/} \mathrm{Mag}^{-/-}(6.0 \pm 5.6)$ mice, compared to Mag mutants (43.3 \pm 9.0$)$ and wildtype controls (64.3 \pm 9.0$)$. The aberrant myelin structures resembling former paranodal loops [III] and [IV] were absent in wildtype ([III]: $0 \pm 0$; [IV]: $0 \pm 0$ ), extremly rare in Mag single mutants ([III]:

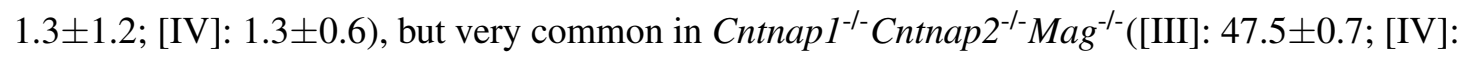
32.5 \pm 23.3 ) and Cntnapl $^{-/-}$Mag $^{-/-}$mice ([III]: 58 \pm 7.8 ; [IV]: 19.0 \pm 8.2 ).

Extended overgrowth of nodes of Ranvier raises the question of the fate of the sodium channels, usually localized nodally. To answer that by localizing and quantifying the signal transmitting sodium channels, we used immunohistochemistry. Longitudinal cryo-sections of PFA- 

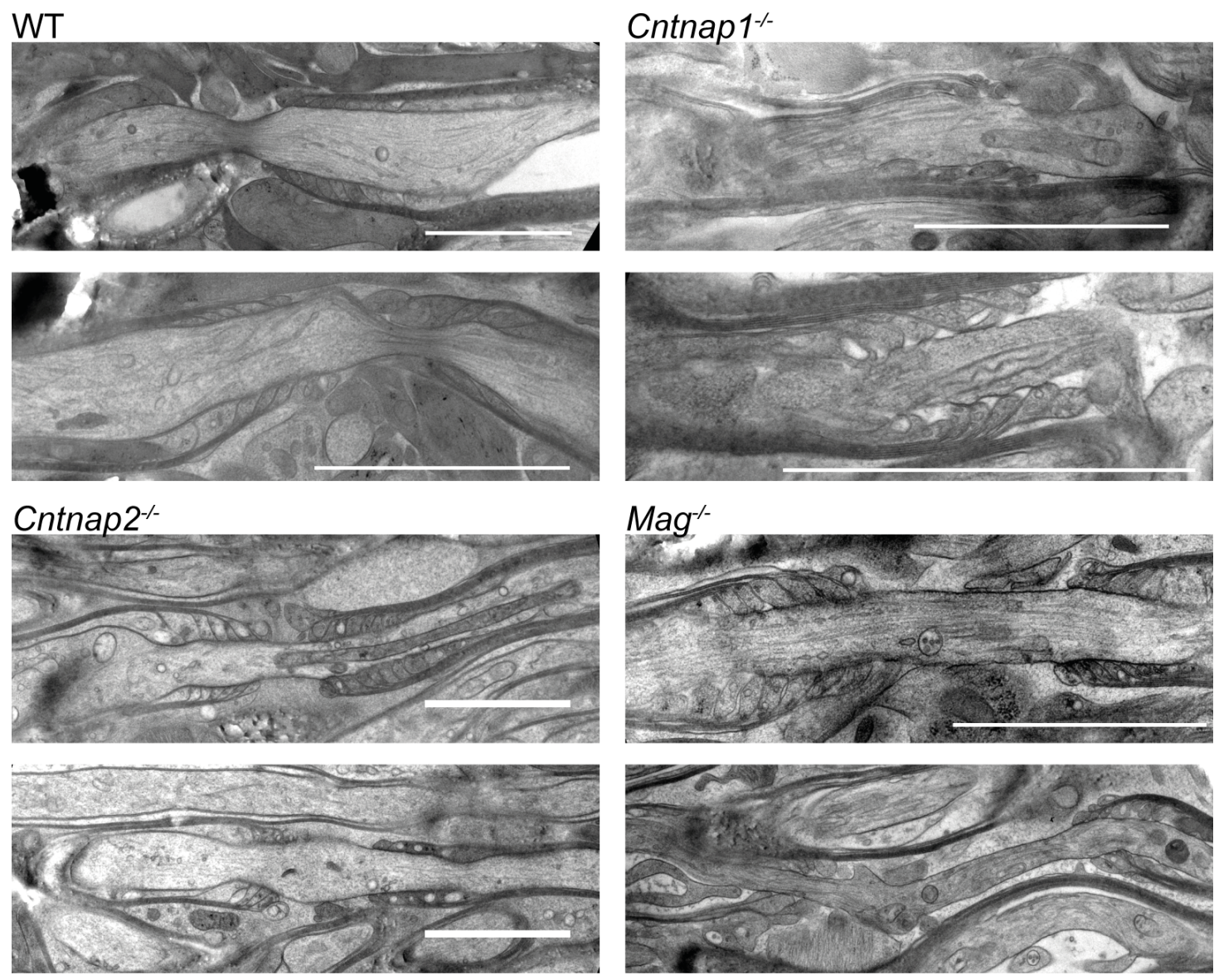

Figure 21: Electron micrographs of P21 single mutant and wildtype mice optic nerves, high pressure freezing fixation, showing nodes of Ranvier with paranodal loops, scalebar $=2 \mu \mathrm{m}$.

fixed optic nerves were stained for sodium channel $\mathrm{Na}^{\mathrm{v}} 1.6$ and neurofilament. $\mathrm{Na}^{\mathrm{v}} 1.6$ chan-

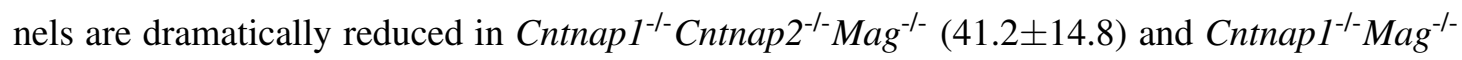
(62.4 \pm 51.1$)$ animals compared to $\mathrm{Mag}^{-/-}(175.4 \pm 53.8)$ single mutants and wildtype controls (180.5 \pm 44.0$)$. Properly formed nodes containing $\mathrm{Na}^{\mathrm{v}} 1.6$ were nearly absent when Cntnapl and Mag were co-depleted (see Fig.24). 

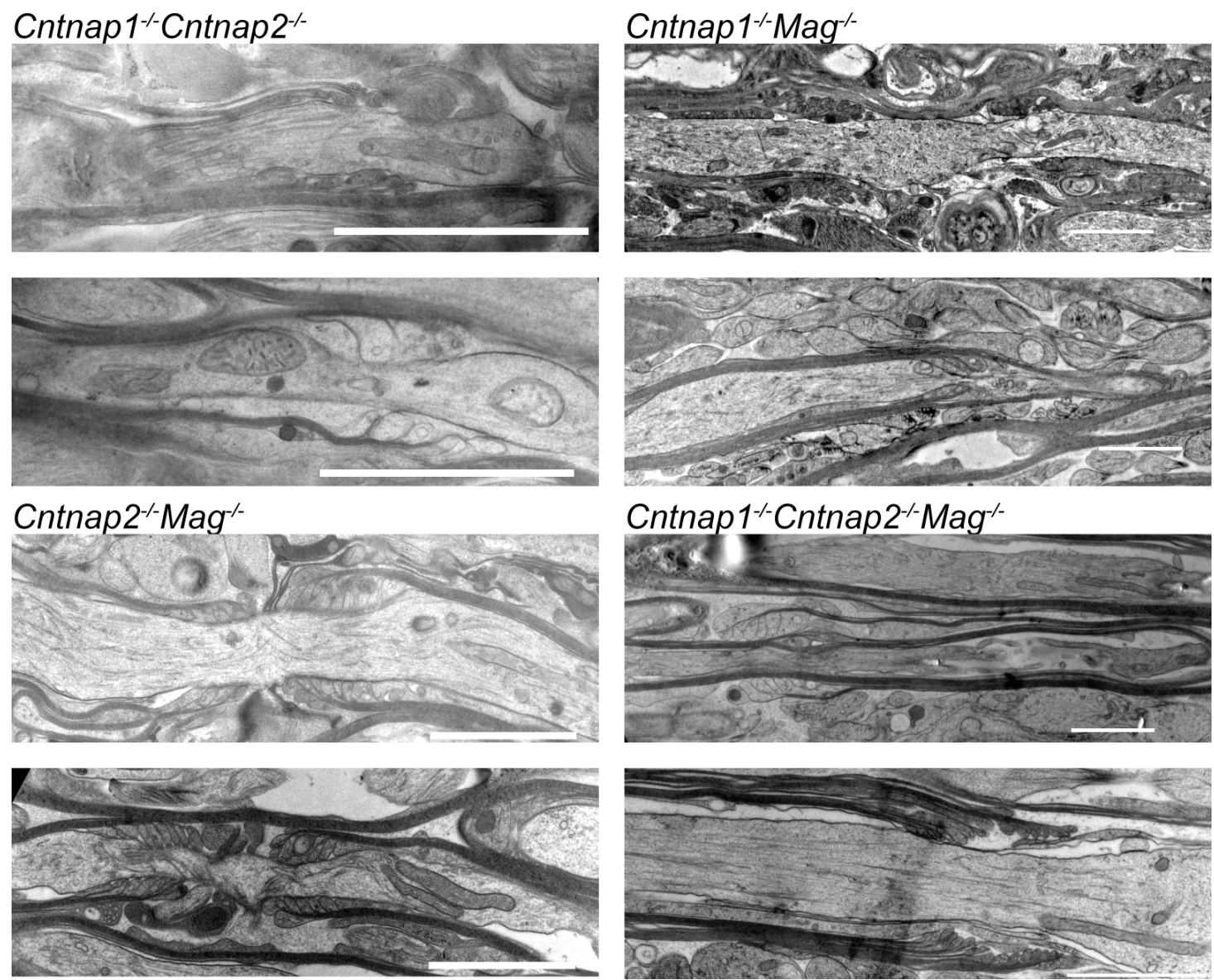

Figure 22: Electron micrographs of P21 double and triple mutant mice optic nerves, high pressure freezing fixation, showing nodes of Ranvier with paranodal loops and paranodal loop like structures, scalebar $=2 \mu \mathrm{m}$. 

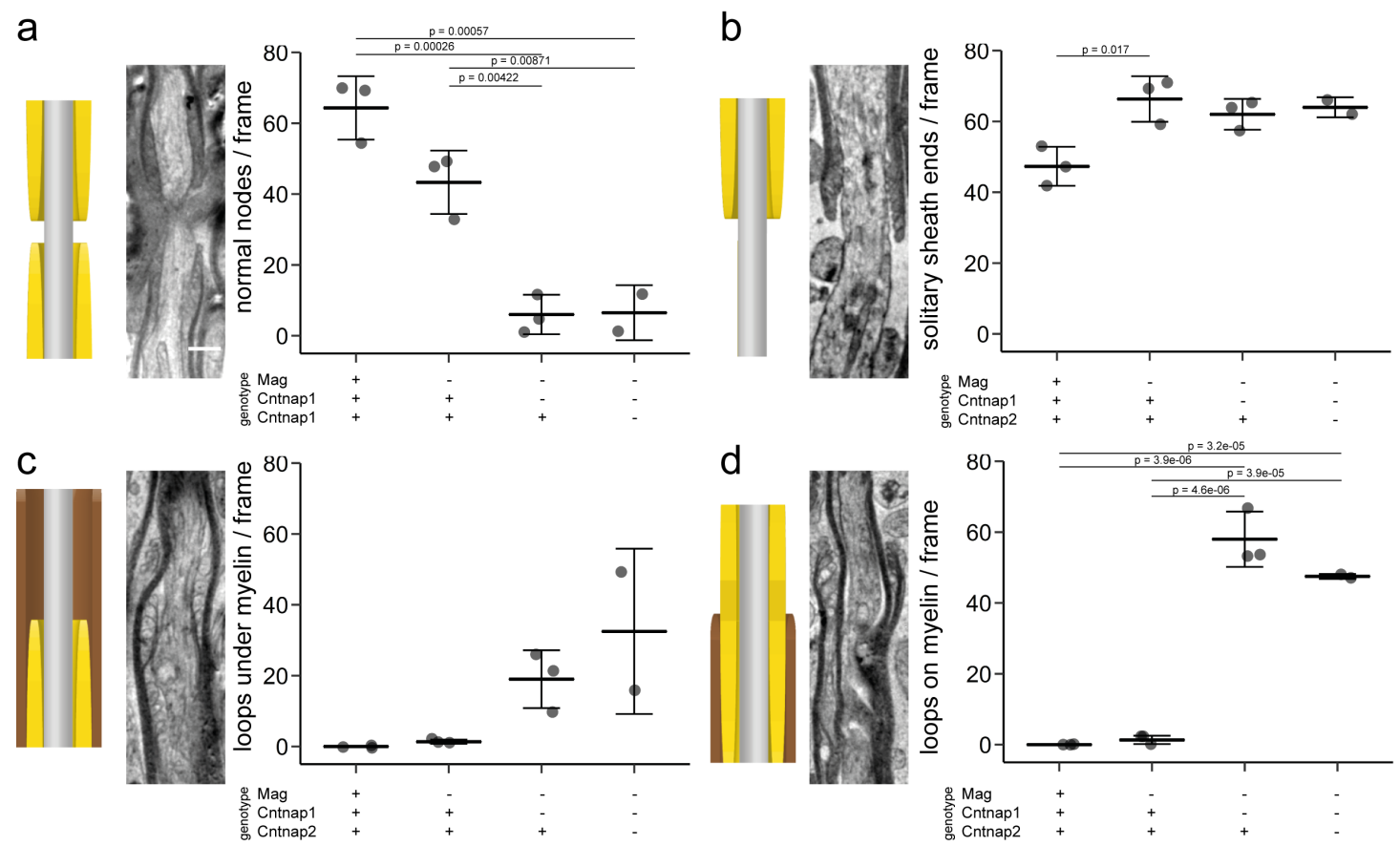

Figure 23: Model, representative image and quantification of (a) [I] normal nodes (b) [II] solitary sheath ends (c) [III] loops under myelin (d) [IV] loops on myelin, scalebar = $1000 \mathrm{~nm}$; pairwise t-test, p-value adjustment by Bonferroni method.

a

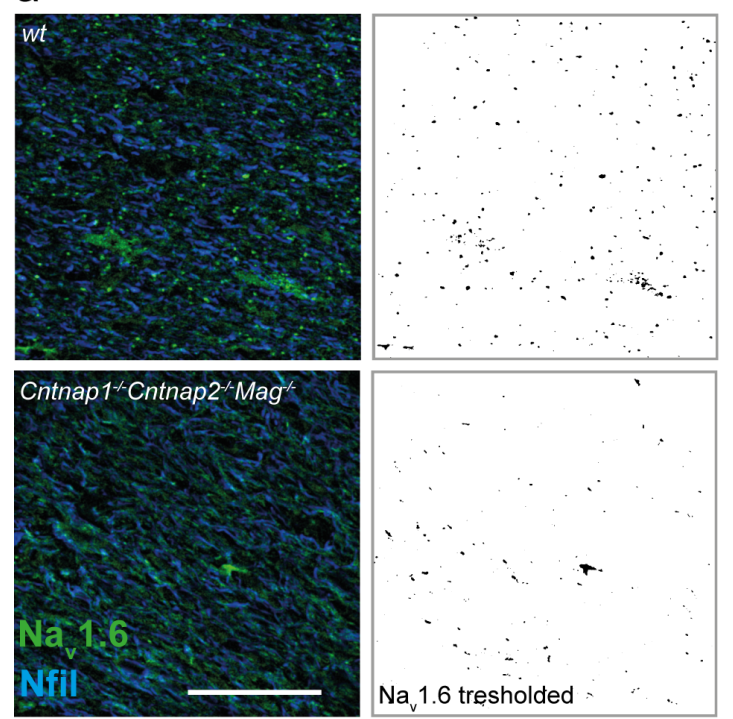

b

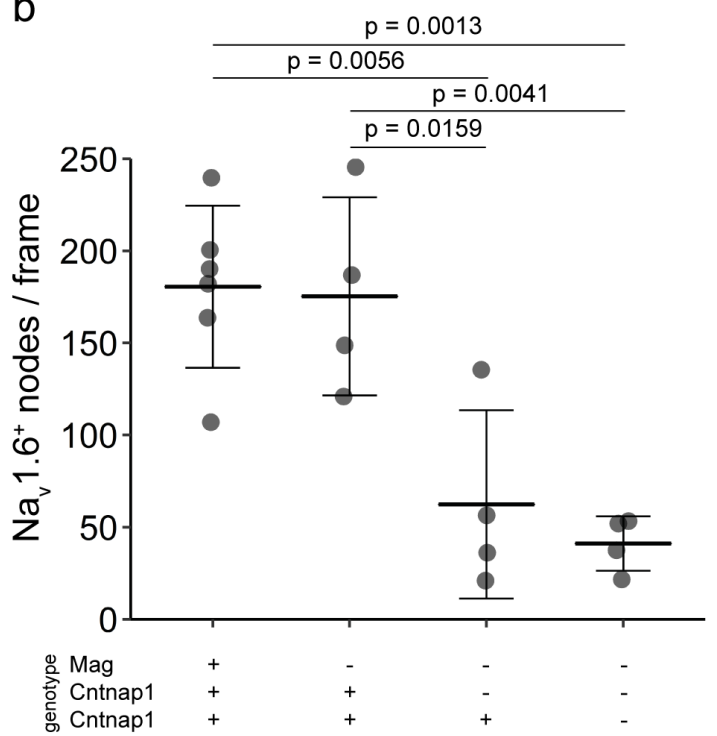

Figure 24: $\mathbf{N a}_{\mathbf{v}} \mathbf{1 . 6}$ staining in wildtype and mutant animals (a) Representative confocal micrographs showing $\mathrm{Na}_{\mathrm{v}} 1.6$ positive signals in mutant and wildtype optic nerve longitudinal sections, scale bar $=50 \mu \mathrm{m}$ (b) Quantification of nodes of Ranvier; ANOVA followed by pairwise t-test, p-value adjustment by Bonferroni method. 


\subsubsection{D reconstruction of optic nerve multiply myelinated axons}

The amount of information 2D images can provide is limited. 3D reconstruction of biological structures allows a multi-perspective view and holistic understanding of the observations. Within this study, we used the advantages of two complementary methods: ATUM and FIB-SEM. ATUM is a 3D method offering a large field of view (especially in z-direction) at relatively low resolution. With FIB-SEM the resolution is within the nanometer scale, but the field of view and the volume is limited. To assess the biology of split myelin sheaths, we performed ATUM on conventionally fixed $\mathrm{CntnI}^{-/-} \mathrm{Mag}^{-/-} \mathrm{P} 15$ optic nerves. A volume of $25 \mu \mathrm{m} \times 25 \mu \mathrm{m}$ x $55 \mu \mathrm{m}$ was analyzed and revealed, that many axons that present split myelin, can either be double myelinated or categorized to the "inside-out-wrapping" type. For some axons carrying split myelin it was not possible to distinguish (ambiguous). Within this volume, we labeled axons presenting: single myelin sheaths, double myelin sheaths, myelin of the "inside-out wrapping" type and ambiguous sheaths (see Fig.25). A volume of $20 \mu \mathrm{m} \times 15 \mu \mathrm{m} \times 17.5 \mu \mathrm{m}$ was analyzed by FIB-SEM to get detailed 3D-structure information about the "inside-out-wrapping" type. Two inner tongues were observed that are spiralling in opposite direction around the axon (see Fig.26).

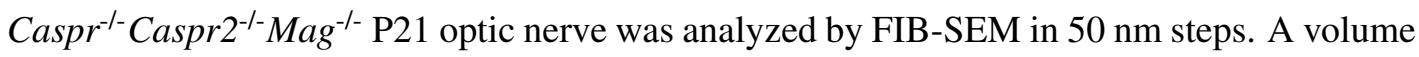
of $13 \mu \mathrm{m}$ x $6 \mu \mathrm{m}$ x $30 \mu \mathrm{m}$ of $\mathrm{Caspr}^{-{ }_{-}} \mathrm{Caspr}^{-{ }_{-}} \mathrm{Mag}^{-/-} \mathrm{P} 21$ optic nerve was analyzed by FIB-SEM in $50 \mathrm{~nm}$ steps. The 3D reconstruction of a split myelin sheath showed that both sheaths are independent from each other, indicating multiply myelination. 3D reconstruction of another split sheath event demonstrated that the inner tongue can invade in compacted myelin and continue to extent in there. The volume containend 26 myelinated axons. When followed through the stack, in 13 cases, the top myelin sheath opened up into a rosette of paranodal loop-like structures, indicating multiply myelinated axons. On 5 axons, the sheath could be followed, but the doubled layer ended in the inner tongue, indicating the inner tongue invading the compacted myelin.

3D reconstruction of myelinated sheaths from two different mutants affecting the paranodal junction complex provide evidence that Mag and the paranodal junction complex confine the 
a

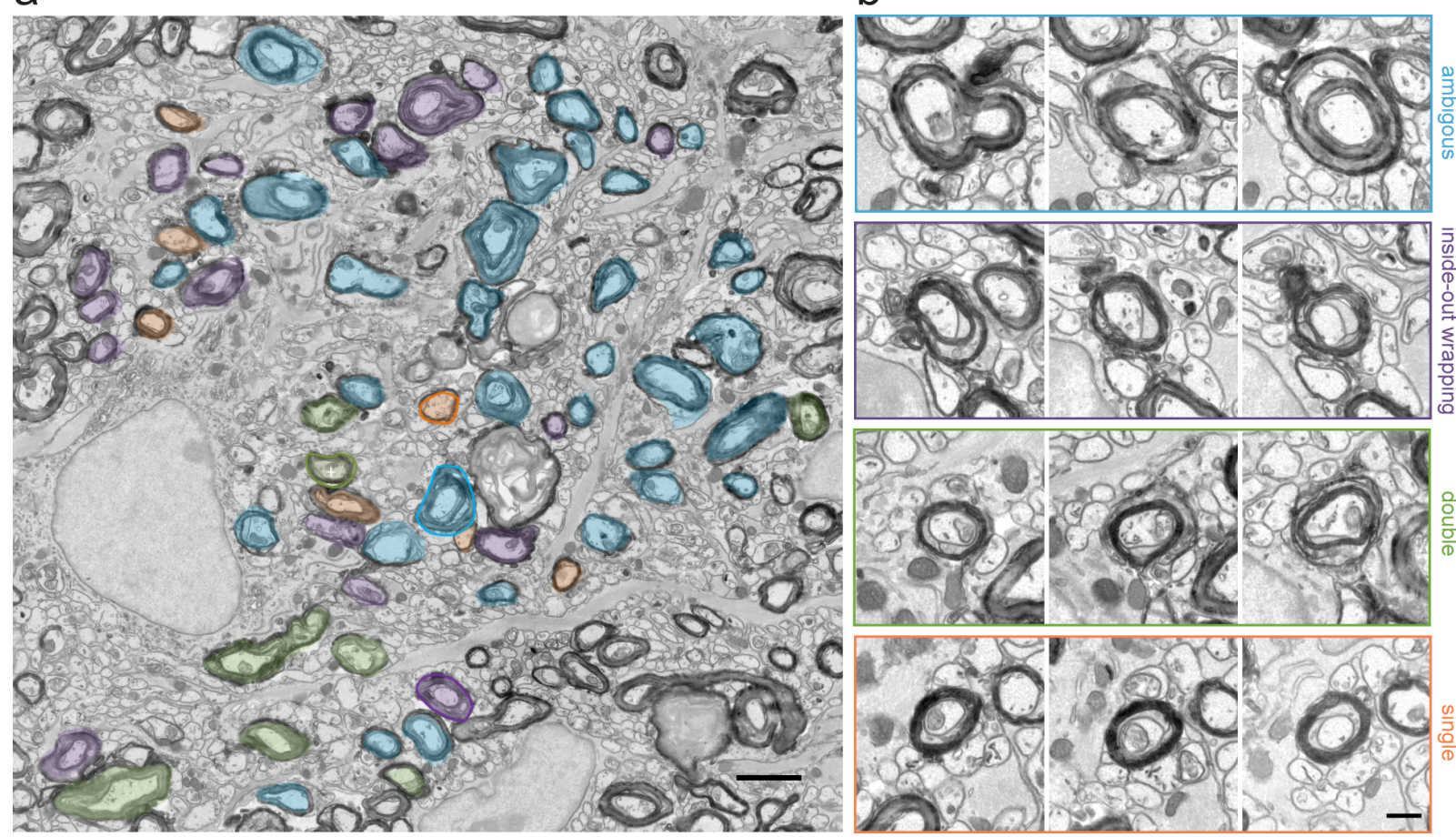

Figure 25: ATUM micrograph of a P15 $\mathrm{Cntn1}^{-/-} \mathrm{Mag}^{-/-}$double mutant optic nerve (a) Representative micrographs of one ATUM section (b) Representative images of myelinated axons that were followed through the volume, to categorize in: inside-out myelin, double myelin or ambiguous situation. Colors correspond to the respective categories, scale $b a r=2000 \mathrm{~nm}$. (Images were kindly provided by Dr. Martina Schifferer and Dr. Minou Djannatian) 


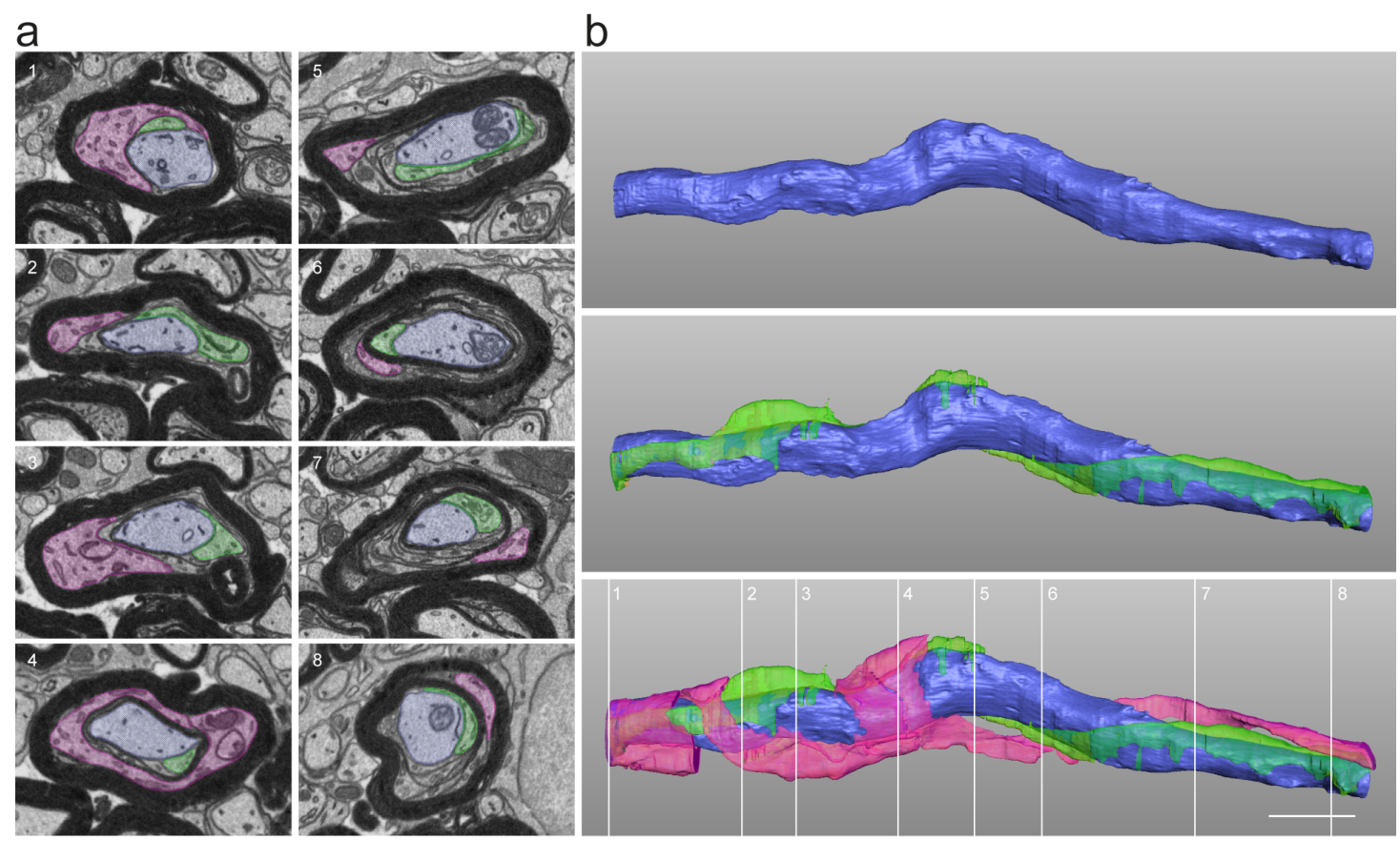

Figure 26: 3D FIB-SEM reconstruction of a P15 $\mathrm{CntnI}^{-/-} \mathbf{M a g}^{-/-}$double mutant optic nerve (a) Representative sections of the reconstructed axon (b) Reconstruction of an axon of the inside-out wrapping type, blue $=$ axon, green $=$ inner tongue, magenta $=$ secondary inner tonge, scale bar $=5 \mu \mathrm{m}$ (Images were kindly provided by $\mathrm{Dr}$. Martina Schifferer, Dr. Minou Djannatian and Manja Luckner). 
leading edge of the growing myelin sheath at its center, thereby guiding the wrapping and avoiding mistargeting to other structures. 

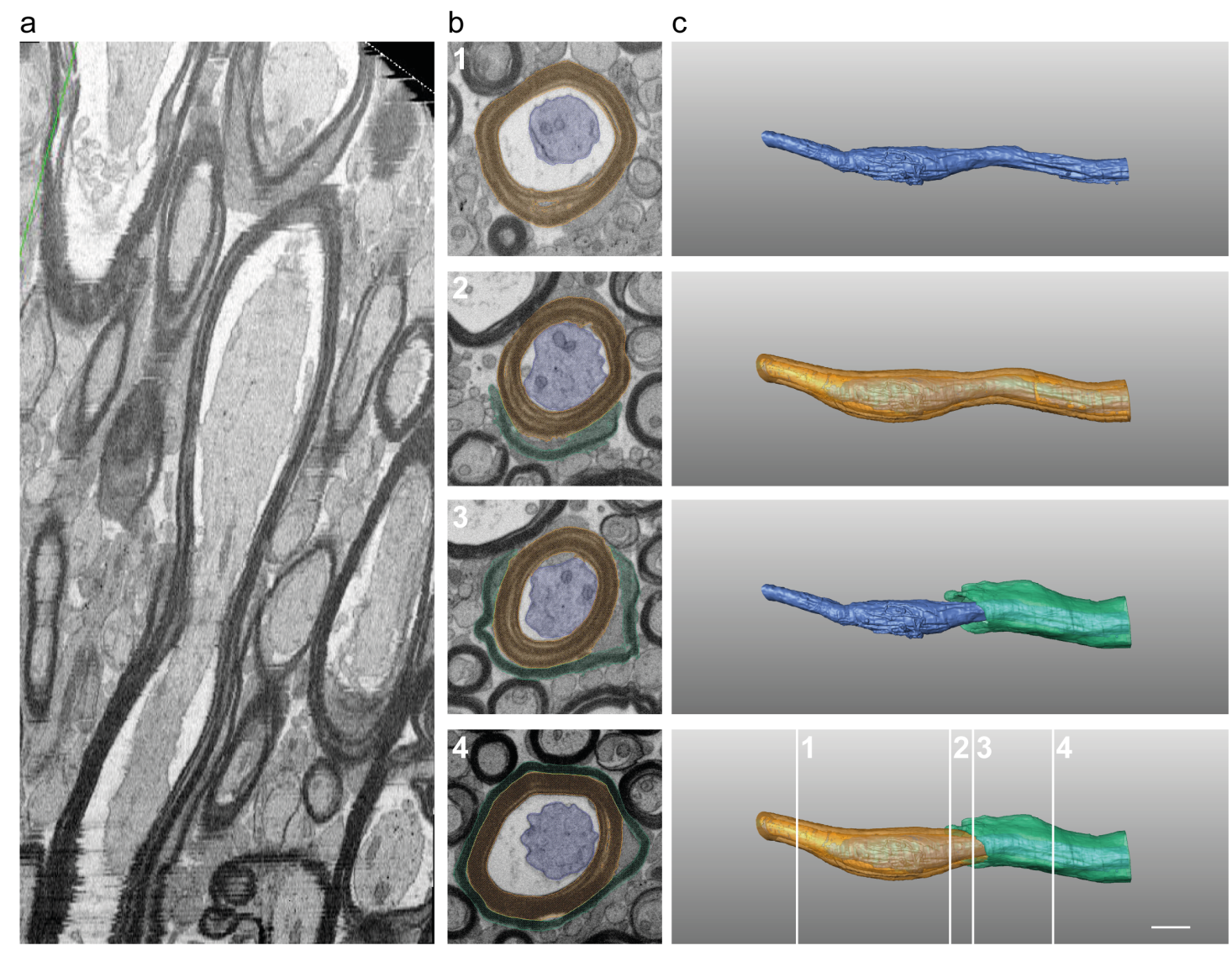

Figure 27: 3D FIB-SEM reconstruction of a P21 Cntnap $1^{-/-}$Cntnap $^{-/-} \mathbf{M a g}^{-/-}$double mutant optic nerve (a) Representative sections of the reconstructed axon (b) Reconstruction of an axon of the inside-out wrapping type, blue $=$ axon, green $=$ inner tongue, magenta $=$ secondary inner tongue, scale bar $=5 \mu \mathrm{m}$. 


\section{Discussion}

This study was designed to fuel the cellular model of myelin sheath formation with molecular mechanisms.

The formation of up to 80 myelin segments that grow from 20 to $200 \mu \mathrm{m}$ in lateral and up to 60 layers in width, oligodendrocytes have to coordinate a tremendous amount of membrane [62][63]. High caliber axons are preferentially myelinated whereas axons thinner than $0.2 \mu \mathrm{m}$ remain unwrapped. Axons in the range between 0.2 and $0.8 \mu \mathrm{m}$ that can either be myelinated or bare, hint to a regulatory mechanism for correct targeting [68]. The wrapping process was described recently: new membrane is added at the inner leaflet of the myelin sheath. The inner tongue is turning around the axon, adding new membrane layers from the inside. Compaction starts in the outer layers during lateral growth [82]. This model (see chapter 1.3.2 Fig.2) offers a detailed understanding of membrane movements and distribution but it lacks mechanistic information about involved molecules, in particular: cell surface adhesion molecules. Based on our observations, we developed a model that highlights the importance of close cell-cell contact through the paranodal junction adhesion complex together with the internodal adhesion of Mag. Our hypotheses are supported by results from various experiments in genetically modified zebrafish and mice including live and single frame 3D confocal imaging, 2D ultrastructural analysis by TEM and 3D ultrastructural analysis by FIB-SEM and ATUM.

In our model we propose, that neuron-oligodendrocyte adhesion is essential for lateral growth, precise wrapping and correct targeting of the myelin sheath. We reduced adhesive strength between neurons and oligodendrocytes by depleting inter-cellular adhesive molecules. Disruption of the paranodal adhesion complex (either by depletion of cntnapl, nfascb or cntnlb) leads to mistargeted cell body myelination in zebrafish but not in mice. The internodal adhesion protein mag itself is an important regulator of myelin sheath length in zebrafish, but when it is co-depleted together with cntnapl or cntnlb sheath growth is severely impaired. Double mutants of fish and mice exhibit hypomyelination and shortened sheaths. Nevertheless, overlapping myelin sheaths growing over nodes of Ranvier and on top of each other are often observed. Fur- 
thermore, double mutants exhibit another split-myelin form that we call 'inside-out wrapping'. The inner tongue is not confined at the periaxonal space but can invade compact myelin. Both myelin abnormalities were analyzed and reconstructed by $3 \mathrm{D}$ electron microscopy techniques.

\subsection{Localization of the paranodal junction complex}

The generation of a myelin sheath is a complex process between two interacting cells and can be structured in three steps: initiation, growth and termination. Two complementary mechanisms of node formation are well described, involving either paranodal adhesion or the extracellular matrix [105][110]. Proteins of the paranodal junction complex allow neuron-glia-interaction and seal it to the axonal cytoskeleton [173][93]. Node formation is the final step of sheath generation, but the involvement of adhesion molecules during initiation or growth phase stay elusive. Pedraza et al. demonstrated in cell culture experiments, that Caspr localizes spirally around the axon (reflecting the edges of single layers) and co-localizes with Nfasc155 [80]. We could confirm now by high-resolution expansion microscopy, that the spirally localization of Caspr seen in cell culture can also be observed in murine spinal cord sections (P21). Pedraza et al. concluded, that Caspr interacts between neuron and oligodendrocyte from initiation and follows the movement of the oligodendrocyte until it is localized nodally [80]. This hypothesis is supported by Nfasc isoform specific depletion studies. When Nfasc155 is lacking, the Caspr is diffusing along the axon not staying at the paranodes [174]. Myelination of the murine optic nerve starts at around P4 and is fully completed at P21. We tried to localize Caspr (the membrane spanning component of the paranodal junction complex) during different stages of the sheath development. Therefore, we co-immunostained spinal cord cryo-sections of P4, P7, P12 and P21 wildtype mice for Caspr and AnkG. The localization shift of AnkG signal from paranodal to nodal was used to determine the maturation state. We categorized 4 stages of node maturation and could confirm a transition from early to mature stages with development (P4 to P21), showing that AnkG localization is a suitable read out to assess nodal maturation state. Caspr immunostaining was able to visualize the paranodal localization, but no fluorescence signal was 
observed along the internode reflecting the tip of the inner tongue (as in vitro results suggested). We measured the size of the paranodal staining and the nodal gap. While the nodal gap is closing, the paranodal Caspr distribution stays unaltered. This raises the question if Caspr is really involved during early phases of sheath growth or if its main function is the termination and node formation. Cntnapl knock out studies in mice already showed that the number of myelin sheaths and the myelin thickness are unaltered indicating that initiation and growth are not affected [100]. Imaging in reporter fish lines, constantly expressing membrane-bound eGFP and transiently expressing caspr-YFP could demonstrate, that transport of caspr to the paranodes is evolutionary conserved in zebrafish, but an internodal localization was again absent. Since paranodal localization was shown for cntn1a-RFP and nfascb-mcherry constructs, we suggest that the mechanism forming the paranodal junction complex is similar in fish and mice. Thus, we could further identify nfascb and cntn $1 \mathrm{~b}$ as the equivalent orthologs of paranodal Cntn1 and Nfasc155 in mammals. It was shown before, that cntn1a is localized along axons and relocates to the nodes of Ranvier by myelin sheath growth [172].

\subsection{Paranodal junction proteins are required for correct myelin targeting}

The mechanisms of correct axon selection by oligodendrocytes are still debated. Despite neuronal activity and axonal caliber, other factors are needed to explain differentially myelinated axons or unmyelinated axons with caliber between 0.2 and $0.8 \mu \mathrm{m}$ [71]. Neuregulin-1 type III was identified as an instructive pro-myelinating signal in the PNS, but not the CNS [176][178]. The situation in the CNS seems to be more complex. The adhesion protein Necl1 was one of the first candidates for regulatory factors but showed only minor effects on myelin growth [73]. Redmond et al. followed a more promising path and identified Jam2 as a repellent agent on the membrane of neuronal cell bodies. The repulsive function was shown in PAX2-positive neurons of the dorsal horn, but it can not be considered as a CNS-wide universal mechanism [75]. Surprisingly, in zebrafish it seems to be sufficient to overproduce myelin to obtain myelination of neuronal cell bodies [76]. In the same study, Almeida et al. point out, that myelination of 
cell bodies can be seen in untreated wildtype fish during normal development that gets corrected later. We obtained the same results, showing that a basal level of cell body myelination in wildtype animals can be observed at $3 \mathrm{dpf}$. However, at $4 \mathrm{dpf}$ sheaths from myelinated cell bodies were almost completely retracted.

Balance of myelin production and available targets seems to be an additional but unspecific factor of myelin coordination. Therefore, it can be categorized together with neuronal activity or axonal caliber. Regulation by Jam2 is specific but locally limited. Neither Jam2 nor myelin/neuron balance can explain the whole process of axonal selection. It remains unclear why only cell bodies of certain neuronal subtypes are affected. Dendrites, microglia, oligodendrocytes, astrocytes or other cell types were not found to be myelinated in both models [75][76]. Structures as somatic synaptic terminals, different electro-statical configurations of the cell membranes or even other molecular cues could hinder myelination of other cells, but molecular guidance is still very likely. Since Necl1 was already looked at, we focused on the other major adhesion molecules present at the axon-glia interface: caspr, cntn1b and mag. Crispr/Cas9 mediated gene editing provided cntnap $^{-{ }^{--}}, \mathrm{cntnl}^{-{ }_{-}}$and $\mathrm{mag}^{-{ }^{--}}$mutant fish. In both paranodal mutants extensive cell body myelination was observed increasing with development pointing to a potential role in myelin targeting. Retraction from cell bodies was quantified and no difference

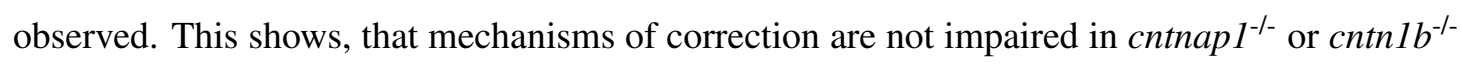
mutants and cell body myelination is the result of misguidance. $\mathrm{mag}^{-{ }^{-}-}$mutants were not distinguishable from wildtype fish by the number of myelinated cells bodies. Cell body myelination could not be detected in mutant mice. Co-immunostained cryo-sections (Mbp, NF and NeuN) of spinal cord and brain were analyzed, but myelin sheaths remained restricted to axons. It is very likely, that mammals developed additional mechanisms to guarantee myelin specificity (similar to Jam2) not present in fish. 


\subsection{Mag is regulating lateral growth in zebrafish}

Sheath length is greatly influencing the conduction velocity along an axon [89]. Myelin sheaths can have various lengths, mostly depending on the axonal caliber and their location in different CNS regions [17]. Peripheral nerves regulate the Schwann cell myelin properties by molecular signals like neuregulin-1 type III, but nothing similar was found in the CNS so far [176]. Oligodendrocytes extracted from cortex form shorter segments in vitro than oligodendrocytes of the spinal cord, suggesting an intrinsic length determination [177]. We analyzed the myelinated area in the spinal cord of our single mutant fish at $10 \mathrm{dpf}$. In contrast to paranodal mutants and wildtype controls, $\mathrm{mag}^{-/-}$mutants exhibited myelination reduced by $\sim 20 \%$. Mutants lacking the paranodal adhesion complex showed no difference in average sheath length, but $\mathrm{mag}^{-/-}$mutants showed significantly reduced segment lengths. More importantly, the average sheath length in mag $^{-/-}$mutants did not increase with development, as it did in paranodal mutants and wildtype controls. Shorter segments could be a reason for hypomyelination and delay of myelination observed in $\mathrm{Mag}^{-/-}$mice [122][124]. We observed hypomyelination in fish too, but the reduced lateral growth did not result in unmyelinated axons in between the sheaths, since shorter but normally formed segments filled in. A compensation by increased amounts of oligodendrocytes or sheaths per oligo were not observed.

\subsection{Paranodal proteins and Mag synergisticly coordinate lateral extension and correct wrapping}

We propose, that neuron-glia adhesion plays an important role in the wrapping process of the myelin sheath. Mutants lacking Mag, Caspr or Cntn1 alone, all form myelin in a relatively unaffected manner. Defects are mostly at the end of a sheath, affecting the formation of the nodes of Ranvier. The overall properties like extent of myelination, g-ratio and wrapping mechanisms are indistinguishable from wildtype animals. We found shorter sheaths and slight hypomyelination, in $\mathrm{mag}^{-/-}$fish. Zebrafish are suitable model organisms to follow in vivo myelination over a longer period of time [34][35]. Therefore, we decided to reduce neuron-glia adhesion to a greater ex- 
tent, by crossbreeding the heterozygous cntn $1 \mathrm{~b}^{+/-}$or cntnap $1^{+/-}$mutants with $\mathrm{mag}^{-{ }^{-/}}$animals to generate double mutants. Homozygous double mutant fish could be raised into adulthood, but were severely impaired and not able to breed. Imaging of the spinal cord at different devel-

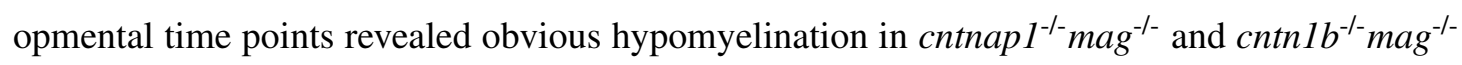
fish. Sheath lengths were reduced when depleting paranodal and internodal adhesion together. The extension and retraction rate of myelin sheaths were decreased by $\sim 50 \%$, indicating major changes in myelin organisation and wrapping.

Most surprisingly, many myelin sheaths exhibited regions with doubled intensity. This regions could be often identified as double myelin sheaths by transient co-expression of mbp:mcherry in some oligodendrocytes. Since eGFP-CAAX gets usually excluded from compacted sheaths, bright regions could in certain cases reflect wrapping and compaction defects. Hypomyelination would suggest that the remaining myelin sheaths try to distribute as much as possible to compensate the general myelin loss. This is not the case in the double mutants, in which sheaths were often stacked on the same axonal regions, leaving other parts unmyelinated. The process is progressive with development and the amount of myelin formed. Double myelinating sheaths are rare at $3 \mathrm{dpf}$ but at $10 \mathrm{dpf}$ many sheaths are located next to each other overgrowing and replacing the nodes of Ranvier. Since the nodes are essential for saltatory conduction, the implications for the nerve function are severe. Axons can compensate for the loss of 1-2 nodes (as was shown in early years of neuroscience by Tasaki et al. [37]) but when more nodes in a row are non-functional, conduction is blocked. Rearrangement of sodium channels along the axon to reestablish continuous conduction was observed in demyelinated peripheral axons, but can not occur under still existing myelin sheaths [179]. The neurological phenotype observed in double mutant fish may be a result of the conduction block, but the fact that they reach adulthood shows, that zebrafish have still enormous compensatory capabilities. Since the phenomenon of continuously myelinated axons is a special evolutionary feature of wildtype fish (the thick Mauthner axons) [32][33], it is possible, that some unknown Mauthner axon related mechanisms could rescue a residual nerve conduction. On the other hand, it is possible, that missing adhesion 
molecules along the Mauthner axons are the reason for their continuous myelination pattern.

Since reducing paranodal and internodal adhesion had tremendous consequences for the myelin sheaths in fish, we wanted to assess the membrane organization by an ultrastructural approach. By conventional fixation of zebrafish and electron microscopy, we could show that paranodal loop organisation is similar in wildtype fish as in mammals. Double mutants exhibited even

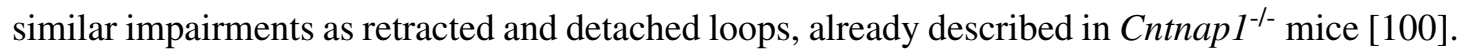

However, electron microscopy is dependent on the fixation method that can introduce artifacts and change the properties of the structure of interest [81]. Fixation techniques preserving the tissue close to the native state are mainly available in mice. Therefore, we decided to generate mouse models to analyze the myelin ultrastructure under the reduced adhesion condition. We crossbred mutant mice lacking Cntnapl or Cntnl with Mag knock out animals. Cntnap2 $2^{-/-}$animals were used to generate a triple Cntnap $1^{-C_{-}} \mathrm{Cntnap}^{-{ }^{--}} \mathrm{Mag}^{- \text {-- }^{-}}$mutant mouse with additionally reduced adhesion lacking the juxtaparanodal adhesion complex.

\subsubsection{Reducing paranodal and internodal adhesion in developing myelin by Cntn1 $^{-/-}$Mag $^{-/-}$depletion}

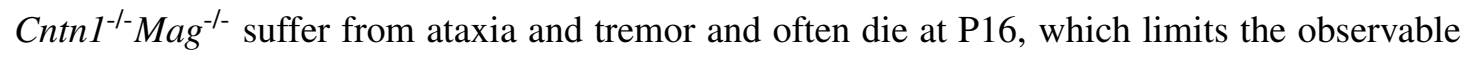
time points to developmental stages of myelin generation. Analyzed by conventional fixation

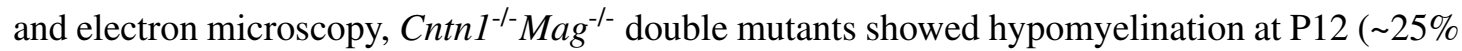
of wt) and P15 ( $50 \%$ of wt). The g-ratio in double mutants was slightly higher compared to wildtype controls and sheath lengths of double mutants (measured by immunohistochemistry) showed a $\sim 30 \%$ reduction of the average sheath length. Double mutant myelin showed many aberrant structures. We quantified myelin outfoldings (tomaculi), degenerated spots (whorls) and split-myelin. All of them were enriched in double mutants, indicating defects in myelin growth, compaction and survival. The increase in pathology implicates, that paranodal and internodal adhesion together are required for correct myelin sheath generation. Most of the myelin defects observed in zebrafish (doubled sheaths, reduced length) can be found in mice, except for 
myelinated cell bodies. It is very likely that neuronal cell bodies in mice are additionally protected by repellent molecules like Jam2 or incoming synaptic terminals [75]. Beside the reduced growth, the main observation in $\mathrm{CntnI}^{-\mathrm{I}_{-}} \mathrm{Mag}^{-/-}$mutants was the presence of split myelin. We followed the split myelin sheaths in 3D acquisitions and identified two different subtypes: doubled sheaths and a wrapping defect. Unfortunately, Cntn1 $1^{-/-} \mathrm{Mag}^{-/-}$can only be kept until P15. At this age, the optic nerve in wildtype animals is still being myelinated. To assess the situation in mature stages, we used Cntnap $1^{-/-}$mutants that exhibit a later onset of severe symptoms to generate Cntnap ${ }^{-{ }^{--}} \mathrm{Mag}^{-{ }^{--}}$animals. Mice with $\mathrm{Cntnap}^{-{ }^{--}}$mutations were introduced into the line to reduce adhesion even further.

\subsubsection{Reducing paranodal, juxtaparanodal and internodal adhesion in mature myelin by Cntnap $1^{-/-}$Cntnap2 $^{-/-}$Mag $^{-/-}$mutants}

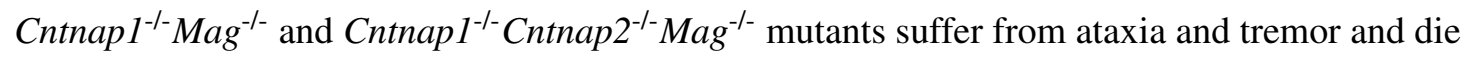
until P23, which enables us to observe later time points with matured myelination compared to Cntn1 mutants. It was possible to compare an early developmental stage (P12/13) and a timepoint when myelination in wildtype optic nerve is finished (P21/22) We fixed optic nerve conventionally for cross section analysis and by high-pressure freezing for longitudinal sections and 3D FIB-SEM. Cross section analysis revealed reduced myelination in Cntnap ${ }^{-{ }_{-1}} \mathrm{Mag}^{-{ }_{-}}$

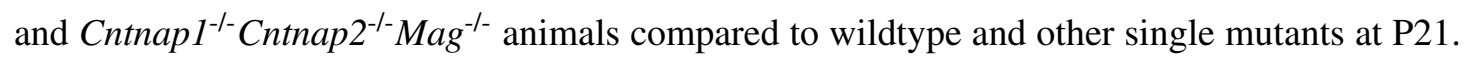
Interestingly, at P12 differences are not yet visible. Split myelinated sheaths could be identified in optic cross sections of both mutants lacking Cntnap1 and Mag. Quantification showed, that

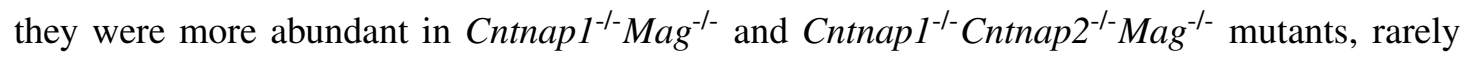
seen in single mutants and not at all in wildtype controls. Longitudinal sections revealed, when Cntnapl and Mag are missing, many sheaths are formed on top of each other as shown by multiple paranodal loop-like structures in one myelinated segment. In addition, we observed overgrown nodes and the absence of intact nodes of Ranvier. Interestingly, some heminodes appear quite normal whereas others are severely disordered. We propose, that paranodal loops 
can form without paranodal or internodal adhesion during growth, but adhesive force is too low for termination, resulting in disordered loops, misguided wrapping and overgrown sheaths. Another observation is, that paranodal loops can change their entire order. Usually the first loop next to a node is represented by the first wrap during sheath generation. The paranodal loops are ordered according to their time point of generation. The innermost loops are the youngest and are generated last. When all loops detach entirely, the inner tongue pushes the youngest loop close to the node, whereas the oldest loop is retracted. Cntnap2 depletion did not result in an additional effect. Since the Cntnap2/Cntn2Cntn2 complex is only present at the juxtaparanodes, it is possible, that Caspr2-mediated adhesion is weak and not able to compensate for the loss of paranodal and internodal adhesion. Depletion of Cntnap2 together with Mag did not aggravate the phenotype, suggesting that Caspr provides sufficient adhesive force to compensate for loss of juxtaparanodal and internodal adhesion.

\subsubsection{Two subtypes of split myelin are present in mutants lacking paranodal and internodal adhesion: doubled sheaths and "inside-out wrapping"}

By using 3D electron microscopy methods, we identified two different subtypes of split myelin: doubled sheaths and wrapping defects. The doubled sheaths could be explained by impaired termination or mistargeting. Kidd and Heath presented different ways of double myelin formation, based on observations in wildtype PNS nerves [180]. They propose, that neighboring sheaths overgrow the nodes of Ranvier and invade each others territorials. Another model describes, that non-neighbor processes recognize enlarged nodes of Ranvier as an unmyelinated axon, invade and push themselves under the sheaths next to the former node. According to Kidd and Heath, de novo myelination on top of existing myelin sheaths is very unlikely, but we can not exclude that by our observations. Beside from adhesion, as transmembrane proteins, Mag and Caspr would both be capable to act as receptors or ligands as was described before. Mag was shown to be able to influence axon calibers and mediate growth cone collapse in neurons [136][135]. Caspr, expressed in radial glia, can regulate growth of cortical neurons and astrocytes through 

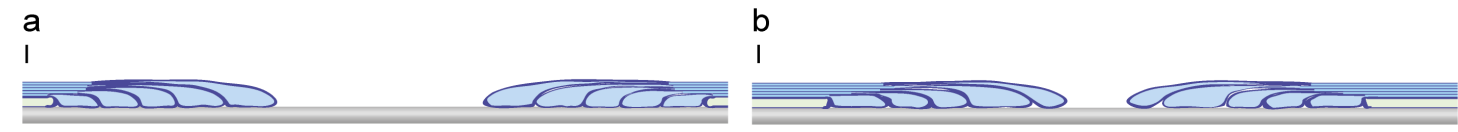

II

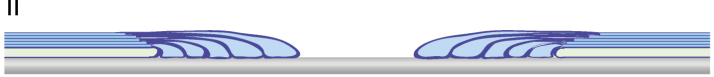

III

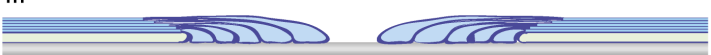

IV

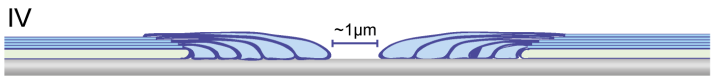

wildtype
II

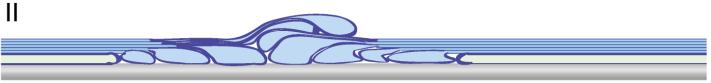

III

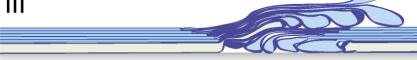

IV

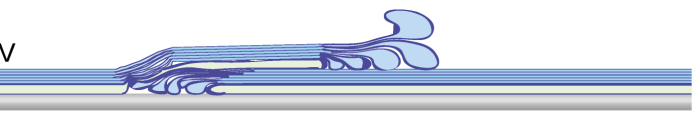

reduced adhesion

Figure 28: Model of overlapping myelin, presented in half cut view. (a) Model of wildtype node development (b) Model of overgrowing nodes and myelin double sheaths in mutants with reduced adhesion. One sheath is growing under its neighboring segment.

Notch-signalling [181].

Not all split myelin sheaths represent overgrown segments. In some cases split myelin could not be assigned to another independent sheath. We reconstructed myelin sheaths using 3D electron microscopy volumes and tracked those segments which resulted in a model that we call: "inside-out wrapping". We found, that when paranodal and internodal adhesion is reduced, the inner tongue can leave its confined position at the center of the myelinating sheath and grow into existing sheaths. We propose, that when paranodal loops are detached and retracted during sheath development, it is possible that the inner tongue does not stay close to the axon, but instead slips over the edge of a retracted layer, thereby wrapping over them in the opposite direction. Our model suggests that internodal adhesion by Mag with gangliosides and paranodal adhesion by Caspr/Cntn1/Nfasc155 together confine the inner tongue at the center of the myelin sheath. Since Mag and the paranodal adhesion complex work together, we wondered if the localization of Caspr is altered in Mag depleted animals. We assessed the localization of caspr 
in $\mathrm{mag}^{-/-}$fish by transient expression of caspr-YFP. No difference in position and size of the paranodes was observed. We stained for Caspr in optiv nerve cryo-sections of $\mathrm{Mag}^{-/-}$mice and obtained a similar result. Mag has no influence on the expression, localization and distribution of the paranodal adhesion proteins, indicated by Caspr. Therefore it is unlikely, that the synergistic cooperation of paranodal and internodal is based on direct molecular interaction between both structures. 
wildtype
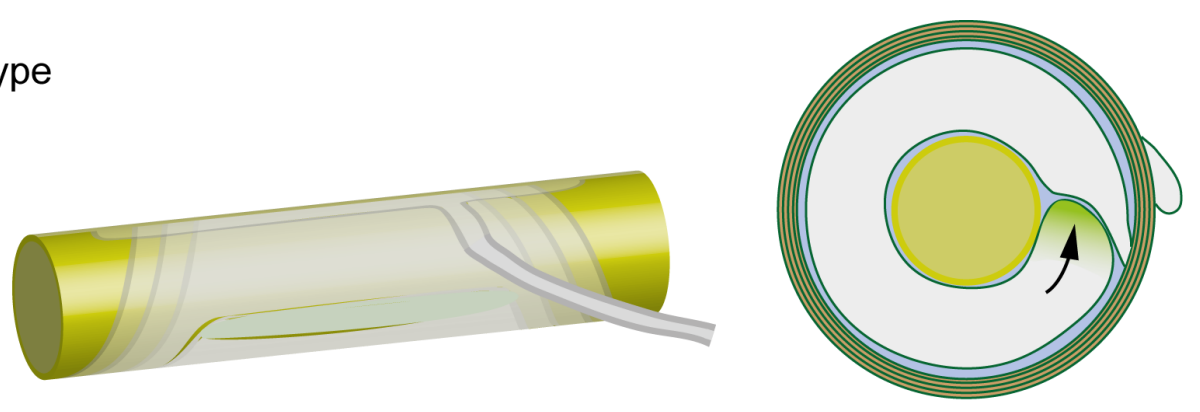

reduced adhesion

I
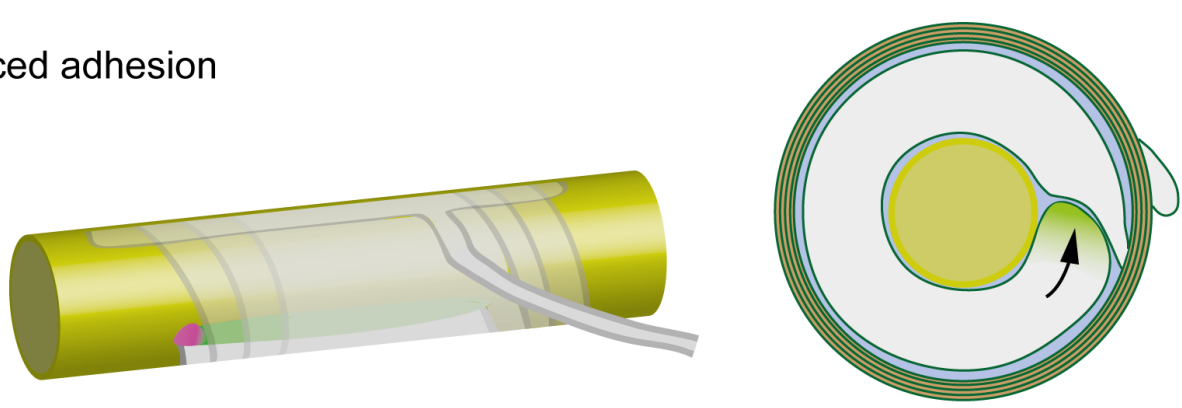

II
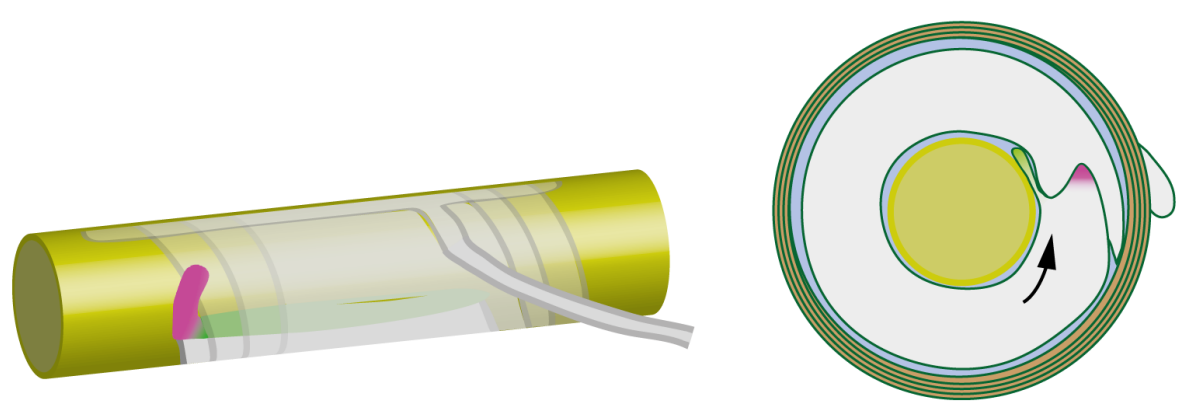

III
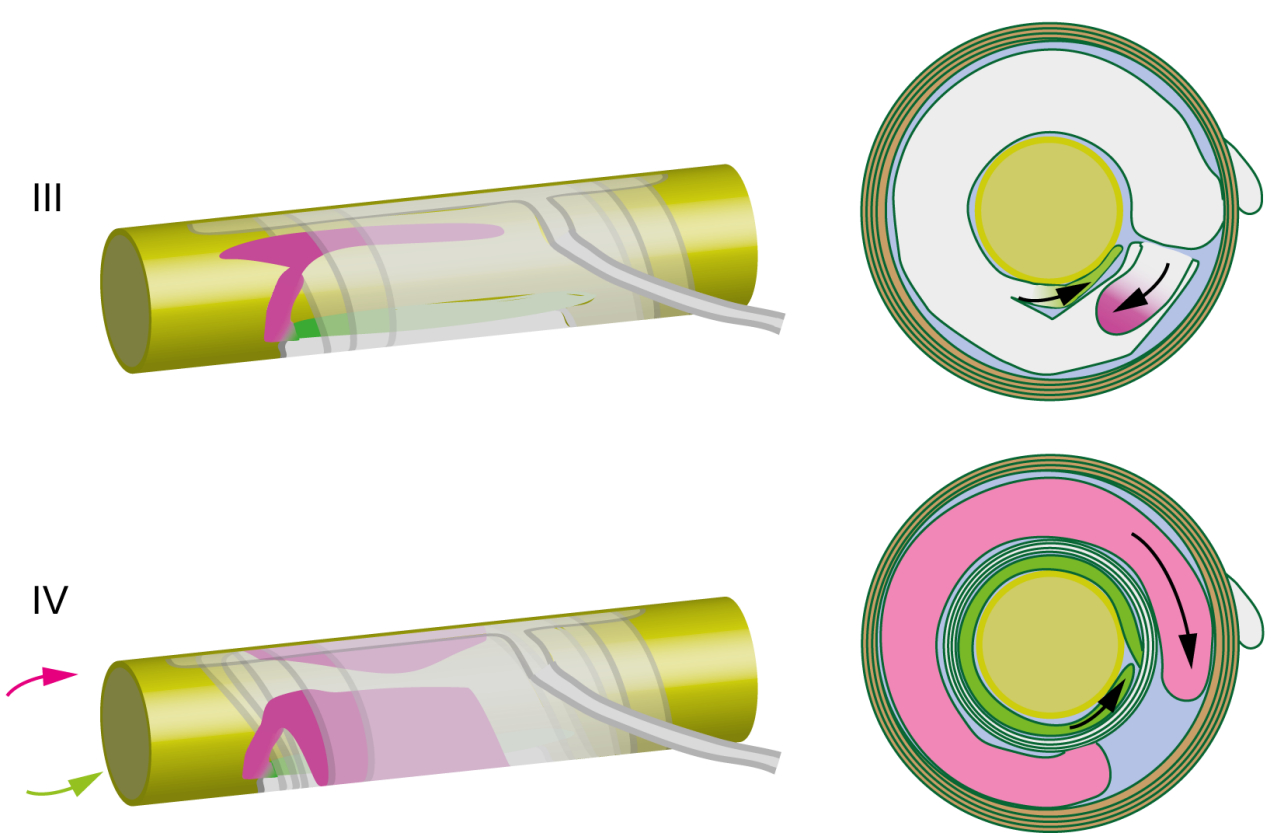

Figure 29: Model of "inside-out wrapping" in Cntnap1 ${ }^{-/-} \mathrm{Mag}^{-/-}$and $\mathrm{Cntn1}^{-/-} \mathrm{Mag}^{-/-}$myelin. In wildtype myelin sheaths, the active inner tongue adds layers of membrane from the inside. The first wrap becomes the outermost paranodal loop. [I] When axon-glia-adhesion is reduced, the layers can be retracted, releasing the inner tongue from the center. [II] The inner tongue is able to slip over the edge of a retracted layer. [III] The inner tongue is pushing in between two layers. [IV] The inner tongue is split. The inner tongue is actively myelinating at the inside, the second inner tongue extends into the myelin and wraps in the opposite direction. 


\section{Conclusion}

With this study, we extended the model of myelin generation by providing insights into the function of major adhesion molecules at the glial-axonal interface. We identified axon-oligodendroglial adhesion as a crucial factor for myelin sheath growth, wrapping and targeting.

Internodal adhesion is mainly generated by binding of the glial Mag binding to neuronal gangliosides. At paranodes, the paranodal junction complex, consisting of Caspr, Cntn1 and Nfasc155 generates a tight connection between axon and myelin sheath. We used Crispr/Cas9 mediated gene editing zebrafish reporter lines, to generate mutants with impaired paranodal and internodal adhesion. We disrupted the corresponding genes Cntnapl, Cntn1, Nfasc, Mag and generated combined mutants. Already existing mouse mutants were crossbred to obtain the same mutations in a higher model organism, more accessible to electron microscopy. Thus, we combined the strength of two model systems to obtain a comprehensive and dynamic model by exploiting time lapse confocal fluorescence imaging in zebrafish and state-of the art 3D electron microscopy in mouse tissue. Using live-imaging in fish, we could show that myelin growth was slowed down when internodal adhesion was reduced. When paranodal adhesion was reduced additionally, lateral growth was even more restricted. Double myelinated axons, myelinated cell bodies and distorted fluorescence patterns in myelinated sheaths could be found in zebrafish double mutants, indicating myelin mistargeting and defects.

In mice with reduced paranodal and internodal adhesion, we obtained similar results except of cell body myelination, which was only found in zebrafish. Myelin defects as tomaculi and whorls were more frequent. Split myelin sheaths were observed in 2D TEM optic nerve cross sections and followed by 3D-ATUM. We identified two subtypes of split myelin: true double myelin sheaths and the "inside-out-wrapping" type, exhibiting a misguided inner tongue moving in the opposite direction. Mutants of Cntnapl or Cntnl together with Mag exhibited no differences despite earlier onset of symptoms in $\mathrm{CntnI}^{-/-}$mutants, indicating that both are essential for the paranodal junction complex. With Caspr localization studies in $\mathrm{Mag}^{-/-}$mutants, showing no alteration, a molecular interaction of both adhesion systems is unlikely. 
By reducing axon-oligodendroglial adhesion to a minimum, we could show, that paranodal and internodal adhesion synergistically interact to enable lateral growth, to preserve correct guidance and to confine the inner tongue to the adaxonal center of the myelin sheath. Collectively, our results can explain how the two adhesive systems function in myelin growth and targeting. 


\section{References}

[1] Whitford MD, Freymiller GA, Clark RW. Avoiding the serpents tooth: predatorprey interactions between free-ranging sidewinder rattlesnakes and desert kangaroo rats. Anim Behav. 2017. 130, 7378.

[2] Macia S, Robinson MP, Craze P, Dalton R, Thomas JD. New observations on airborne jet propulsion (flight) in squid, with a review of previous reports. J Molluscan Stud. 2004. 70(May), 297299.

[3] Ponitz B, Schmitz A, Fischer D, Bleckmann H, Brücker C. Diving-flight aerodynamics of a peregrine falcon (Falco peregrinus). PLoS ONE. 2014. 9(2).

[4] Hodgkin AL. A note on conduction velocity. J Physiol. 1954. 125(1): 221224.

[5] Castelfranco AM, Hartline DK. The evolution of vertebrate and invertebrate myelin: a theoretical computational study. Journal of Computational Neuroscience. J Comput Neurosci. 2015. 38(3), 521538.

[6] Eaton RC. Neural mechanisms of startle behavior. Plenum Press. New York. 1984.

[7] WymanRJ, ThomasJB, SalkoffL,King DG. The Drosophila giant fiber system. In Neural Mechanisms of Startle Behavior. Eaton R ed. Plenum Press. New York. 1984. pp. 133161.

[8] Friedlaender B. Über die markhaltigen Nervenfasern und Neurochorde der Crustaceen und Anneliden. Mittheilungen aus der zoologischen Station Neapel. 1889. 9, 205265.

[9] Roots BI. The phylogeny of invertebrates and the evolution of myelin. Neuron Glia Biol. 2008. 4(2), 101109.

[10] Schweigreiter R, Roots BI, Bandtlow CE, Gould RM. Understanding Myelination Through Studying Its Evolution. Int Rev Neurobiol. 2006. 73(6), 219273.

[11] Davis AD, Weatherby TM, Hartline DK, Lenz PH. Myelin-like sheaths in copepod axons. Nature. 1999. 398(6728), 571.

[12] Kusano K. Electrical Activity and Structural Correlates of Giant Nerve Fibers in Kuruma Shrimp. J Cell Physiol. 1966. 68, 361384.

[13] Zalc B. The acquisition of myelin: An evolutionary perspective. Brain Res. 2016. 1641, 410. 
[14] Zalc B, Goujet D, Colman D. The origin of the myelination program in vertebrates. Curr Biol. 2008. 18(2005), 511512.

[15] Günther J. Impulse conduction in the myelinated giant fibers of the earthworm. Structure and function of the dorsal nodes in the median giant fiber. J Comp Neurol. 1976. 168(4), 505531.

[16] Nave KA. Myelination and support of axonal integrity by glia. Nature. 2010. 468, 244252.

[17] Hildebrand C, Remahl S, Persson H, Bjartmar C. Myelinated nerve fibres in the CNS. Prog Neurobiol. 1993. 40(3), 319384.

[18] Streisinger G, Walker C, Dower N, Knauber D, Singer F. Production of clones of homozygous diploid zebra fish (Brachydanio rerio). Nature. 1981. 291(5813), 293296.

[19] Hartline DK, Colman DR. Rapid Conduction and the Evolution of Giant Axons and Myelinated Fibers. Curr Biol. 2007. 17(1), 2935.

[20] Dooley K, Zon LI. Zebrafish: a model system for the study of human disease. Curr Opin Genet Dev. 2000. 10(3), 252256.

[21] Antinucci P, Hindges R. A crystal-clear zebrafish for in vivo imaging. Sci Rep. 2016. 6(June), 110.

[22] Kwan KM, Fujimoto E, Grabher C, Mangum BD, Hardy ME, Campbell DS, Parant JM, Yost HJ, Kanki JP, Chien C. The Tol2kit: A multisite gateway-based construction Kit for Tol2 transposon transgenesis constructs. Dev Dyn. 2007. 236(11), 30883099.

[23] Ablain J, Durand EM, Yang S, Zhou Y, Zon LI. A CRISPR/Cas9 Vector System for TissueSpecific Gene Disruption in Zebrafish. Dev Cell. 2015. pii: S1534-5807(15)00075-1.

[24] Glasauer SMK, Neuhauss SCF. Whole-genome duplication in teleost fishes and its evolutionary consequences. Mol Genet Genomics. 2014. 289(6), 10451060.

[25] Haenisch C, Diekmann H, Klinger M, Gennarini G, Kuwada JY, Stuermer CA. The neuronal growth and regeneration associated Cntnl (F3/F11/Contactin) gene is duplicated in fish: expression during development and retinal axon regeneration. Mol Cell Neurosci. 2005. Feb;28(2):361-74.

[26] Jeserich G, Klempahn K, Pfeiffer M. Features and functions of oligodendrocytes and myelin proteins of lower vertebrate species. J Mol Neurosci. 2008. 35, 117-126. 
[27] Yoshida M, Macklin WB. Oligodendrocyte development and myelination in GFPtransgenic zebrafish. J Neurosci Res. 2005. 81(1), 18.

[28] Buckley CE, Goldsmith P, Franklin RJM. Zebrafish myelination: a transparent model for remyelination? Dis Model Mech. 2008. 1, 221228.

[29] Sivron T, Cohen A, Duvdevani R, Jeserich G, Schwartz M. Glial response to axonal injury: In vitro manifestation and implication for regeneration. Glia. 1990. 3(4), 267276.

[30] Jeserich G, Stratmann A. In vitro differentiation of trout oligodendrocytes: evidence for an A2B5-positive origin. Brain Res Dev Brain Res. 1992. May 22;67(1):27-35.

[31] Celio MR. The Schmidt-Lantermann incisures of the myelin sheath of Mauther axons: site of longitudinal myelin growth. Brain Res. 1976. May 28;108(2):221-35.

[32] Greeff NG, Yasargil GM. Experimental evidence for saltatory propagation of the Mauthner axon impulse in the tench spinal cord. Brain Res. 1980. Jul 7;193(1):47-57.

[33] Hatta K, Korn H. Physiological properties of the Mauthner system in the adult zebrafish. J Comp Neurol. 1998. Jun 15;395(4):493-509.

[34] Wu W. Zebrafish: A new vertebrate model for visualizing myelination in vivo. Exp Neurol. 2014. 261, 278280.

[35] Brösamle C, Halpern ME. Characterization of myelination in the developing zebrafish. Glia. 2002. 39(1), 4757.

[36] Boullerne AI. The history of myelin. Exp Neurol. 2016. Sep;283(Pt B):431-45.

[37] Tasaki I. The electro-saltatory transmission of the nerve impulse and the effect of narcosis upon the nerve fiber. A J Physiol. 1939. 127, 211-227.

[38] Huxley AF, Stämpfli R. Evidence for saltatory conduction in peripheral myelinated nerve fibers. J Physiol. 1949. 108, 315-339.

[39] Geren BB, Raskind J. Development of the fine structure of the myelin sheath in sciatic nerves of chick embryos. Proc Natl Acad. Sci. 1953. 39, 880-884.

[40] Bunge MB, Bunge RP, Ris H. Ultrastructural study of remyelination in an experimental lesion in adult cat spinal cord. J Biophys Biochem Cytol. 1961. May;10:67-94.

[41] Bunge MB, Bunge RP, Pappas GD. Electron microscopic demonstration of connections between glia and myelin sheaths in the developing mammalian central nervous system. $\mathrm{J}$ Cell Biol. 1962. Feb;12:448-53. 
[42] Waxman S, Kocsis J, Stys P. The axon. Oxford Uni Press. 1995.

[43] Giedd JN, Blumenthal J, Jeffries NO, Castellanos FX, Liu H, Zijdenbos A, Paus T, Evans AC, Rapoport JL. Brain development during childhood and adolescence: a longitudinal MRI study. Nat Neurosci. 1999. Oct;2(10):861-3.

[44] Sowell ER, Peterson BS, Thompson PM, Welcome SE, Henkenius AL, Toga AW. Mapping cortical change across the human life span. Nat Neurosci. 2003. Mar;6(3):309-15.

[45] Auer F, Vagionitis S, Czopka T. Evidence for Myelin Sheath Remodeling in the CNS Revealed by In Vivo Imaging. Curr Biol. 2018. Feb 19;28(4):549-559.e3.

[46] Hill RA, Li AM, Grutzendler J. Lifelong cortical myelin plasticity and age-related degeneration in the live mammalian brain. Nat Neurosci. 2018. May;21(5):683-695.

[47] Mitew S, Hay CM, Peckham H, Xiao J, Koenning M, Emery B. Mechanisms regulating the development of oligodendrocytes and central nervous system myelin. Neuroscience. 2014. 276C, 2947.

[48] Glasser MF, Van Essen DC. Mapping human cortical areas in vivo based on myelin content as revealed by T1- and T2-weighted MRI. J Neurosci. 2011. Aug 10;31(32):11597616.

[49] de Hoz L, Simons M. The emerging functions of oligodendrocytes in regulating neuronal network behaviour. Bioessays. 2015. 37(1), 6069.

[50] Simons M, Lyons DA. Axonal selection and myelin sheath generation in the central nervous system. Curr Opin Cell Biol. 2013. 25: 5129.

[51] Zatorre RJ, Fields RD, Johansen-Berg H. Plasticity in gray and white: neuroimaging changes in brain structure during learning. Nat Neurosci. 2012. Mar 18;15(4):528-36.

[52] Liu J, Dietz K, DeLoyht JM, Pedre X, Kelkar D, Kaur J, Vialou V, Lobo MK, Dietz DM, Nestler EJ, Dupree J, Casaccia P. Impaired adult myelination in the prefrontal cortex of socially isolated mice. Nat Neurosci. 2012. 15: 16213.

[53] Schlegel AA, Rudelson JJ, Tse PU. White matter structure changes as adults learn a second language. J Cogn Neurosci. 2012. Aug;24(8):1664-70.

[54] Scholz J, Klein MC, Behrens TE, Johansen-Berg H. Training induces changes in whitematter architecture. Nat Neurosci. 2009. Nov;12(11):1370-1. 
[55] Pajevic S, Basser PJ, Fields RD. Role of myelin plasticity in oscillations and synchrony of neuronal activity. Neuroscience. 2014. Sep 12;276:135-47.

[56] Hercher C, Chopra V, Beasley CL. Evidence for morphological alterations in prefrontal white matter glia in schizophrenia and bipolar disorder. J Psychiatry Neurosci. 2014. 39(6):376-85.

[57] Miyata S, Taniguchi M, Koyama Y, Shimizu S, Tanaka T, Yasuno F, Tohyama M. Association between chronic stress-induced structural abnormalities in Ranvier nodes and reduced oligodendrocyte activity in major depression. Sci Rep. 2016. 6(February). 23084.

[58] Pan S, Chan JR. Regulation and dysregulation of axon infrastructure by myelinating glia. J Cell Biol. 2017. Dec 4;216(12):3903-3916.

[59] Pfeiffer SE, Warrington AE, Bansal R. The oligodendrocyte and its many cellular processes. Trends Cell Biol. 1993. 3, 191-197.

[60] Matthews MA, Duncan D. A quantitative study of morphological changes accompanying the initiation and progress of myelin production in the dorsal funiculus of the rat spinal cord. J Comp Neurol. 1971. May;142(1):1-22.

[61] Chong SY, Rosenberg SS, Fancy SP, Zhao C, Shen YA, Hahn AT, McGee AW, Xu X, Zheng B, Zhang LI, Rowitch DH, Franklin RJ, Lu QR, Chan JR. Neurite outgrowth inhibitor Nogo-A establishes spatial segregation and extent of oligodendrocyte myelination. Proc Natl Acad Sci. 2012. Jan 24;109(4):1299-304.

[62] Hildebrand C, Hahn R. Relation between myelin sheath thickness and axon size in spinal cord white matter of some vertebrate species. J Neurol Sci. 1978. Oct;38(3):421-34.

[63] Murray JA, Blakemore WF. The relationship between internodal length and fibre diameter in the spinal cord of the cat. J Neurol Sci. 1980. 45(1), 2941.

[64] Czopka T, Ffrench-Constant C, Lyons DA. Individual oligodendrocytes have only a few hours in which to generate new myelin sheaths in vivo. Dev Cell. 2013. Jun 24;25(6):599609.

[65] Demerens C, Stankoff B, Logak M, Anglade P, Allinquant B, Couraud F, Zalc B, Lubetzki C. Induction of myelination in the central nervous system by electrical activity. Proc Natl Acad Sci. 1996. Sep 3;93(18):9887-92. 
[66] Hamilton NB, Clarke LE, Arancibia-Carcamo IL, Kougioumtzidou E, Matthey M, Karadottir R, Whiteley L, Bergersen LH, Richardson WD, Attwell D. Endogenous GABA controls oligodendrocyte lineage cell number, myelination, and CNS internode length. Glia. 2016. 309321.

[67] Mensch S, Baraban M, Almeida R, Czopka T, Ausborn J, El Manira A, Lyons D. Synaptic vesicle release regulates myelin sheath number of individual oligodendrocytes in vivo. Nat Neurosci. 2015. 18(5), 610.

[68] Remahl S, Hildebrand C. Changing relation between onset of myelination and axon diameter range in developing feline white matter. J Neurol Sci. 1982. Apr;54(1):33-45.

[69] Rosenberg SS, Kelland EE, Tokar E, De la Torre AR, Chan JR. The geometric and spatial constraints of the microenvironment induce oligodendrocyte differentiation. Proc Natl Acad Sci. 2008. Sep 23;105(38):14662-7.

[70] Lee S, Leach MK, Redmond SA, Chong SY, Mellon SH, Tuck SJ, Feng ZQ, Corey JM, Chan JR. A culture system to study oligodendrocyte myelination processes using engineered nanofibers. Nat Methods. 2012. Sep;9(9):917-22.

[71] Tomassy GS, Berger DR, Chen HH, Kasthuri N, Hayworth KJ, Vercelli A, Seung HS, Lichtman JW, Arlotta P. Distinct profiles of myelin distribution along single axons of pyramidal neurons in the neocortex. Science. 2014. Apr 18;344(6181):319-24.

[72] Seidl AH, Rubel EW. Systematic and differential myelination of axon collaterals in the mammalian auditory brainstem. Glia. 2015. 487494.

[73] Park J, Liu B, Chen T, Li H, Hu X, Gao J, Zhu Y, Zhu Q, Qiang B, Yuan J, Peng X, Oiu M. Disruption of Nectin-like 1 cell adhesion molecule leads to delayed axonal myelination in the CNS. J Neurosci. 2008. 28(48), 128159.

[74] Zhu Y, Li H, Li K, Zhao X, An T, Hu X, Park J, Huang H, Bin Y, Qiang B, Yuan J, Peng X, Qiu M. Necl-4/SynCAM-4 is expressed in myelinating oligodendrocytes but not required for axonal myelination. PLoS One. 2013. May 20;8(5):e64264.

[75] Redmond SA, Mei F, Eshed-Eisenbach Y, Osso LA, Leshkowitz D, Shen YA, Kay JN, Aurrand-Lions M, Lyons DA, Peles E, Chan JR. Somatodendritic Expression of JAM2 Inhibits Oligodendrocyte Myelination. Neuron. 2016. 91(4), 824836.

[76] Almeida RG, Pan S, Cole KLH, Williamson JM, Early JJ, Czopka T, Klingseisen A, Chan JR, Lyons DA. Myelination of Neuronal Cell Bodies when Myelin Supply Exceeds Axonal Demand. Curr Biol. 2018. Apr 23;28(8):1296-1305.e5. 
[77] Bakhti M, Snaidero N, Schneider D, Aggarwal S, Möbius W, Janshoff A, Eckhardt M, Nave KA, Simons M. Loss of electrostatic cell-surface repulsion mediates myelin membrane adhesion and compaction in the central nervous system. Proc Natl Acad Sci. 2013. Feb 19;110(8):3143-8.

[78] Liu P, Du JL, He C. Developmental pruning of early-stage myelin segments during CNS myelination in vivo. Cell Res. 2013. 23(7), 962964.

[79] Sobottka B, Ziegler U, Kaech A, Becher B, Goebels N. CNS live imaging reveals a new mechanism of myelination: the liquid croissant model. Glia. 2011. Dec;59(12):1841-9.

[80] Pedraza L, Huang JK, Colman D. Disposition of axonal caspr with respect to glial cell membranes: Implications for the process of myelination. J Neurosci Res. 2009. Nov 15;87(15):3480-91.

[81] Möbius W, Cooper B, Kaufmann WA, Imig C, Ruhwedel T, Snaidero N, Saab AS, Varoqueaux F. Electron Microscopy of the Mouse Central Nervous System. Methods Cell Biol (Volume 96). 2010. Academic Press.

[82] Snaidero N, Möbius W, Czopka T, Hekking LH, Mathisen C, Verkleij D, Goebbels S, Edgar J, Merkler D, Lyons DA, Nave KA, Simons M. Myelin membrane wrapping of CNS axons by PI(3,4,5)P3-dependent polarized growth at the inner tongue. Cell. 2014. Jan 16;156(1-2):277-90.

[83] Velumian AA, Samoilova M, Fehlings MG. Visualization of cytoplasmic diffusion within living myelin sheaths of CNS white matter axons using microinjection of the fluorescent dye Lucifer Yellow. Neuroimage. 2011. May 1;56(1):27-34.

[84] Raasakka A, Ruskamo S, Kowal J, Barker R, Baumann A, Martel A, Tuusa J, Myllykoski M, Bürck J, Ulrich AS, Stahlberg H, Kursula P. Membrane Association Landscape of Myelin Basic Protein Portrays Formation of the Myelin Major Dense Line. Sci Rep. 2017. Jul 10;7(1):4974.

[85] Aggarwal S, Snaidero N, Pähler G, Frey S, Sánchez P, Zweckstetter M, Janshoff A, Schneider A, Weil MT, Schaap IA, Görlich D, Simons M. Myelin membrane assembly is driven by a phase transition of myelin basic proteins into a cohesive protein meshwork. PLoS Biol. 2013. 11(6):e1001577.

[86] Snaidero N, Velte C, Myllykoski M, Raasakka A, Ignatev A, Werner HB, Erwig MS, Möbius W, Kursula P, Nave KA, Simons M. Antagonistic Functions of MBP and CNP Establish Cytosolic Channels in CNS Myelin. Cell Rep. 2017. Jan 10;18(2):314-323. 
[87] Klugmann M, Schwab MH, Pühlhofer A, Schneider A, Zimmermann F, Griffiths IR, Nave KA. Assembly of CNS myelin in the absence of proteolipid protein. Neuron. 1997. Jan;18(1):59-70.

[88] Griffiths I, Klugmann M, Anderson T, Yool D, Thomson C, Schwab MH, Schneider A, Zimmermann F, McCulloch M, Nadon N, Nave KA. Axonal swellings and degeneration in mice lacking the major proteolipid of myelin. Science. 1998. Jun 5;280(5369):1610-3.

[89] Brill MH, Waxman SG, Moore JW, Joyner RW. Conduction velocity and spike configuration in myelinated fibres: computed dependence on internode distance. J Neurol Neurosurg Psychiatry. 1977. Aug;40(8):769-74.

[90] Pronker MF, Lemstra S, Snijder J, Heck AJR, Thies-Weesie DME, Pasterkamp RJ, Janssen BJC. Structural basis of myelin-associated glycoprotein adhesion and signalling. Nat Commun. 2016. 7(13584), 113.

[91] Rios JC, Melendez-Vasquez CV, Einheber S, Lustig M, Grumet M, Hemperly J, Peles E, Salzer JL. Contactin-associated protein (Caspr) and contactin form a complex that is targeted to the paranodal junctions during myelination. J Neurosci. 2000. Nov 15;20(22):8354-64.

[92] Gordon A, Adamsky K, Vainshtein A, Frechter S, Dupree JL, Rosenbluth J, Peles E. Caspr and caspr2 are required for both radial and longitudinal organization of myelinated axons. J Neurosci. 2014. Nov 5;34(45):14820-6.

[93] Brivio V, Faivre-Sarrailh C, Peles E, Sherman DL, Brophy PJ. Assembly of CNS Nodes of Ranvier in Myelinated Nerves Is Promoted by the Axon Cytoskeleton. Curr Biol. 2017. Apr 3;27(7):1068-1073.

[94] Narayanan SP, Flores AI, Wang F, Macklin WB. Akt signals through the mammalian target of rapamycin pathway to regulate CNS myelination. J Neurosci. 2009. May 27;29(21):6860-70.

[95] Flores AI, Narayanan SP, Morse EN, Shick HE, Yin X, Kidd G, Avila RL, Kirschner DA, Macklin WB. Constitutively active Akt induces enhanced myelination in the CNS. J Neurosci. 2008. Jul 9;28(28):7174-83.

[96] Goebbels S, Oltrogge JH, Kemper R, Heilmann I, Bormuth I, Wolfer S, Wichert SP, Möbius W, Liu X, Lappe-Siefke C, Rossner MJ, Groszer M, Suter U, Frahm J, 
Boretius S, Nave KA. Elevated phosphatidylinositol 3,4,5-trisphosphate in glia triggers cell-autonomous membrane wrapping and myelination. J Neurosci. 2010. Jun 30;30(26):8953-64.

[97] Goebbels S, Oltrogge JH, Wolfer S, Wieser GL, Nientiedt T, Pieper A, Ruhwedel T, Groszer M, Sereda MW, Nave KA. Genetic disruption of Pten in a novel mouse model of tomaculous neuropathy. EMBO Mol Med. 2012. Jun;4(6):486-99.

[98] Bartsch U, Montag D, Bartsch S, Schachner M. Multiply Myelinated Axons in the OpticNerve of Mice Deficient for the Myelin-Associated Glycoprotein. Glia. 1995. 14(2), 115122.

[99] Thaxton C, Pillai AM, Pribisko AL, Dupree JL, Bhat MA. Nodes of Ranvier act as barriers to restrict invasion of flanking paranodal domains in myelinated axons. Neuron. 2011. Jan 27;69(2):244-57.

[100] Bhat MA, Rios JC, Lu Y, Garcia-Fresco GP, Ching W, St Martin M, Li J, Einheber S, Chesler M, Rosenbluth J, Salzer JL, Bellen HJ. Axon-glia interactions and the domain organization of myelinated axons requires neurexin IV/Caspr/Paranodin. Neuron. 2001. May;30(2):369-83.

[101] Snaidero N, Simons M. Myelination at a glance. J Cell Sci. 2014. 127, 29993004.

[102] Erb M, Flueck B, Kern F, Erne B, Steck AJ, Schaeren-Wiemers N. Unraveling the differential expression of the two isoforms of myelin-associated glycoprotein in a mouse expressing GFP-tagged S-MAG specifically regulated and targeted into the different myelin compartments. Mol Cell Neurosci. 2006. Apr;31(4):613-27.

[103] Boyle ME, Berglund EO, Murai KK, Weber L, Peles E, Ranscht B. Contactin orchestrates assembly of the septate-like junctions at the paranode in myelinated peripheral nerve. Neuron. 2001. May;30(2):385-97.

[104] Pillai AM, Thaxton C, Pribisko AL, Cheng JG, Dupree JL, Bhat MA. Spatiotemporal ablation of myelinating glia-specific neurofascin (Nfasc NF155) in mice reveals gradual loss of paranodal axoglial junctions and concomitant disorganization of axonal domains. J Neurosci Res. 2009. Jun;87(8):1773-93.

[105] Zonta B, Tait S, Melrose S, Anderson H, Harroch S, Higginson J, Sherman DL, Brophy PJ. Glial and neuronal isoforms of Neurofascin have distinct roles in the assembly of nodes of Ranvier in the central nervous system. J Cell Biol. 2008. Jun 30;181(7):1169-77. 
[106] Gollan L, Sabanay H, Poliak S, Berglund EO, Ranscht B, Peles E. Retention of a cell adhesion complex at the paranodal junction requires the cytoplasmic region of Caspr. $\mathrm{J}$ Cell Biol. 2002. Jun 24;157(7):1247-56.

[107] Amor V, Zhang C, Vainshtein A, Zhang A, Zollinger DR, Eshed-Eisenbach Y, Brophy PJ, Rasband MN, Peles E. The paranodal cytoskeleton clusters Na+ channels at nodes of Ranvier. Elife. 2017. Jan 30;6. pii: e21392.

[108] Buttermore ED, Dupree JL, Cheng J, An X, Tessarollo L, Bhat MA. The cytoskeletal adaptor protein band $4.1 B$ is required for the maintenance of paranodal axoglial septate junctions in myelinated axons. J Neurosci. 2011. Jun 1;31(22):8013-24.

[109] Chang KJ, Zollinger DR, Susuki K, Sherman DL, Makara MA, Brophy PJ, Cooper EC, Bennett V, Mohler PJ, Rasband MN. Glial ankyrins facilitate paranodal axoglial junction assembly. Nat Neurosci. 2014. Dec;17(12):1673-81.

[110] Susuki K, Otani Y, Rasband MN. Submembranous cytoskeletons stabilize nodes of Ranvier. Exp Neurol. 2016. Sep;283(Pt B):446-51.

[111] Poliak S, Salomon D, Elhanany H, Sabanay H, Kiernan B, Pevny L, Stewart CL, Xu X, Chiu SY, Shrager P, Furley AJ, Peles E. Juxtaparanodal clustering of Shaker-like K+ channels in myelinated axons depends on Caspr2 and TAG-1. J Cell Biol. 2003. Sep 15;162(6):1149-60.

[112] Chen N, Koopmans F, Gordon A, Paliukhovich I, Klaassen RV, van der Schors RC, Peles $\mathrm{E}$, Verhage M, Smit AB, Li KW. Interaction proteomics of canonical Caspr2 (CNTNAP2) reveals the presence of two Caspr2 isoforms with overlapping interactomes. Biochim Biophys Acta. 2015. Jul;1854(7):827-33.

[113] Horresh I, Bar V, Kissil JL, Peles E. Organization of myelinated axons by Caspr and Caspr2 requires the cytoskeletal adapter protein 4.1B. J Neurosci. 2010. Feb 17;30(7):2480-9.

[114] Rodenas-Cuadrado P, Pietrafusa N, Francavilla T, La Neve A, Striano P, Vernes SC. Characterisation of CASPR2 deficiency disorder - a syndrome involving autism, epilepsy and language impairment. BMC Med Genet. 2016. Feb 3;17:8.

[115] Jahn O, Tenzer S, Werner HB. Myelin proteomics: molecular anatomy of an insulating sheath. Mol Neurobiol. 2009. Aug;40(1):55-72. 
[116] Quarles RH. Myelin-associated glycoprotein (MAG): Past, present and beyond. J Neurochem. 2007. 100(6), 14311448.

[117] Tropak MB, Johnson PW, Dunn RJ, Roder JC. Differential splicing of MAG transcripts during CNS and PNS development. Brain Res. 1988. Sep;464(2):143-55.

[118] Vinson M, Strijbos PJ, Rowles A, Facci L, Moore SE, Simmons DL, Walsh FS. Myelinassociated glycoprotein interacts with ganglioside GTlb. A mechanism for neurite outgrowth inhibition. J Biol Chem. 2001. 276, 20280-20285.

[119] Bartsch U, Kirchhoff F, Schachner M. Immunohistological localization of the adhesion molecules L1, N-CAM, and MAG in the developing and adult optic nerve of mice. J Comp Neurol. 1989. Jun 15;284(3):451-62.

[120] Trapp BD, Andrews SB, Cootauco C, Quarles R. The myelin-associated glycoprotein is enriched in multivesicular bodies and periaxonal membranes of actively myelinating oligodendrocytes. J Cell Biol. 1989. Nov;109(5):2417-26.

[121] Li C, Tropak MB, Gerlai R, Clapoff S, Abramow-Newerly W, Trapp B, Peterson A, Roder J. Myelination in the absence of myelin-associated glycoprotein. Nature. 1994. Jun 30;369(6483):747-50.

[122] Montag D, Giese KP, Bartsch U, Martini R, Lang Y, Blüthmann H, Karthigasan J, Kirschner DA, Wintergerst ES, Nave KA, Zielasek J, Toyka KV, Lipp HP, Schachner M. Mice deficient for the myelin-associated glycoprotein show subtle abnormalities in myelin. Neuron. 1994. Jul;13(1):229-46.

[123] Weiss MD, Hammer J, Quarles RH. Oligodendrocytes in aging mice lacking myelinassociated glycoprotein are dystrophic but not apoptotic. J Neurosci Res. 2000. 62: 772780.

[124] Bartsch S, Montag D, Schachner M, Bartsch U. Increased number of unmyelinated axons in optic nerves of adult mice deficient in the myelin-associated glycoprotein (MAG). Brain Res. 1997. 762(12), 231234.

[125] Biffiger K, Bartsch S, Montag D, Aguzzi A, Schachner M, Bartsch U. Severe hypomyelination of the murine CNS in the absence of myelin-associated glycoprotein and fyn tyrosine kinase. J Neurosci. 2000. 20(19), 74307437.

[126] Pan B, Fromholt SE, Hess EJ, Crawford TO, Griffin JW, Sheikh K, Schnaar RL. Myelinassociated glycoprotein and complementary axonal ligands, gangliosides, mediate axon 
stability in the CNS and PNS: neuropathology and behavioral deficits in single- and double-null mice. Exp Neurol. 2005. 195(1), 20817.

[127] Johnson PW, Abramow-Newerly W, Seilheimer B, Sadoul R, Tropak MB, Arquint M, Dunn RJ, Schachner M, Roder JC. Recombinant myelin-associated glycoprotein confers neural adhesion and neurite outgrowth function. Neuron. 1989. 3:377385.

[128] Marcus J, Dupree JL, Popko B. Myelin-associated glyco-protein and myelin galactolipids stabilize developing axo-glial interactions. J Cell Biol. 2002. 156, 567577.

[129] McKerracher L, David S, Jackson DL, Kottis V, Dunn RJ, Braun PE. Identification of myelin-associated glycoprotein as a major myelin-derived inhibitor of neurite growth. Neuron. 1994. Oct;13(4):805-11.

[130] Tang S, Shen YJ, DeBellard ME, Mukhopadhyay G, Salzer JL, Crocker PR, Filbin MT. Myelin-associated glycoprotein interacts with neurons via a sialic acid binding site at ARG118 and a distinct neurite inhibition site. J Cell Biol. 1997. Sep 22;138(6):1355-66.

[131] Al-Bashir N, Mellado W, Filbin MT. Sialic Acid Is Required for Neuronal Inhibition by Soluble MAG but not for Membrane Bound MAG. Front Mol Neurosci. 2016. Apr 1;9:21.

[132] Domeniconi M, Cao Z, Spencer T, Sivasankaran R, Wang K, Nikulina E, Kimura N, Cai H, Deng K, Gao Y, He Z, Filbin M. Myelin-associated glycoprotein interacts with the Nogo66 receptor to inhibit neurite outgrowth. Neuron. 2002. Jul 18;35(2):283-90.

[133] Venkatesh K, Chivatakarn O, Lee H, Joshi PS, Kantor DB, Newman BA, Mage R, Rader $\mathrm{C}$, Giger RJ. The Nogo-66 receptor homolog NgR2 is a sialic acid-dependent receptor selective for myelin-associated glycoprotein. J Neurosci. 2005. Jan 26;25(4):808-22.

[134] Atwal JK, Pinkston-Gosse J, Syken J, Stawicki S, Wu Y, Shatz C, Tessier-Lavigne M. PirB is a functional receptor for myelin inhibitors of axonal regeneration. Science. 2008. Nov 7;322(5903):967-70.

[135] Goh EL, Young JK, Kuwako K, Tessier-Lavigne M, He Z, Griffin JW, Ming GL. beta1integrin mediates myelin-associated glycoprotein signaling in neuronal growth cones. Mol Brain. 2008. Oct 15;1:10.

[136] Yin X, Crawford TO, Griffin JW, Tu P, Lee VM, Li C, Roder J, Trapp BD. Myelinassociated glycoprotein is a myelin signal that modulates the caliber of myelinated axons. J Neurosci. 1998. 18(6), 195362. 
[137] Umemori H, Sato S, Yagi T, Aizawa S, Yamamoto T. Initial events of myelination involve Fyn tyrosine kinase signalling. Nature. 1994. Feb 10;367(6463):572-6.

[138] Yamauchi J, Miyamoto Y, Torii T, Takashima S, Kondo K, Kawahara K, Nemoto N, Chan JR, Tsujimoto G, Tanoue A. Phosphorylation of cytohesin-1 by Fyn is required for initiation of myelination and the extent of myelination during development. Sci Signal. 2012. Sep 25;5(243):ra69.

[139] Fujita N, Kemper A, Dupree J, Nakayasu H, Bartsch U, Schachner M, Maeda N, Suzuki $\mathrm{K}$, Popko, B. The cytoplasmic domain of the large myelin-associated glycoprotein isoform is needed for proper CNS but not peripheral nervous system myelination. $\mathrm{J}$ Neurosci. 1998. 18(6), 19701978.

[140] Nguyen T, Mehta NR, Conant K, Kim KJ, Jones M, Calabresi PA, Melli G, Hoke A, Schnaar RL, Ming GL, Song H, Keswani SC, Griffin JW. Axonal protective effects of the myelin-associated glycoprotein. J Neurosci. 2009. Jan 21;29(3):630-7.

[141] Zeidan-Chulia F, de Oliveira BN, Casanova MF, Casanova EL, Noda M, Salmina AB, Verkhratsky A. Up-Regulation of Oligodendrocyte Lineage Markers in the Cerebellum of Autistic Patients: Evidence from Network Analysis of Gene Expression. Mol Neurobiol. 2016. Aug;53(6):4019-4025.

[142] Felsky D, Voineskos AN, Lerch JP, Nazeri A, Shaikh SA, Rajji TK, Mulsant BH, Kennedy JL. Myelin-associated glycoprotein gene and brain morphometry in schizophrenia. Front Psychiatry. 2012. May 3;3:40.

[143] Novarino G, Fenstermaker AG, Zaki MS, Hofree M, Silhavy JL, Heiberg AD, Abdellateef M, Rosti B, Scott E, Mansour L, Masri A, Kayserili H, Al-Aama JY, Abdel-Salam GMH, Karminejad A, Kara M, Kara B, Bozorgmehri B, Ben-Omran T, Mojahedi F, El Din Mahmoud IG, Bouslam N, Bouhouche A, Benomar A, Hanein S, Raymond L, Forlani S, Mascaro M, Selim L, Shehata N, Al-Allawi N, Bindu PS, Azam M, Gunel M, Caglayan A, Bilguvar K, Tolun A, Issa MY, Schroth J, Spencer EG, Rosti RO, Akizu N, Vaux KK, Johansen A, Koh AA, Megahed H, Durr A, Brice A, Stevanin G, Gabriel $\mathrm{SB}$, Ideker T, Gleeson JG. Exome sequencing links corticospinal motor neuron disease to common neurodegenerative disorders. Science. 2014. Jan 31;343(6170):506-511.

[144] Lossos A, Elazar N, Lerer I, Schueler-Furman O, Fellig Y, Glick B, Zimmerman BE, Azulay H, Dotan S, Goldberg S, Gomori JM, Ponger P, Newman JP, Marreed H, Steck AJ, Schaeren-Wiemers N, Mor N, Harel M, Geiger T, Eshed-Eisenbach Y, Meiner V, Peles E. 
Myelin-associated glycoprotein gene mutation causes Pelizaeus-Merzbacher disease-like disorder. Brain. 2015. Sep;138(Pt 9):2521-36.

[145] Poltorak M, Steck AJ, Schachner M. Reactivity with neural cell adhesion molecules in sera from patients with demyelinating diseases. Neurosci Lett. 1986. Apr 11;65(2):199203.

[146] Wajgt A, Górny M. CSF antibodies to myelin basic protein and to myelin-associated glycoprotein in multiple sclerosis. Evidence of the intrathecal production of antibodies. Acta Neurol Scand. 1983. Nov;68(5):337-43.

[147] Buser JR, Maire J, Riddle A, Gong X, Nguyen T, Nelson K, Luo NL, Ren J, Struve J, Sherman LS, Miller SP, Chau V, Hendson G, Ballabh P, Grafe MR, Back SA. Arrested preoligodendrocyte maturation contributes to myelination failure in premature infants. Ann Neurol. 2012. 71(1):93-109.

[148] Bartzokis G. Alzheimer's disease as homeostatic responses to age-related myelin breakdown. Neurobiol Aging. 2011. 32(8), 13411371.

[149] Fennema-Notestine C, Archibald SL, Jacobson MW, Corey-Bloom J, Paulsen JS, Peavy GM, Gamst AC, Hamilton JM, Salmon DP, Jernigan TL. In vivo evidence of cerebellar atrophy and cerebral white matter loss in huntington disease. Neurology. 2004. 63, 989995.

[150] Bonkowsky J, Nelson C, Kingston J, Filloux F, Mundorff M, Srivastava R. The burden of inherited leukodystrophies in children. Neurology. 2010. 75(8):718-725.

[151] Conant A, Curiel J, Pizzino A, Sabetrasekh P, Murphy J, Bloom M, Evans SH, Helman B, Taft RJ, Simons C, Whitehead MT, Moore SA, Vanderver A. Absence of Axoglial Paranodal Junctions in a Child With CNTNAP1 Mutations, Hypomyelination, and Arthrogryposis. J Child Neurol. 2018. 1-9.

[152] Low K, Stals K, Caswell R, Wakeling M, Clayton-Smith J, Donaldson A, Foulds N, Norman A, Splitt M, Urankar K, Vijayakumar K, Majumdar A, Study DDD, Ellard S, Smithson S. Phenotype of CNTNAP1: a study of patients demonstrating a specific severe congenital hypomyelinating neuropathy with survival beyond infancy. Eur J Hum Genet. 2018.

[153] Lakhani S, Doan R, Almureikhi M, Partlow JN, Al Saffar M, Elsaid MF, Alaaraj N, James Barkovich A, Walsh CA, Ben-Omran T. Identification of a novel CNTNAP1 mutation 
causing arthrogryposis multiplex congenita with cerebral and cerebellar atrophy. Eur J Med Genet. 2017. May;60(5):245-249.

[154] Compton AG, Albrecht DE, Seto JT, Cooper ST, Ilkovski B, Jones KJ, Challis D, Mowat D, Ranscht B, Bahlo M, Froehner SC, North KN. Mutations in contactin-1, a neural adhesion and neuromuscular junction protein, cause a familial form of lethal congenital myopathy. Am J Hum Genet. 2008. Dec;83(6):714-24.

[155] Anazi S, Maddirevula S, Salpietro V, Asi YT, Alsahli S, Alhashem A, Shamseldin HE, AlZahrani F, Patel N, Ibrahim N, Abdulwahab FM, Hashem M, Alhashmi N, Al Murshedi F, Al Kindy A, Alshaer A, Rumayyan A, Al Tala S, Kurdi W, Alsaman A, Alasmari A, Banu S, Sultan T, Saleh MM, Alkuraya H, Salih MA, Aldhalaan H, Ben-Omran T, Al Musafri F, Ali R, Suleiman J, Tabarki B, El-Hattab AW, Bupp C, Alfadhel M, Al Tassan N, Monies D, Arold ST, Abouelhoda M, Lashley T, Houlden H, Faqeih E, Alkuraya FS. Expanding the genetic heterogeneity of intellectual disability. Hum Genet. 2017. Nov;136(11-12):1419-1429.

[156] Nawaz S, Sánchez P, Bodensiek K, Li S, Simons M, Schaap IA. Cell visco-elasticity measured with AFM and optical trapping at sub-micrometer deformations. PLoS One. 2012. 7(9):e45297.

[157] Derfuss T, Meinl E. Identifying autoantigens in demyelinating diseases: valuable clues to diagnosis and treatment? Curr Opin Neurol. 2012. Jun;25(3):231-8.

[158] Mathey EK, Derfuss T, Storch MK, Williams KR, Hales K, Woolley DR, Al-Hayani A, Davies SN, Rasband MN, Olsson T, Moldenhauer A, Velhin S, Hohlfeld R, Meinl E, Linington C. Neurofascin as a novel target for autoantibody-mediated axonal injury. J Exp Med. 2007. Oct 1;204(10):2363-72.

[159] Schindelin J, Arganda-Carreras I, Frise E. Fiji: an open-source platform for biologicalimage analysis. Nat Methods. 2012. 9(7): 676-682.

[160] Schneider CA, Rasband WS, Eliceiri KW. NIH Image to ImageJ: 25 years of image analysis. Nat Methods. 2012. 9(7): 671-675.

[161] Rueden CT, Schindelin J, Hiner MC. ImageJ2: ImageJ for the next generation of scientific image data. BMC Bioinformatics. 2017. 18:529.

[162] Belevich I, Joensuu M, Kumar D, Vihinen H, Jokitalo E. Microscopy Image Browser: A platform for segmentation and analysis of multidimensional datasets. PLoS Biology. 2016. Jan 4;14(1):e1002340. 
[163] R Development Core Team. $R$ : A language and environment for statistical computing. $\mathrm{R}$ Foundation for Statistical Computing, Vienna, Austria. 2008. ISBN 3-900051-07-0.

[164] Labun K, Montague TG, Gagnon JA, Thyme SB, Valen E. CHOPCHOP v2: a web tool for the next generation of CRISPR genome engineering. Nucleic Acids Res. 2016. Jul 8;44(W1):W272-6.

[165] Montague TG, Cruz JM, Gagnon JA, Church GM, Valen E. CHОРСНОР: $a$ CRISPR/Cas9 and TALEN web tool for genome editing. Nucleic Acids Res. 2014. Jul;42:W401-7.

[166] Chen F, Tillberg PW, Boyden ES. Optical imaging. Expansion microscopy. Science. 2015. Jan 30;347(6221):543-8.

[167] Peters PJ, Pierson J. Immunogold labeling of thawed cryosections. Methods Cell Biol. 2008. 88:131-49.

[168] Almeida RG, Czopka T, Ffrench-Constant C, Lyons DA. Individual axons regulate the myelinating potential of single oligodendrocytes in vivo. Development. 2011. Oct;138(20):4443-50.

[169] Tapia JC, Wylie JD, Kasthuri N, Hayworth KJ, Schalek R, Berger DR, Guatimosim C, Seung HS, Lichtman JW. Pervasive synaptic branch removal in the mammalian neuromuscular system at birth. Neuron. 2012. Jun 7;74(5):816-29.

[170] Kasthuri N, Hayworth KJ, Berger DR, Schalek RL, Conchello JA, Knowles-Barley S, Lee D, Vázquez-Reina A, Kaynig V, Jones TR, Roberts M, Morgan JL, Tapia JC, Seung HS, Roncal WG, Vogelstein JT, Burns R, Sussman DL, Priebe CE, Pfister H, Lichtman JW. Saturated Reconstruction of a Volume of Neocortex. Cell. 2015. Jul 30;162(3):648-61.

[171] Cardona A, Saalfeld S, Schindelin J, Arganda-Carreras I, Preibisch S, Longair M, Tomancak P, Hartenstein V, Douglas RJ. TrakEM2 software for neural circuit reconstruction. PLoS One. 2012. 7(6):e38011.

[172] Koudelka S, Voas MG, Almeida RG, Baraban M, Soetaert J, Meyer MP, Talbot WS, Lyons DA. Individual Neuronal Subtypes Exhibit Diversity in CNS Myelination Mediated by Synaptic Vesicle Release. Curr Biol. 2016. Jun 6;26(11):1447-55.

[173] Ho TS, Zollinger DR, Chang KJ, Xu M, Cooper EC, Stankewich MC, Bennett V, Rasband MN. A hierarchy of ankyrin-spectrin complexes clusters sodium channels at nodes of Ranvier. Nat Neurosci. 2014. Dec;17(12):1664-72. 
[174] Taylor AM, Saifetiarova J, Bhat MA. Postnatal Loss of Neuronal and Glial Neurofascins Differentially Affects Node of Ranvier Maintenance and Myelinated Axon Function. Front Cell Neurosci. 2017. Feb 3;11:11.

[175] Saifetiarova J, Liu X, Taylor AM, Li J, Bhat MA. Axonal domain disorganization in Caspr1 and Caspr2 mutant myelinated axons affects neuromuscular junction integrity, leading to muscle atrophy. J Neurosci Res. 2017. 95(7):1373-1390.

[176] Michailov GV, Sereda MW, Brinkmann BG, Fischer TM, Haug B, Birchmeier C, Role L, Lai C, Schwab MH, Nave KA. Axonal neuregulin-1 regulates myelin sheath thickness. Science. 2004. Apr 30;304(5671):700-3.

[177] Bechler ME, Byrne L, Ffrench-Constant C. CNS Myelin Sheath Lengths Are an Intrinsic Property of Oligodendrocytes. Curr Biol. 2015. Sep 21;25(18):2411-6.

[178] Taveggia C, Zanazzi G, Petrylak A, Yano H, Rosenbluth J, Einheber S, Xu X, Esper RM, Loeb JA, Shrager P, Chao MV, Falls DL, Role L, Salzer JL. Neuregulin-1 type III determines the ensheathment fate of axons. Neuron. 2005. Sep 1;47(5):681-94.

[179] Foster RE, Whalen CC, Waxman SG. Reorganization of the axon membrane in demyelinated peripheral nerve fibers: morphological evidence. Science. 1980. Nov 7;210(4470):661-3.

[180] Kidd GJ, Heath JW. Double myelination of axons in the sympathetic nervous system of the mouse. II. Mechanisms of formation. J Neurocytol. 1988. 17(2), 263276.

[181] Wu ZQ, Li D, Huang Y, Chen XP, Huang W, Liu CF, Zhao HQ, Xu RX, Cheng M, Schachner M, Ma QH. Caspr Controls the Temporal Specification of Neural Progenitor Cells through Notch Signaling in the Developing Mouse Cerebral Cortex. Cereb Cortex. 2017. Feb 1;27(2):1369-1385. 


\section{Appendix}

\section{Knock out validation by qRT-PCR}
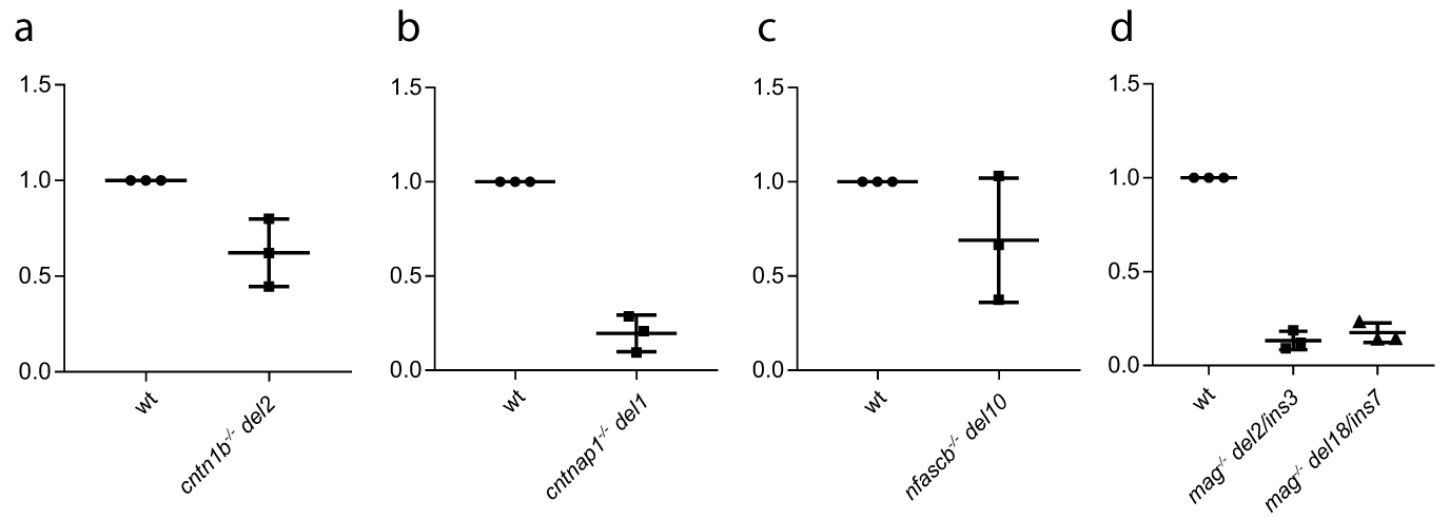

Figure 30: Knock out validation by mRNA levels in Crispr/Cas9 edited fish, measured by qRT-PCR. (a) $c n t n 1 b^{-/-}$(b) cntnapl $^{-I_{-}}$(c) $n f a s c b^{-{ }^{-}}$, (d) $\mathrm{mag}^{-{ }^{-}}$One mutation was tested for cntnlb, cntnapl, nfascb and two for mag. mRNA levels of cntnlb and $n f a s c b$ were reduced by about $40 \%$, mag and cntnapl mutants showed reduction by about $85 \%$. 


\section{Localization of paranodal proteins in zebrafish}

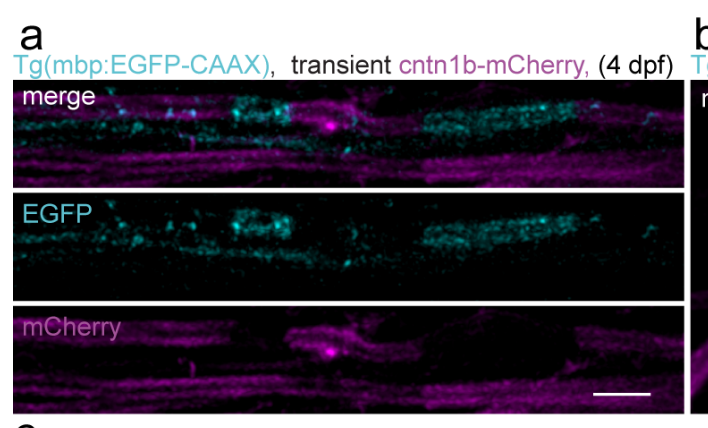

C
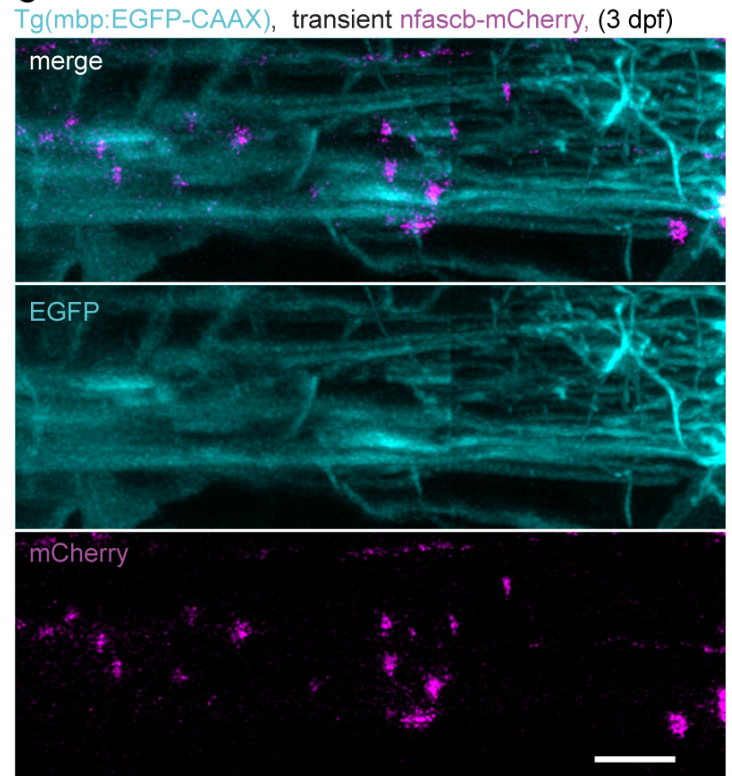

b

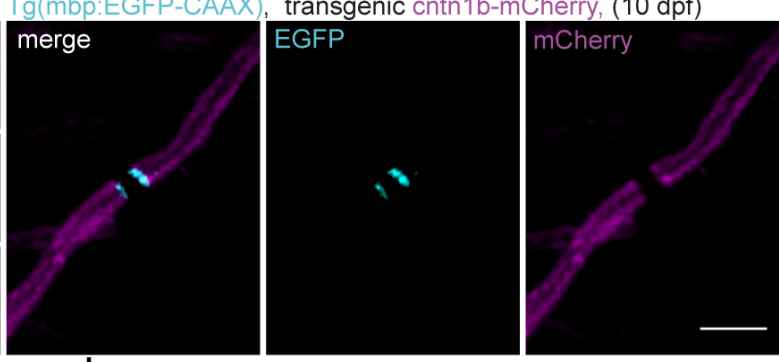

d

Tg(mbp:EGFP-CAAX), transgenic caspr-YFP, (3 dpf)

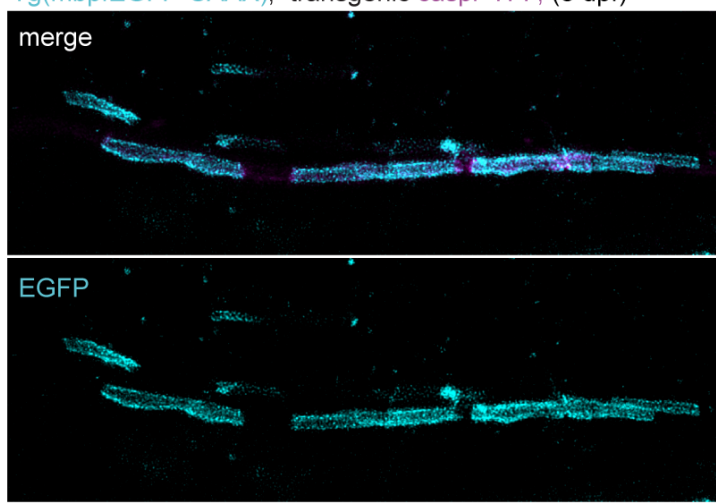

mCherry

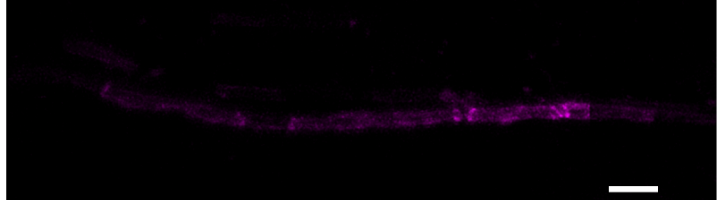

Figure 31: Confocal micrographs of the localization of paranodal proteins using fusion proteins (a) cntn1b-mcherry at $4 \mathrm{dpf}$, scale bar $=3 \mu \mathrm{m}$ (b) cntn1b-mcherry at 10 dpf, scale bar $=3 \mu \mathrm{m}$ (c) nfascb-mcherry at $3 \mathrm{dpf}$, scale bar $=5 \mu \mathrm{m}$ (d) caspr-YFP at $3 \mathrm{dpf}$, scale bar $=5 \mu \mathrm{m}$. 